JOURNAL OF THE

AMERICAN MATHEMATICAL SOCIETY

Volume 12, Number 2, April 1999, Pages 335-380

S $0894-0347$ (99)00295-7

\title{
DOUBLE BRUHAT CELLS AND TOTAL POSITIVITY
}

\author{
SERGEY FOMIN AND ANDREI ZELEVINSKY
}

\section{Contents}

0. Introduction 336

1. Main results 337

1.1. Semisimple groups 337

1.2. Factorization problem $\quad 338$

1.3. Total positivity 339

1.4. Generalized minors 339

1.5. The twist maps 340

1.6. Formulas for factorization parameters 341

1.7. Total positivity criteria 343

1.8. Fundamental determinantal identities 343

2. Preliminaries 344

2.1. Involutions 344

2.2. Commutation relations $\quad 345$

2.3. Generalized determinantal identities 346

2.4. Affine coordinates in Schubert cells 349

2.5. $y$-coordinates in double Bruhat cells 351

2.6. Factorization problem in Schubert cells 353

2.7. Totally positive bases for $N_{-}(w) \quad 354$

2.8. Total positivity in $y$-coordinates 356

3. Proofs of the main results 357

3.1. Proofs of Theorems 1.1, 1.2, and 1.3 357

3.2. Proofs of Theorems 1.6 and $1.7 \quad 359$

3.3. Proof of Theorem 1.9 360

3.4. Proofs of Theorems 1.11 and $1.12 \quad 363$

4. $G L_{n}$ theory 364

4.1. Bruhat cells and double Bruhat cells for $G L_{n} \quad 364$

4.2. Factorization problem for $G L_{n} \quad 365$

4.3. The twist maps for $G L_{n} \quad 367$

4.4. Double pseudoline arrangements $\quad 369$

4.5. Solution to the factorization problem 371

4.6. Applications to total positivity 374

References $\quad 379$

Received by the editors February 12, 1998.

1991 Mathematics Subject Classification. Primary 22E46; Secondary 05E15, 15A23.

Key words and phrases. Total positivity, semisimple groups, Bruhat decomposition.

The authors were supported in part by NSF grants \#DMS-9400914, \#DMS-9625511, and \#DMS-9700927, and by MSRI (NSF grant \#DMS-9022140). 


\section{INTRODUCTION}

This paper continues the algebraic study of total positivity in semisimple algebraic groups undertaken in $[3,4]$. Traditional theory of total positivity, pioneered in the 1930s by Gantmacher, Krein, and Schoenberg, studies matrices whose all minors are nonnegative. Recently, G. Lusztig [18] extended this classical subject by introducing the totally nonnegative variety $G_{\geq 0}$ in an arbitrary reductive group $G$. (Lusztig's study was motivated by surprising connections he discovered between total positivity and his theory of canonical bases for quantum groups.) The main object of study in $[3,4]$ was the structure of the intersection $G_{\geq 0} \cap N$, where $N$ is a maximal unipotent subgroup in $G$. In this paper we extend the results of $[3,4]$ to the whole variety $G_{\geq 0}$.

It turns out that the natural geometric framework for the study of $G_{\geq 0}$ is provided by the decomposition of $G$ into the disjoint union of double Bruhat cells $G^{u, v}=B u B \cap B_{-} v B_{-}$; here $B$ and $B_{-}$are two opposite Borel subgroups in $G$, and $u$ and $v$ belong to the Weyl group $W$ of $G$. We believe these double cells to be a very interesting object of study in their own right. The term "cells" might be misleading: in fact, the topology of $G^{u, v}$ is in general quite nontrivial. (In some special cases, the "real part" of $G^{u, v}$ was studied in [21, 22]. V. Deodhar [9] studied the intersections $B u B \cap B_{-} v B$ whose properties are very different from those of $G^{u, v}$.)

We study a family of birational parametrizations of $G^{u, v}$, one for each reduced expression $\mathbf{i}$ of the element $(u, v)$ in the Coxeter group $W \times W$. Every such parametrization can be thought of as a system of local coordinates in $G^{u, v}$. We call these coordinates the factorization parameters associated to i. They are obtained by expressing a generic element $x \in G^{u, v}$ as an element of the maximal torus $H=B \cap B_{-}$multiplied by the product of elements of various one-parameter subgroups in $G$ associated with simple roots and their negatives; the reduced expression $\mathbf{i}$ prescribes the order of factors in this product. The main technical result of this paper (Theorem 1.9) is an explicit formula for these factorization parameters as rational functions on the double Bruhat cell $G^{u, v}$.

Theorem 1.9 is formulated in terms of a special family of regular functions $\Delta_{\gamma, \delta}$ on the group $G$. These functions are suitably normalized matrix coefficients corresponding to pairs of extremal weights $(\gamma, \delta)$ in some fundamental representation of $G$. We believe these functions are very interesting subjects that merit further study. For the type $A$, they specialize to the minors of a matrix, and their properties are of course developed in great detail. It would be very interesting to extend the main body of the classical theory of determinantal identities to the family of functions $\Delta_{\gamma, \delta}$. In this paper, we make the first steps in this direction (see especially Theorems 1.16 and 1.17 below).

As in $[3,4]$, the main algebraic relations involving factorization parameters and generalized minors $\Delta_{\gamma, \delta}$ can be written in a "subtraction-free" form, and thus the theory can be developed over an arbitrary semifield. The readers familiar with $[3,4]$ will have no trouble extending the corresponding results there (cf. [3, Section 2]) to the more general context of this paper. We do not pursue this path here since at the moment we have not developed applications of this more general setup.

Returning to total positivity, our explicit formulas for factorization parameters allow us to obtain a family of total positivity criteria, each of which efficiently tests whether a given element $x$ from an arbitrary double Bruhat cell $G^{u, v}$ is totally 
nonnegative. More specifically, each of our criteria consists in verifying whether $x$ satisfies a system of inequalities of the form $\Delta_{\gamma, \delta}(x)>0$, the number of these inequalities being equal to the dimension of $G^{u, v}$ (see Theorem 1.11).

In Section 1 we give precise formulations of our main results. Their proofs are given in Sections 2 and 3.

The last Section 4 contains applications of our theory to the case of the general linear group. The case $G=G L_{n}$ is treated separately for a number of reasons. First, $G L_{n}$ is not a semisimple group (although everything reduces easily to $S L_{n}$ ). Furthermore, the questions that we consider become some very natural linear-algebraic questions whose understanding does not require any Lie-theoretic background. For instance, the factorization parameters become the parameters in factorizations of a square matrix into the smallest possible number of elementary Jacobi matrices. Our main results seem to be new even in this case. In Section 4, we tried to present them in an elementary form, making this section as self-contained as possible. Last but not least, our results in the $G L_{n}$ case have a particularly transparent formulation in the language of pseudoline arrangements.

The criteria of Theorem 1.11 lead us to new solutions of the classical problem of efficiently testing whether a given $n \times n$ matrix is totally positive, i.e., has all minors $>0$. This problem has a long history. The ground was broken in 1912 by M. Fekete [10] who proved that positivity of all solid minors, i.e., those formed by several consecutive rows and several consecutive columns, is sufficient for total positivity. It took a while before it was realized that Fekete's criterion was far from optimal: as shown in [13], it is enough to check the positivity of those solid minors that involve the first row or the first column of the matrix (this result can also be derived from [8]). Note that the number of such minors is $n^{2}$ while the total number of minors is $\left(\begin{array}{c}2 n \\ n\end{array}\right)-1$. One can show that at least $n^{2}$ minors are needed to characterize total positivity; thus the criterion reproduced above is "minimal". Theorem 4.13 (a specialization of Theorem 1.11) includes this criterion into a family of minimal total positivity criteria associated to shuffles of two reduced words for the permutation $w_{\mathrm{o}}=n n-1 \cdots 21$. For example, for $n=3$ we obtain 34 different criteria shown in Figure 8 at the end of the paper.

\section{MAin Results}

1.1. Semisimple groups. We begin by introducing general terminology and notation (mostly standard) for semisimple Lie groups and algebras (cf., e.g., [23]). Let $\mathfrak{g}$ be a semisimple complex Lie algebra of rank $r$ with the Cartan decomposition $\mathfrak{g}=\mathfrak{n}_{-} \oplus \mathfrak{h} \oplus \mathfrak{n}$. Let $e_{i}, h_{i}, f_{i}$, for $i=1, \ldots, r$, be the standard generators of $\mathfrak{g}$, and let $A=\left(a_{i j}\right)$ be the Cartan matrix. Thus $a_{i j}=\alpha_{j}\left(h_{i}\right)$, where $\alpha_{1}, \ldots, \alpha_{r} \in \mathfrak{h}^{*}$ are the simple roots of $\mathfrak{g}$. Let $G$ be a simply connected complex Lie group with the Lie algebra $\mathfrak{g}$. Let $N_{-}, H$ and $N$ be closed subgroups of $G$ with Lie algebras $\mathfrak{n}_{-}, \mathfrak{h}$ and $\mathfrak{n}$, respectively. Thus $H$ is a maximal torus, and $N$ and $N_{-}$are two opposite maximal unipotent subgroups of $G$. Let $B_{-}=H N_{-}$and $B=H N$ be the corresponding pair of opposite Borel subgroups. For $i=1, \ldots, r$ and $t \in \mathbb{C}$, we write

$$
x_{i}(t)=\exp \left(t e_{i}\right), \quad x_{\bar{i}}(t)=\exp \left(t f_{i}\right),
$$

so that $t \mapsto x_{i}(t)$ (resp. $\left.t \mapsto x_{\bar{i}}(t)\right)$ is a one-parameter subgroup in $N$ (resp. in $N_{-}$). We prefer the notation $x_{\bar{i}}(t)$ to the usual $y_{i}(t)$, for reasons that will become clear later. It will be convenient to denote $[1, r]=\{1, \ldots, r\}$ and $[\overline{1}, \bar{r}]=\{\overline{1}, \ldots, \bar{r}\}$. 
The weight lattice $P$ is the set of all weights $\gamma \in \mathfrak{h}^{*}$ such that $\gamma\left(h_{i}\right) \in \mathbb{Z}$ for all $i$. The group $P$ has a $\mathbb{Z}$-basis formed by the fundamental weights $\omega_{1}, \ldots, \omega_{r}$ defined by $\omega_{i}\left(h_{j}\right)=\delta_{i j}$. Every weight $\gamma \in P$ gives rise to a multiplicative character $a \mapsto a^{\gamma}$ of the maximal torus $H$; this character is given by $\exp (h)^{\gamma}=e^{\gamma(h)}(h \in \mathfrak{h})$.

The Weyl group $W$ of $G$ is defined by $W=\operatorname{Norm}_{G}(H) / H$. The action of $W$ on $H$ by conjugation gives rise to the action of $W$ on the weight lattice $P$ given by

$$
a^{w(\gamma)}=\left(w^{-1} a w\right)^{\gamma} \quad(w \in W, a \in H, \gamma \in P) .
$$

As usual, we identify $W$ with the corresponding group of linear transformations of $\mathfrak{h}^{*}$. The group $W$ is a Coxeter group generated by simple reflections $s_{1}, \ldots, s_{r}$ given by $s_{i}(\gamma)=\gamma-\gamma\left(h_{i}\right) \alpha_{i}$, for $\gamma \in \mathfrak{h}^{*}$.

A reduced word for $w \in W$ is a sequence of indices $\mathbf{i}=\left(i_{1}, \ldots, i_{m}\right)$ of shortest possible length $m$ such that $w=s_{i_{1}} \cdots s_{i_{m}}$. The number $m$ is denoted by $\ell(w)$ and is called the length of $w$. The set of reduced words for $w$ will be denoted by $R(w)$. The Weyl group $W$ has the unique element $w_{\mathrm{o}}$ of maximal length, and $\ell\left(w_{\mathrm{o}}\right)=\ell(w)+\ell\left(w^{-1} w_{\mathrm{o}}\right)$ for any $w \in W$.

1.2. Factorization problem. Recall that the group $G$ has two Bruhat decompositions, with respect to opposite Borel subgroups $B$ and $B_{-}$:

$$
G=\bigcup_{u \in W} B u B=\bigcup_{v \in W} B_{-} v B_{-} .
$$

The double Bruhat cells $G^{u, v}$ are defined by

$$
G^{u, v}=B u B \cap B_{-} v B_{-} ;
$$

thus $G$ is the disjoint union of all $G^{u, v}$ for $(u, v) \in W \times W$.

Theorem 1.1. The variety $G^{u, v}$ is biregularly isomorphic to a Zariski open subset of an affine space of dimension $r+\ell(u)+\ell(v)$.

We will study a family of birational parametrizations of $G^{u, v}$. To describe these parametrizations, we will need the following combinatorial notion.

A double reduced word for the elements $u, v \in W$ is a reduced word for an element $(u, v)$ of the Coxeter group $W \times W$. To avoid confusion, we will use the indices $\overline{1}, \overline{2}, \ldots, \bar{r}$ for the simple reflections in the first copy of $W$, and $1,2, \ldots, r$ for the second copy. A double reduced word for $(u, v)$ is nothing but a shuffle of a reduced word for $u$ written in the alphabet $[\overline{1}, \bar{r}]$ and a reduced word for $v$ written in the alphabet $[1, r]$. We denote the set of double reduced words for $(u, v)$ by $R(u, v)$.

For any sequence $\mathbf{i}=\left(i_{1}, \ldots, i_{m}\right)$ of indices from the alphabet $[1, r] \cup[\overline{1}, \bar{r}]$, consider the map $x_{\mathbf{i}}: H \times \mathbb{C}^{m} \rightarrow G$ defined by

$$
x_{\mathbf{i}}\left(a ; t_{1}, \ldots, t_{m}\right)=a x_{i_{1}}\left(t_{1}\right) \cdots x_{i_{m}}\left(t_{m}\right),
$$

where we use the notation of (1.1).

Let $\mathbb{C}_{\neq 0}$ denote the set of nonzero complex numbers.

Theorem 1.2. For any $u, v \in W$ and $\mathbf{i}=\left(i_{1}, \ldots, i_{m}\right) \in R(u, v)$, the map $x_{\mathbf{i}}$ restricts to a biregular isomorphism between $H \times \mathbb{C}_{\neq 0}^{m}$ and a Zariski open subset of the double Bruhat cell $G^{u, v}$.

Thus $x_{\mathbf{i}}$ gives rise to a birational isomorphism between $H \times \mathbb{C}^{m}$ and $G^{u, v}$. We remark that this property holds if and only if $\mathbf{i}$ is a double reduced word for $(u, v)$. 
Theorem 1.2 tells us that for a generic element $x \in G^{u, v}$ and any $\mathbf{i}=\left(i_{1}, \ldots, i_{m}\right)$ $\in R(u, v)$, there are uniquely defined factorization parameters $a, t_{1}, \ldots, t_{m}$ such that

$$
x=a x_{i_{1}}\left(t_{1}\right) \cdots x_{i_{m}}\left(t_{m}\right) .
$$

One of our main results is the solution of the following factorization problem: find explicit formulas for the inverse birational isomorphism $x_{\mathbf{i}}^{-1}$ between $G^{u, v}$ and $H \times \mathbb{C}^{m}$. In other words, we express the factorization parameters in terms of the element $x$ and the double reduced word $\mathbf{i}$ underlying the factorization. Our solution of the factorization problem generalizes Theorems 1.4 and 6.2 in [4] (the case of $x \in N$ ), which in turn generalize Theorems 1.4 and 5.4.2 in [3] (same, for type $A$ ).

1.3. Total positivity. We will apply our solution of the factorization problem to the study of total positivity. Following G. Lusztig [18], let us define totally nonnegative elements in $G$. Let $H_{>0}$ be the subgroup of $H$ consisting of all $a \in$ $H$ such that $a^{\gamma} \in \mathbb{R}_{>0}$ for any weight $\gamma \in P$. (We denote by $\mathbb{R}_{>0}$ the set of positive reals.) The set $G_{\geq 0}$ of totally nonnegative elements is, by definition, the multiplicative semigroup in $G$ generated by $H_{>0}$ and the elements $x_{i}(t)$ and $x_{\bar{i}}(t)$, for $i \in[1, r]$ and $t \in \mathbb{R}_{>0}$. It is easy to see that a totally nonnegative element $x \in G$ can be represented as $x=x_{\mathbf{i}}\left(a ; t_{1}, \ldots, t_{m}\right)$, for some sequence $\mathbf{i}$, with all the $t_{k}$ positive and $a \in H_{>0}$. For the type $A_{r}$ (i.e., for $G=S L_{n}(\mathbb{C}), n=r+1$ ), a theorem in [17], based on a result by A. Whitney [24], tells us that the above definition of total nonnegativity coincides with the usual one $[2,16]$ : a matrix (with determinant 1 ) is totally nonnegative if and only if all its minors are nonnegative.

The set $G_{\geq 0}$ is the disjoint union of the subsets $G_{>0}^{u, v}$ obtained by intersecting it with double Bruhat cells:

$$
G_{>0}^{u, v}=G_{\geq 0} \cap G^{u, v} .
$$

We call the $G_{>0}^{u, v}$ totally positive varieties; they will be one of the main objects of study in this paper. The terminology is justified by the following observation made by Lusztig [18]: in the special case $G=S L_{n}(\mathbb{C})$ and $u=v=w_{\mathrm{o}}$, the variety $G_{>0}^{u, v}$ is the set of all $n \times n$-matrices (with determinant 1 ) which are (strictly) totally positive in the usual sense, i.e., all their minors are positive. We note that the decomposition $G_{\geq 0}=\bigcup G_{>0}^{u, v}$ appeared in [18] (without explicit mentioning of double Bruhat cells). The following theorem can be derived from the results in [18].

Theorem 1.3. For any $u, v \in W$ and any double reduced word $\mathbf{i} \in R(u, v)$, the map $x_{\mathbf{i}}$ restricts to a bijection $H_{>0} \times \mathbb{R}_{>0}^{m} \rightarrow G_{>0}^{u, v}$.

Informally speaking, Theorem 1.3 asserts that an element $x \in G^{u, v}$ is totally nonnegative if and only if for some (equivalently, any) double reduced word $\mathbf{i} \in$ $R(u, v)$, the factorization parameters $a, t_{1}, \ldots, t_{m}$ appearing in (1.4) are well defined and positive. Thus the solution of the factorization problem will lead to a family of total positivity criteria - one for each double reduced word.

1.4. Generalized minors. The main ingredients of our answer to the factorization problem are similar to those in [4]: a family of regular functions on $G$ generalizing minors of a square matrix, and a biregular "twist" $G^{u, v} \rightarrow G^{u^{-1}, v^{-1}}$. 
We will denote by $G_{0}=N_{-} H N$ the set of elements $x \in G$ that have Gaussian decomposition; this decomposition will be written as

$$
x=[x]_{-}[x]_{0}[x]_{+} .
$$

Following [4], for any fundamental weight $\omega_{i}$, let $\Delta^{\omega_{i}}$ denote the regular function on $G$ whose restriction to the open set $G_{0}$ is given by

$$
\Delta^{\omega_{i}}(x)=[x]_{0}^{\omega_{i}} .
$$

For the type $A_{r}$, the $\Delta^{\omega_{i}}(x)$ are the principal minors of a matrix $x$. We will use the same terminology in the general case as well.

To define the analogues of arbitrary minors, we will need two special representatives $\bar{w}, \overline{\bar{w}} \in G$ for any element $w \in W$. For a simple reflection $s_{i}$, set

$$
\overline{s_{i}}=\varphi_{i}\left(\begin{array}{cc}
0 & -1 \\
1 & 0
\end{array}\right), \quad \overline{\overline{s_{i}}}=\varphi_{i}\left(\begin{array}{cc}
0 & 1 \\
-1 & 0
\end{array}\right),
$$

where $\varphi_{i}: S L_{2} \rightarrow G$ is the group homomorphism given by

$$
\varphi_{i}\left(\begin{array}{ll}
1 & t \\
0 & 1
\end{array}\right)=x_{i}(t), \quad \varphi_{i}\left(\begin{array}{ll}
1 & 0 \\
t & 1
\end{array}\right)=x_{\bar{i}}(t) .
$$

Alternatively, we could define

$$
\overline{s_{i}}=x_{i}(-1) x_{\bar{i}}(1) x_{i}(-1), \quad \overline{\overline{s_{i}}}=x_{i}(1) x_{\bar{i}}(-1) x_{i}(1) .
$$

It is known (and easy to check) that the families $\left\{\overline{s_{i}}\right\}$ and $\left\{\overline{\overline{s_{i}}}\right\}$ satisfy the braid relations in $W$. It follows that the representatives $\bar{w}$ and $\overline{\bar{w}}$ can be uniquely and unambiguously defined for any $w \in W$ by the condition that

$$
\overline{w^{\prime} w^{\prime \prime}}=\overline{w^{\prime}} \cdot \overline{w^{\prime \prime}}, \overline{\overline{w^{\prime} w^{\prime \prime}}}=\overline{\overline{w^{\prime}}} \cdot \overline{\overline{w^{\prime \prime}}}
$$

whenever $\ell\left(w^{\prime} w^{\prime \prime}\right)=\ell\left(w^{\prime}\right)+\ell\left(w^{\prime \prime}\right)$.

Definition 1.4. For $u, v \in W$, define a regular function $\Delta_{u \omega_{i}, v \omega_{i}}$ on $G$ by setting

$$
\Delta_{u \omega_{i}, v \omega_{i}}(x)=\Delta^{\omega_{i}}\left(\overline{\overline{u^{-1}}} x \bar{v}\right)
$$

One has to check that this is well defined, i.e., the right-hand side of (1.10) only depends on the weights $u \omega_{i}$ and $v \omega_{i}$, not on the particular choice of $u$ and $v$. This is done in Section 2.3 (cf. Proposition 2.3).

For the type $A_{r}$, the functions $\Delta_{u \omega_{i}, v \omega_{i}}(x)$ are the minors of a matrix $x$. In the general case, we will refer to them as generalized minors, or simply as minors if there will be no danger of confusion.

1.5. The twist maps. To define the twist maps, we will need the involutive automorphism $x \mapsto x^{\theta}$ of the group $G$ which is uniquely determined by

$$
a^{\theta}=a^{-1} \quad(a \in H), \quad x_{i}(t)^{\theta}=x_{\bar{i}}(t), \quad x_{\bar{i}}(t)^{\theta}=x_{i}(t) .
$$

Notice that the involution $\theta$ preserves total nonnegativity. For the type $A_{r}$, if $x$ is a matrix with determinant 1 , then the matrix $x^{\theta}$ is formed by signless cofactors of $x$; in other words, the $(i, j)$-entry of $x^{\theta}$ is simply the minor of $x$ obtained by deleting the $i$ th row and the $j$ th column.

Definition 1.5. For any $u, v \in W$, the twist map $\zeta^{u, v}: x \mapsto x^{\prime}$ is defined by

$$
x^{\prime}=\left(\left[\overline{\overline{u^{-1}}} x\right]_{-}^{-1} \overline{\overline{u^{-1}}} x \overline{v^{-1}}\left[x \overline{v^{-1}}\right]_{+}^{-1}\right)^{\theta} .
$$


Theorem 1.6. The right-hand side of (1.12) is well defined for any $x \in G^{u, v}$, and the twist map $\zeta^{u, v}$ establishes a biregular isomorphism between $G^{u, v}$ and $G^{u^{-1}, v^{-1}}$. The inverse isomorphism is $\zeta^{u^{-1}, v^{-1}}$.

The specific choice of representatives for $u$ and $v$ in (1.12) is essential for the following important property.

Theorem 1.7. The twist map $\zeta^{u, v}$ restricts to a bijection $G_{>0}^{u, v} \rightarrow G_{>0}^{u^{-1}, v^{-1}}$.

Example 1.8. Let $G=S L_{2}(\mathbb{C})$. Then $W=S_{2}$ consists of two permutations: $e$ and $w_{\mathrm{o}}$; thus $G$ is partitioned into four double Bruhat cells. Table 1 shows the conditions under which a matrix $x=\left(\begin{array}{ll}x_{11} & x_{12} \\ x_{21} & x_{22}\end{array}\right)$ with determinant 1 belongs to each of these cells, or to the corresponding totally positive variety $G_{>0}^{u, v}$. The table also shows the formulas defining each twist map $\zeta^{u, v}$.

TABLE 1. Double Bruhat decomposition and the twist maps for $S L_{2}$

\begin{tabular}{c|cccc} 
& $u=e$ & $u=e$ & $u=w_{\mathrm{o}}$ & $u=w_{\mathrm{o}}$ \\
& $v=e$ & $v=w_{\mathrm{o}}$ & $v=e$ & $v=w_{\mathrm{o}}$ \\
\hline \multirow{2}{*}{$G^{u, v}$} & $x_{12}=0$ & $x_{12} \neq 0$ & $x_{12}=0$ & $x_{12} \neq 0$ \\
& $x_{21}=0$ & $x_{21}=0$ & $x_{21} \neq 0$ & $x_{21} \neq 0$ \\
\hline \multirow{3}{*}{$G_{>0}^{u, v}$} & $x_{11}>0$ & $x_{11}>0$ & $x_{11}>0$ & $x_{11}>0$ \\
& $x_{12}=0$ & $x_{12}>0$ & $x_{12}=0$ & $x_{12}>0$ \\
& $x_{21}=0$ & $x_{21}=0$ & $x_{21}>0$ & $x_{21}>0$ \\
\hline$\zeta^{u, v}(x)$ & $\left(\begin{array}{cc}x_{11}^{-1} & 0 \\
0 & x_{11}\end{array}\right)$ & $\left(\begin{array}{cc}x_{12}^{-1} & x_{11}^{-1} \\
0 & x_{12}\end{array}\right)$ & $\left(\begin{array}{cc}x_{21}^{-1} & 0 \\
x_{11}^{-1} & x_{21}\end{array}\right)$ & $\left(\begin{array}{ccc}x_{11} x_{12}^{-1} x_{21}^{-1} & x_{21}^{-1} \\
x_{12}^{-1} & x_{22}\end{array}\right)$
\end{tabular}

1.6. Formulas for factorization parameters. To give explicit formulas for factorization parameters, we will need some more notation. First, we will write

$$
|i|=|\bar{i}|=i, \varepsilon(i)=1, \varepsilon(\bar{i})=-1,
$$

for any $i \in[1, r]$. Let us fix a pair $(u, v) \in W \times W$ and a double reduced word $\mathbf{i}=\left(i_{1}, \ldots, i_{m}\right) \in R(u, v)$. Recall that $\mathbf{i}$ is a shuffle of a reduced word for $u$ written in the alphabet $[\overline{1}, \bar{r}]$ and a reduced word for $v$ written in the alphabet $[1, r]$. In particular, the length $m$ of $\mathbf{i}$ is equal to $\ell(u)+\ell(v)$. We will add $r$ additional entries $i_{m+1}, \ldots, i_{m+r}$ at the end of $\mathbf{i}$ by setting

$$
i_{m+j}=\bar{j} \quad(j \in[1, r]) .
$$

For $k \in[1, m+r]$, let us denote

$$
u_{\geq k}=\prod_{\substack{l=m, \ldots, k \\ i_{l} \in[\overline{1}, \bar{r}]}} s_{\left|i_{l}\right|}, \quad v_{<k}=\prod_{\substack{l=1, \ldots, k-1 \\ i_{l} \in[1, r]}} s_{\left|i_{l}\right|} .
$$

This notation means that in the first (resp. second) product in (1.15), the index $l$ is decreasing (resp. increasing); by convention, we also have $u_{\geq k}=e$ and $v_{<k}=v$ for $k>m$. (For example, if $\mathbf{i}=\overline{2} 1 \overline{3} 32 \overline{1} \overline{2} 1 \overline{1}$, then, say, $u_{\geq 7}=s_{1} s_{2}$ and $v_{<7}=s_{1} s_{3} s_{2}$.) Let us define a regular function $\Delta_{k}=\Delta_{k, \mathbf{i}}$ on $G$ by

$$
\Delta_{k}=\Delta_{k, \mathbf{i}}=\Delta_{u_{\geq k} \omega_{\left|i_{k}\right|}, v_{<k} \omega_{\left|i_{k}\right|}} .
$$


For $k \in[1, m+r]$, we denote

$$
k^{-}=\max \left\{l: l<k,\left|i_{l}\right|=\left|i_{k}\right|\right\}
$$

unless $\left|i_{l}\right| \neq\left|i_{k}\right|$ for all $l<k$, in which case we set $k^{-}=0$. For $k \in[1, m+r]$ and $l \in[0, m+r]$, let

$$
\chi(k, l)= \begin{cases}1 & \text { if } k<l \text { and } \varepsilon\left(i_{k}\right)=\varepsilon\left(i_{l}\right) \\ 1 / 2 & \text { if } k=l \\ 0 & \text { if } k>l \text { or } \varepsilon\left(i_{k}\right) \neq \varepsilon\left(i_{l}\right)\end{cases}
$$

With all this notation in mind, we now formulate our first main result: a solution to the factorization problem of Section 1.2.

Theorem 1.9. Let $\mathbf{i}=\left(i_{1}, \ldots, i_{m}\right)$ be a double reduced word for $(u, v)$, and suppose an element $x \in G^{u, v}$ can be factored as $x=a x_{i_{1}}\left(t_{1}\right) \cdots x_{i_{m}}\left(t_{m}\right)$, with $a \in H$ and all $t_{k}$ nonzero complex numbers. Then the factorization parameters are determined by the following formulas:

$$
\begin{aligned}
& t_{k}=\prod_{l=1}^{m+r} \Delta_{l, \mathbf{i}}\left(x^{\prime}\right)^{\left(\chi\left(k, l^{-}\right)-\chi(k, l)\right) a_{\left|i_{l}\right|,\left|i_{k}\right|}} ; \\
& a^{\omega_{i}}=\prod_{\substack{1 \leq k \leq m+r \\
\left|i_{k}\right|=i, \varepsilon\left(i_{k}\right) \neq \varepsilon\left(i_{k^{-}}\right)}} \Delta_{k, \mathbf{i}\left(x^{\prime}\right)^{\varepsilon\left(i_{k}\right)},}
\end{aligned}
$$

where $x^{\prime}=\zeta^{u, v}(x)$, and we use the convention $\varepsilon\left(i_{0}\right)=1$.

Formulas (1.19) can be restated as the following closed expression for the element $a$ :

$$
a=\prod_{\substack{1 \leq k \leq m+1 \\ \varepsilon\left(i_{k}\right) \neq \varepsilon\left(i_{k-1}\right)}}\left[\overline{\overline{u_{\geq k}^{-1}}} x^{\prime} \overline{v_{<k}}\right]_{0}^{\varepsilon\left(i_{k}\right)} .
$$

Since (1.18) and (1.19) express the $m+r$ independent parameters $t_{1}, \ldots, t_{m}$ and $a^{\omega_{1}}, \ldots, a^{\omega_{r}}$ as Laurent monomials in the $m+r$ minors $\Delta_{1, \mathbf{i}}\left(x^{\prime}\right), \ldots, \Delta_{m+r, \mathbf{i}}\left(x^{\prime}\right)$, we obtain the following important corollary.

Theorem 1.10. Under the assumptions of Theorem 1.9, the parameters $t_{1}, \ldots, t_{m}$ and $a^{\omega_{1}}, \ldots, a^{\omega_{r}}$ are related to the minors $\Delta_{1, \mathbf{i}}\left(x^{\prime}\right), \ldots, \Delta_{m+r, \mathbf{i}}\left(x^{\prime}\right)$ by an invertible monomial transformation.

The inverse of this monomial transformation can be computed explicitly: one can show that it is given by

$$
\Delta_{k, \mathbf{i}}\left(x^{\prime}\right)=a^{-u u_{\geq k} \omega_{\left|i_{k}\right|}} \prod_{\substack{l<k \\ i_{l} \in[1, r]}} t_{l}^{u_{\geq l}^{-1} u_{\geq k} \omega_{\left|i_{k}\right|}\left(h_{\left|i_{l}\right|}\right)} \prod_{\substack{l \geq k \\ i_{l} \in[1, r]}} t_{l}^{v_{l}^{-1} l_{+1} v_{<k} \omega_{\left|i_{k}\right|}\left(h_{\left|i_{l}\right|}\right)} .
$$

Since we will not use this formula, we will not prove it in this paper. 
1.7. Total positivity criteria. Theorem 1.9 implies a family of criteria for total positivity that generalize the ones in $[3,4]$. Each of these criteria asserts that a point $x \in G^{u, v}$ belongs to the totally positive variety $G_{>0}^{u, v}$ if and only if a particular collection of $\ell(u)+\ell(v)+r$ minors evaluated at $x$ are all positive.

For every double reduced word $\mathbf{i}=\left(i_{1}, \ldots, i_{m}\right) \in R(u, v)$, let $F(\mathbf{i})$ denote the following collection of $m+r$ minors:

$$
F(\mathbf{i})=\left\{\Delta_{k, \mathbf{i}^{*}}: k \in[1, m+r]\right\}
$$

(cf. (1.16)), where $\mathbf{i}^{*}=\left(i_{m}, \ldots, i_{1}\right) \in R\left(u^{-1}, v^{-1}\right)$ is $\mathbf{i}$ written backwards.

Theorem 1.11. Let $\mathbf{i} \in R(u, v)$. An element $x \in G^{u, v}$ is totally nonnegative if and only if $\Delta(x)>0$ for any minor $\Delta \in F(\mathbf{i})$.

As a consequence, for any two double reduced words $\mathbf{i}, \mathbf{i}^{\prime} \in R(u, v)$, the positivity of all minors from $F(\mathbf{i})$ at a given $x \in G^{u, v}$ is equivalent to the positivity of all minors from $F\left(\mathbf{i}^{\prime}\right)$. This phenomenon has the following algebraic explanation. Let

$$
F(u, v)=\bigcup_{\mathbf{i} \in R(u, v)} F(\mathbf{i}) .
$$

This can be restated as

$$
F(u, v)=\left\{\Delta_{u^{\prime} \omega_{i}, v^{\prime} \omega_{i}}: i \in[1, r], u^{\prime} \preceq u, v^{\prime} \preceq v^{-1}\right\},
$$

where $u^{\prime} \preceq u$ stands for $\ell(u)=\ell\left(u^{\prime}\right)+\ell\left(u^{\prime-1} u\right.$ ) (the weak order on $W$ ).

Theorem 1.12. For any $\mathbf{i} \in R(u, v)$, the collection $F(\mathbf{i})$ is a transcendence basis for the field of rational functions $\mathbb{C}\left(G^{u, v}\right)$. Furthermore, every minor in $F(u, v)$ can be expressed as a ratio of two polynomials in the variables $\Delta \in F(\mathbf{i})$ with nonnegative integer coefficients.

Combining Theorems 1.2, 1.6, and 1.10 yields the following result.

Theorem 1.13. For any $\mathbf{i} \in R(u, v)$, every minor in $F(u, v)$ is a Laurent polynomial with integer coefficients in the variables $\Delta \in F(\mathbf{i})$.

We suggest the following common refinement of Theorems 1.12 and 1.13.

Conjecture 1.14. For any $\mathbf{i} \in R(u, v)$, every minor in $F(u, v)$ is a Laurent polynomial in the variables $\Delta \in F(\mathbf{i})$ with nonnegative integer coefficients.

Note that Theorems 1.12 and 1.13 do not automatically imply Conjecture 1.14, since there do exist subtraction-free rational expressions that are Laurent polynomials although not with nonnegative coefficients (for example, think of $\left(p^{3}+q^{3}\right)$ / $\left.(p+q)=p^{2}-p q+q^{2}\right)$.

It is not hard to derive the following special case of Conjecture 1.14 from [3, Theorem 3.7.4].

Theorem 1.15. Conjecture 1.14 holds for $G=S L_{r+1}$, when either $u$ or $v$ is the identity element $e$.

1.8. Fundamental determinantal identities. The subtraction-free rational expressions in Theorem 1.12 can be computed by an explicitly described algorithm. This algorithm is based on repeated application of the following generalized determinantal identities.

The first group of identities follows from [4, Corollary 6.6]. They correspond to pairs of simple roots that generate a root subsystem of type $A_{2}$ or $B_{2}$. There are 
similar identities for subsystems of type $G_{2}$ (see [4, (4.8)-(4.11)]) although we will not reproduce them here.

Theorem 1.16. Let $u, v \in W$ and $i, j \in[1, r]$.

(1) If $a_{i j}=a_{j i}=-1$ and $\ell\left(v s_{i} s_{j} s_{i}\right)=\ell(v)+3$, then

$$
\Delta_{u \omega_{i}, v s_{i} \omega_{i}} \Delta_{u \omega_{j}, v s_{j} \omega_{j}}=\Delta_{u \omega_{i}, v \omega_{i}} \Delta_{u \omega_{j}, v s_{i} s_{j} \omega_{j}}+\Delta_{u \omega_{i}, v s_{j} s_{i} \omega_{i}} \Delta_{u \omega_{j}, v \omega_{j}} .
$$

(2) If $a_{i j}=-2, a_{j i}=-1$, and $\ell\left(v s_{i} s_{j} s_{i} s_{j}\right)=\ell(v)+4$, then

$$
\begin{aligned}
& \Delta_{u \omega_{j}, v s_{i} s_{j} \omega_{j}} \Delta_{u \omega_{i}, v s_{j} s_{i} \omega_{i}}^{2} \Delta_{u \omega_{j}, v s_{j} \omega_{j}} \\
& =\Delta_{u \omega_{j}, v s_{j} s_{i} s_{j} \omega_{j}} \Delta_{u \omega_{i}, v s_{j} s_{i} \omega_{i}}^{2} \Delta_{u \omega_{j}, v \omega_{j}}^{2} \\
& +\left(\Delta_{u \omega_{i}, v \omega_{i}} \Delta_{u \omega_{j}, v s_{j} s_{i} s_{j} \omega_{j}}+\Delta_{u \omega_{i}, v s_{i} s_{j} s_{i} \omega_{i}} \Delta_{u \omega_{j}, v s_{j} \omega_{j}}\right)^{2}
\end{aligned}
$$

and

$$
\begin{aligned}
& \Delta_{u \omega_{i}, v s_{i} \omega_{i}} \Delta_{u \omega_{i}, v s_{j} s_{i} \omega_{i}} \Delta_{u \omega_{j}, v s_{j} \omega_{j}} \\
& =\Delta_{u \omega_{i}, v s_{j} s_{i} \omega_{i}}^{2} \Delta_{u \omega_{j}, v \omega_{j}} \\
& \quad+\Delta_{u \omega_{i}, v \omega_{i}}\left(\Delta_{u \omega_{i}, v \omega_{i}} \Delta_{u \omega_{j}, v s_{j} s_{i} s_{j} \omega_{j}}+\Delta_{u \omega_{i}, v s_{i} s_{j} s_{i} \omega_{i}} \Delta_{u \omega_{j}, v s_{j} \omega_{j}}\right) .
\end{aligned}
$$

(3) Each of the above identities has a companion identity, obtained by "transposing" all participating minors, i.e., by replacing every $\Delta_{\gamma, \delta}$ by $\Delta_{\delta, \gamma}$.

We also make use of the following new identity.

Theorem 1.17. Suppose $u, v \in W$ and $i \in[1, r]$ are such that $\ell\left(u s_{i}\right)=\ell(u)+1$ and $\ell\left(v s_{i}\right)=\ell(v)+1$. Then

$$
\Delta_{u \omega_{i}, v \omega_{i}} \Delta_{u s_{i} \omega_{i}, v s_{i} \omega_{i}}=\Delta_{u s_{i} \omega_{i}, v \omega_{i}} \Delta_{u \omega_{i}, v s_{i} \omega_{i}}+\prod_{j \neq i} \Delta_{u \omega_{j}, v \omega_{j}}^{-a_{j i}} .
$$

The proof of Theorem 1.17 is given in Section 2.3.

For the type $A_{r}$, the identities of Theorems 1.16 and 1.17 become certain 3term determinantal identities known since the early 19th century. We discuss their attribution in Section 4.6.

\section{Preliminaries}

In what follows, we retain the notation and terminology introduced in Section 1.

2.1. Involutions. Following [4], we define involutive anti-automorphisms $x \mapsto x^{T}$ (the "transpose") and $x \mapsto x^{\iota}$ of the group $G$ by setting

$$
a^{T}=a \quad(a \in H), \quad x_{i}(t)^{T}=x_{\bar{i}}(t), \quad x_{\bar{i}}(t)^{T}=x_{i}(t)
$$

and

$$
a^{\iota}=a^{-1} \quad(a \in H), \quad x_{i}(t)^{\iota}=x_{i}(t), \quad x_{\bar{i}}(t)^{\iota}=x_{\bar{i}}(t) .
$$

These two involutive anti-automorphisms commute with each other and with the involutive anti-automorphism $x \mapsto x^{-1}$ of $G$. Hence these three maps generate the group isomorphic to $(\mathbb{Z} / 2 \mathbb{Z})^{3}$; in particular, any composition of them is again an involution. Notice that the involutions $x \mapsto x^{T}$ and $x \mapsto x^{\iota}$ preserve total nonnegativity, while $x \mapsto x^{-1}$ does not. Informally, $x^{\iota}$ is a "totally nonnegative version" of $x^{-1}$.

In the notation just introduced, the involution $x \mapsto x^{\theta}$ that was defined by (1.11) is given by $x^{\theta}=\left(x^{\iota}\right)^{T}=\left(x^{T}\right)^{\iota}$. 
The involutions $x \mapsto\left(x^{-1}\right)^{\iota}$ and $x \mapsto x^{T}$ obviously preserve $G_{0}=N_{-} H N$, and we have

$$
\left[\left(x^{-1}\right)^{\iota}\right]_{0}=\left[x^{T}\right]_{0}=[x]_{0} .
$$

All three involutions $x \mapsto x^{-1}, x \mapsto x^{T}$ and $x \mapsto x^{\iota}$ act on $W$ by $w \mapsto w^{-1}$. The relations between these involutions and the special representatives introduced in Section 1.4 are summarized in the following proposition.

Proposition 2.1. We have

$$
\bar{w}^{-1}=\bar{w}^{T}=\overline{\overline{w^{-1}}}=\overline{\bar{w}}^{\iota}, \quad \overline{w^{-1}}=\bar{w}^{\iota}=\overline{\bar{w}}^{-1}=\overline{\bar{w}}^{T} .
$$

Proof. Since all three involutions are anti-automorphisms, it is enough to check (2.4) for $w=s_{i}$, in which case it follows by a calculation in $S L_{2}$.

2.2. Commutation relations. For convenience of exposition, we collect here some known commutation relations in $G$ that will be used in our proofs. Recall that $x_{i}(t)$ and $x_{\bar{i}}(t)$ are defined by (1.1), and $\alpha_{1}, \ldots, \alpha_{r}$ are the simple roots of $\mathfrak{g}$.

First of all, for every $a \in H$, we have

$$
a x_{i}(t)=x_{i}\left(a^{\alpha_{i}} t\right) a, \quad a x_{\bar{i}}(t)=x_{\bar{i}}\left(a^{-\alpha_{i}} t\right) a .
$$

The following relations between the elements $x_{i}(t)$ can be found, e.g., in [4, Section 3] (some of them appeared earlier in [19]). If $a_{i j}=a_{j i}=0$, then

$$
x_{i}\left(t_{1}\right) x_{j}\left(t_{2}\right)=x_{j}\left(t_{2}\right) x_{i}\left(t_{1}\right),
$$

for any $t_{1}$ and $t_{2}$. If $a_{i j}=a_{j i}=-1$, then

$$
x_{i}\left(t_{1}\right) x_{j}\left(t_{2}\right) x_{i}\left(t_{3}\right)=x_{j}\left(\frac{t_{2} t_{3}}{t_{1}+t_{3}}\right) x_{i}\left(t_{1}+t_{3}\right) x_{j}\left(\frac{t_{1} t_{2}}{t_{1}+t_{3}}\right)
$$

whenever $t_{1}+t_{3} \neq 0$. If $a_{i j}=-2$ and $a_{j i}=-1$, then

$$
x_{i}\left(t_{1}\right) x_{j}\left(t_{2}\right) x_{i}\left(t_{3}\right) x_{j}\left(t_{4}\right)=x_{j}\left(t_{2} t_{3}^{2} t_{4} q^{-1}\right) x_{i}\left(q p^{-1}\right) x_{j}\left(p^{2} q^{-1}\right) x_{i}\left(t_{1} t_{2} t_{3} p^{-1}\right),
$$

where

$$
p=t_{1} t_{2}+\left(t_{1}+t_{3}\right) t_{4}, q=t_{1}^{2} t_{2}+\left(t_{1}+t_{3}\right)^{2} t_{4} ;
$$

this relation holds whenever $p \neq 0$ and $q \neq 0$. In the case when $a_{i j}=-3, a_{j i}=$ -1 (i.e., when $\alpha_{i}$ and $\alpha_{j}$ generate a root subsystem of type $G_{2}$ ), there is also a relation similar to (2.7) and (2.8). This relation is given in $[4,(3.6)-(3.10)]$; we will not reproduce it here. Each of the relations (2.6)-(2.8) has a counterpart for the elements $x_{\bar{i}}(t)$; it can be obtained by applying the anti-automorphism $x \mapsto x^{T}$ (cf. (2.1)).

In conclusion, let us describe the commutation relations between the elements $x_{i}(t)$ and $x_{\bar{j}}\left(t^{\prime}\right)$. If $i \neq j$, then $\left[e_{i}, f_{j}\right]=0$ in $\mathfrak{g}$, hence

$$
x_{i}(t) x_{\bar{j}}\left(t^{\prime}\right)=x_{\bar{j}}\left(t^{\prime}\right) x_{i}(t),
$$

for any $t$ and $t^{\prime}$. To handle the case $i=j$, we will need the following notation. For a nonzero $t \in \mathbb{C}$ and $i \in[1, r]$, we denote

$$
t^{h_{i}}=\varphi_{i}\left(\begin{array}{cc}
t & 0 \\
0 & t^{-1}
\end{array}\right)
$$


where $\varphi_{i}: S L_{2} \rightarrow G$ is defined by (1.7); alternatively, $t^{h_{i}}$ is an element of $H$ uniquely determined by the condition that $\left(t^{h_{i}}\right)^{\gamma}=t^{\gamma\left(h_{i}\right)}$ for any weight $\gamma \in P$. Then we have

$$
x_{i}(t) x_{\bar{i}}\left(t^{\prime}\right)=x_{\bar{i}}\left(\frac{t^{\prime}}{1+t t^{\prime}}\right)\left(1+t t^{\prime}\right)^{h_{i}} x_{i}\left(\frac{t}{1+t t^{\prime}}\right)
$$

whenever $1+t t^{\prime} \neq 0$. This relation can be first checked for $S L_{2}$ by a simple matrix calculation, and then extended to $G$ by applying the homomorphism $\varphi_{i}$. By the same method, we verify the relations

$$
x_{i}(t) x_{\bar{i}}\left(-t^{-1}\right)=x_{\bar{i}}\left(t^{-1}\right) t^{h_{i}} \overline{\overline{s_{i}}}
$$

and

$$
\begin{aligned}
& x_{i}(t) \overline{s_{i}}=x_{\bar{i}}\left(t^{-1}\right) t^{h_{i}} x_{i}\left(-t^{-1}\right), \\
& \overline{\overline{s_{i}}} x_{\bar{i}}(t)=x_{\bar{i}}\left(-t^{-1}\right) t^{h_{i}} x_{i}\left(t^{-1}\right) .
\end{aligned}
$$

2.3. Generalized determinantal identities. We start with some identities for the "principal minors" $\Delta^{\omega_{i}}$. The definition (1.6) implies that, for any $x \in G$, $x^{-} \in N_{-}, x^{+} \in N$, and $a \in H$, we have

$$
\begin{aligned}
& \Delta^{\omega_{i}}\left(x^{-} x\right)=\Delta^{\omega_{i}}\left(x x^{+}\right)=\Delta^{\omega_{i}}(x), \\
& \Delta^{\omega_{i}}(a x)=\Delta^{\omega_{i}}(x a)=a^{\omega_{i}} \Delta^{\omega_{i}}(x) .
\end{aligned}
$$

In view of (2.3), we also have

$$
\Delta^{\omega_{i}}\left(\left(x^{-1}\right)^{\iota}\right)=\Delta^{\omega_{i}}\left(x^{T}\right)=\Delta^{\omega_{i}}(x) .
$$

The following property is less obvious.

Proposition 2.2. For any $x \in G, j \neq i$, and $t \in \mathbb{C}$, we have

$$
\Delta^{\omega_{i}}\left(x x_{\bar{j}}(t)\right)=\Delta^{\omega_{i}}\left(x_{j}(t) x\right)=\Delta^{\omega_{i}}(x) .
$$

Proof. It is possible to deduce the proposition from the commutation relations given in Section 2.2 but we prefer another proof based on representation theory. The group $G$ acts by right translations in the space $\mathbb{C}[G]$ of regular functions on $G$. It is well known that every $f \in \mathbb{C}[G]$ generates a finite-dimensional subrepresentation of $\mathbb{C}[G]$. In view of $(2.14)$, the function $\Delta^{\omega_{i}}$ is a highest weight vector of weight $\omega_{i}$ in $\mathbb{C}[G]$. Since $\omega_{i}\left(h_{j}\right)=0$ for $j \neq i$, it follows that $\Delta^{\omega_{i}}$ has weight 0 with respect to the subgroup $\varphi_{j}\left(S L_{2}\right)$ of $G$ (cf. (1.7)). Therefore, $\Delta^{\omega_{i}}$ generates a trivial representation of $\varphi_{j}\left(S L_{2}\right)$. In particular, $\Delta^{\omega_{i}}\left(x x_{\bar{j}}(t)\right)=\Delta^{\omega_{i}}(x)$, as desired. The equality $\Delta^{\omega_{i}}\left(x_{j}(t) x\right)=\Delta^{\omega_{i}}(x)$ now follows from (2.15).

Our next proposition justifies the validity of Definition 1.4.

Proposition 2.3. For any $x \in G$ and any $j \neq i$, we have

$$
\Delta^{\omega_{i}}\left(x \overline{s_{j}}\right)=\Delta^{\omega_{i}}\left(\overline{\overline{s_{j}}} x\right)=\Delta^{\omega_{i}}(x) .
$$

Proof. Follows from (2.14), (2.16), and (1.8).

The extension of principal minors from the open subset $G_{0}$ to the whole of $G$ is given as follows. The Bruhat decomposition theorem implies that every $x \in G$ can be written as

$$
x=x^{-} a \bar{w} x^{+},
$$

for some $x^{-} \in N_{-}, a \in H, w \in W$, and $x^{+} \in N$; moreover, the elements $a \in H$ and $w \in W$ are uniquely determined by $x$. 
Proposition 2.4. If $x \in G$ is expressed in the form (2.18), then

$$
\Delta^{\omega_{i}}(x)= \begin{cases}a^{\omega_{i}} & \text { if } w \omega_{i}=\omega_{i} \\ 0, & \text { otherwise }\end{cases}
$$

Proof. By (2.14), we have

$$
\Delta^{\omega_{i}}\left(x^{-} a \bar{w} x^{+}\right)=a^{\omega_{i}} \Delta^{\omega_{i}}(\bar{w}) .
$$

Thus, to prove (2.19) we only need to show that

$$
\Delta^{\omega_{i}}(\bar{w})= \begin{cases}1 & \text { if } w \omega_{i}=\omega_{i} \\ 0, & \text { otherwise }\end{cases}
$$

The formula is obvious for $w=e$, the identity element of $W$. Hence we can assume that $\ell(w) \geq 1$ and write $w$ as $u s_{j}$ for some $u \in W$ and $j \in[1, r]$ with $\ell(u)=\ell(w)-1$. Since $\Delta^{\omega_{i}}$ is a regular function on $G$, we have

$$
\Delta^{\omega_{i}}(\bar{w})=\Delta^{\omega_{i}}\left(\bar{u} \overline{s_{j}}\right)=\lim _{t \rightarrow 0} \Delta^{\omega_{i}}\left(\bar{u} x_{j}(t) \overline{s_{j}}\right) .
$$

Substituting into (2.21) the expression for $x_{j}(t) \overline{s_{j}}$ given by (2.13) and using (2.14), we obtain

$$
\Delta^{\omega_{i}}(\bar{w})=\lim _{t \rightarrow 0} t^{\omega_{i}\left(h_{j}\right)} \Delta^{\omega_{i}}\left(\bar{u} x_{\bar{j}}\left(t^{-1}\right)\right) .
$$

Since $\ell(u)=\ell(w)-1$, the root $u\left(\alpha_{j}\right)$ is positive, implying that $\bar{u} x_{\bar{j}}\left(t^{-1}\right) \bar{u}^{-1} \in N_{-}$. Again using (2.14), we obtain

$$
\Delta^{\omega_{i}}(\bar{w})=\lim _{t \rightarrow 0} t^{\delta_{i j}} \Delta^{\omega_{i}}(\bar{u})
$$

It follows that

$$
\Delta^{\omega_{i}}(\bar{w})= \begin{cases}\Delta^{\omega_{i}}(\bar{u}) & \text { if } j \neq i \\ 0, & \text { otherwise }\end{cases}
$$

This implies (2.20) by induction on $\ell(w)$.

As a corollary, we obtain the following useful characterization of the set $G_{0}$.

Corollary 2.5. An element $x \in G$ has Gaussian decomposition if and only if $\Delta^{\omega_{i}}(x) \neq 0$ for any $i \in[1, r]$.

In subsequent proofs, we will also make use of the following identities.

Proposition 2.6. For any $x=[x]_{-}[x]_{0}[x]_{+} \in G_{0}$ and any $w \in W$, we have

$$
\begin{aligned}
& \Delta_{\omega_{i}, w \omega_{i}}\left([x]_{+}\right)=\frac{\Delta_{\omega_{i}, w \omega_{i}}(x)}{\Delta^{\omega_{i}}(x)}, \\
& \Delta_{w \omega_{i}, \omega_{i}}\left([x]_{-}\right)=\frac{\Delta_{w \omega_{i}, \omega_{i}}(x)}{\Delta^{\omega_{i}}(x)} .
\end{aligned}
$$

Proof. Using (1.10) and (2.14), we obtain:

$$
\Delta_{\omega_{i}, w \omega_{i}}(x)=\Delta^{\omega_{i}}(x \bar{w})=[x]_{0}^{\omega_{i}} \Delta^{\omega_{i}}\left([x]_{+} \bar{w}\right)=\Delta^{\omega_{i}}(x) \Delta_{\omega_{i}, w \omega_{i}}\left([x]_{+}\right),
$$

which proves (2.22). The proof of (2.23) is similar.

The transformation $\left(-w_{\mathrm{o}}\right)$ permutes fundamental weights. We will use the notation $i \mapsto i^{*}$ for the induced permutation of the index set $[1, r]$, so that

$$
\omega_{i^{*}}=-w_{\mathrm{o}}\left(\omega_{i}\right)(i \in[1, r]) .
$$


Proposition 2.7. For any $x \in G$ and any $u, v \in W$, we have

$$
\Delta_{u \omega_{i}, v \omega_{i}}(x)=\Delta_{v \omega_{i}, u \omega_{i}}\left(x^{T}\right)=\Delta_{v w_{\mathrm{o}} \omega_{i^{*}}, u w_{\mathrm{o}} \omega_{i^{*}}}\left(x^{\iota}\right) .
$$

Proof. Using (2.15) and (2.4), we obtain:

$$
\Delta_{u \omega_{i}, v \omega_{i}}(x)=\Delta^{\omega_{i}}\left(\overline{\overline{u^{-1}}} x \bar{v}\right)=\Delta^{\omega_{i}}\left(\left(\overline{\overline{u^{-1}}} x \bar{v}\right)^{T}\right)=\Delta^{\omega_{i}}\left(\overline{\overline{v^{-1}}} x^{T} \bar{u}\right)=\Delta_{v \omega_{i}, u \omega_{i}}\left(x^{T}\right),
$$

which proves the first equality in (2.25).

To prove the second equality, let us introduce the anti-automorphism $\eta$ of $G$ by $\eta(x)=\overline{\overline{w_{\mathrm{o}}}} x^{\iota} \overline{w_{\mathrm{o}}}$. Since $\eta$ preserves $H$ and interchanges $N_{-}$and $N$, it follows that $\eta$ preserves $G_{0}$, and $[\eta(x)]_{0}=\eta\left([x]_{0}\right)=\overline{\overline{w_{\mathrm{o}}}}[x]_{0}^{-1} \overline{w_{\mathrm{o}}}$ for any $x \in G_{0}$. Using (1.2) and (2.24), we conclude that $\Delta^{\omega_{i}}(\eta(x))=\Delta^{\omega_{i *}}(x)$. Hence

$$
\begin{aligned}
\Delta_{u \omega_{i}, v \omega_{i}}(x) & =\Delta^{\omega_{i}}(\overline{\overline{\overline{u-1}}} x \bar{v})=\Delta^{\omega_{i^{*}}}\left(\eta\left(\overline{\overline{u^{-1}}} x \bar{v}\right)\right)=\Delta^{\omega_{i^{*}}}\left(\overline{\overline{w_{\mathrm{o}}}} \overline{\bar{v}}^{-1} x^{\iota} \overline{u^{-1}}{ }^{-1} \overline{w_{\mathrm{o}}}\right) \\
& =\Delta^{\omega_{i^{*}}}\left(\overline{\overline{w_{\mathrm{o}} v^{-1}}} x^{\iota} \overline{u w_{\mathrm{o}}}\right)=\Delta_{v w_{\mathrm{o}} \omega_{i^{*}}, u w_{\mathrm{o}} \omega_{i^{*}}}\left(x^{\iota}\right)
\end{aligned}
$$

as claimed.

Proof of Theorem 1.17. First of all, since $\overline{\overline{s_{i} u^{-1}}}=\overline{\overline{s_{i}}} \overline{\overline{u^{-1}}}$ and $\overline{v s_{i}}=\bar{v} \overline{s_{i}}$, the definition (1.10) implies that it is enough to prove (1.25) in the case when $u=v=e$, the identity element. Thus, we only need to show that

$$
\Delta_{\omega_{i}, \omega_{i}} \Delta_{s_{i} \omega_{i}, s_{i} \omega_{i}}-\Delta_{s_{i} \omega_{i}, \omega_{i}} \Delta_{\omega_{i}, s_{i} \omega_{i}}=\prod_{j \neq i} \Delta_{\omega_{j}, \omega_{j}}^{-a_{j i}} .
$$

As in the case of Proposition 2.2, our proof of (2.26) will rely on representation theory. Consider the representation $\rho$ of the group $G \times G$ in $\mathbb{C}[G]$ given by

$$
\rho\left(x_{1}, x_{2}\right) f(x)=f\left(x_{1}^{T} x x_{2}\right) .
$$

Let us denote the left- and right-hand sides of (2.26) by $f_{1}$ and $f_{2}$, respectively. We first verify that the function $f_{2} \in \mathbb{C}[G]$ has the following properties:

(1) $f_{2}$ is a highest weight vector in the representation $\rho$, i.e., it is invariant under the subgroup $N \times N \subset G \times G$;

(2) $f_{2}$ has weight $(\gamma, \gamma)$, where $\gamma=2 \omega_{i}-\alpha_{i}$; that is, $\rho\left(a_{1}, a_{2}\right) f_{2}=\left(a_{1} a_{2}\right)^{\gamma} f_{2}$ for any $a_{1}, a_{2} \in H$

(3) $f_{2}(e)=1$ (here $e$ stands for the identity element of $G$ ).

Property (3) is trivial, while (1) follows from (2.14). Also by (2.14), $f_{2}$ has weight $\left(-\sum_{j \neq i} a_{j i} \omega_{j},-\sum_{j \neq i} a_{j i} \omega_{j}\right)$. To prove (2), it is enough to show that $-\sum_{j \neq i} a_{j i} \omega_{j}=\gamma$; but this follows from the equality

$$
\sum_{j \in[1, r]} a_{j i} \omega_{j}=\alpha_{i}
$$

which can be taken as a definition of the Cartan matrix.

Properties (1)-(3) uniquely determine the restriction of $f_{2}$ to $G_{0}$. Since $G_{0}$ is dense in $G$, and $f_{2}$ is regular, these properties uniquely determine $f_{2}$. It remains to show that $f_{1}$ satisfies (1)-(3).

The normalization condition (3) follows from Proposition 2.4; indeed, in view of $(2.19)$

$$
\Delta_{\omega_{i}, \omega_{i}}(e)=\Delta_{s_{i} \omega_{i}, s_{i} \omega_{i}}(e)=1, \Delta_{s_{i} \omega_{i}, \omega_{i}}(e)=\Delta_{\omega_{i}, s_{i} \omega_{i}}(e)=0
$$


To prove that $f_{1}$ satisfies (2), notice that for any $u, v \in W$, the function $\Delta_{u \omega_{i}, v \omega_{i}}$ has weight $\left(u \omega_{i}, v \omega_{i}\right)$ (this follows from (1.10) and (1.2)). Hence both summands in $f_{1}$ have weight $\left(\omega_{i}+s_{i} \omega_{i}, \omega_{i}+s_{i} \omega_{i}\right)=(\gamma, \gamma)$.

To prove that $f_{1}$ satisfies (1), we first notice that, in view of (2.25), we have $f_{1}\left(x^{T}\right)=f_{1}(x)$ for any $x \in G$, hence $\rho\left(x_{1}, x_{2}\right) f_{1}=\rho\left(x_{2}, x_{1}\right) f_{1}$ for any $x_{1}, x_{2} \in G$. Therefore, it suffices to show that $f_{1}$ is invariant under the action of $N$ by right translations. Let $E_{1}, \ldots, E_{r}$ be the infinitesimal right translation operators on $\mathbb{C}[G]$ defined by

$$
E_{j}(f)(x)=\left.\frac{d}{d t} f\left(x x_{j}(t)\right)\right|_{t=0} ;
$$

each $E_{j}$ is a derivation of the ring $\mathbb{C}[G]$. It is enough to show that $E_{j} f_{1}=0$ for all $j$. If $j \neq i$, then $E_{j}$ annihilates all four minors that appear in $f_{1}$ (this follows from the fact that $\left.\overline{s_{i}} x_{j}(t){\overline{s_{i}}}^{-1} \in N\right)$, hence $E_{j} f_{1}=0$. It remains to prove that $E_{i} f_{1}=0$. Clearly, we have

$$
E_{i} \Delta_{\omega_{i}, \omega_{i}}=E_{i} \Delta_{s_{i} \omega_{i}, \omega_{i}}=0 .
$$

We claim that

$$
E_{i} \Delta_{\omega_{i}, s_{i} \omega_{i}}=\Delta_{\omega_{i}, \omega_{i}}, E_{i} \Delta_{s_{i} \omega_{i}, s_{i} \omega_{i}}=\Delta_{s_{i} \omega_{i}, \omega_{i}} .
$$

Combining (2.28) and (2.29) and using the Leibniz rule, we obtain

$$
\begin{aligned}
E_{i} f_{1} & =E_{i}\left(\Delta_{\omega_{i}, \omega_{i}} \Delta_{s_{i} \omega_{i}, s_{i} \omega_{i}}-\Delta_{s_{i} \omega_{i}, \omega_{i}} \Delta_{\omega_{i}, s_{i} \omega_{i}}\right) \\
& =\Delta_{\omega_{i}, \omega_{i}} \Delta_{s_{i} \omega_{i}, \omega_{i}}-\Delta_{\omega_{i}, \omega_{i}} \Delta_{s_{i} \omega_{i}, \omega_{i}}=0,
\end{aligned}
$$

as required. We will deduce (2.29) from the following lemma which is a standard fact in the representation theory of $S L_{2}$.

Lemma 2.8. Suppose $f \in \mathbb{C}[G]$ is such that $E_{i} f=0$ and $f\left(x t^{h_{i}}\right)=t^{k} f(x)$ for some $k \geq 0$. Let $f^{\prime} \in \mathbb{C}[G]$ be given by $f^{\prime}(x)=f\left(x \overline{s_{i}}\right)$. Then $E_{i}^{k}\left(f^{\prime}\right)=k ! f$.

The first equality in (2.29) follows by applying this lemma to $f=\Delta_{\omega_{i}, \omega_{i}}$ (in this case, $k=1$ and $f^{\prime}=\Delta_{\omega_{i}, s_{i} \omega_{i}}$ ). Similarly, the second equality in (2.29) follows by applying Lemma 2.8 to $f=\Delta_{s_{i} \omega_{i}, \omega_{i}}$ (in this case, $k=1$ and $f^{\prime}=\Delta_{s_{i} \omega_{i}, s_{i} \omega_{i}}$ ). This completes the proof of Theorem 1.17.

2.4. Affine coordinates in Schubert cells. For every $w \in W$, the corresponding Schubert cell $(B w B) / B \subset G / B$ is the image of the Bruhat cell $B w B$ under the natural projection of $G$ onto the flag variety $G / B$.

Let the subgroups $N_{+}(w) \subset N$ and $N_{-}(w) \subset N_{-}$be defined by

$$
N_{+}(w)=N \cap \tilde{w} N_{-} \tilde{w}^{-1}, \quad N_{-}(w)=N_{-} \cap \tilde{w}^{-1} N \tilde{w},
$$

where $\tilde{w}$ is any representative of $w$ in $G$; since $H$ normalizes $N$ and $N_{-}$, these subgroups do not depend on the choice of $\tilde{w}$. The following proposition is essentially well known (cf. [11, Corollary 23.60]).

Proposition 2.9. An element $x \in G$ lies in the Bruhat cell $B w B$ if and only if, for some (equivalently, any) representative $\tilde{w} \in G$ of $w$, we have $\tilde{w}^{-1} x \in G_{0}$ and $\left[\tilde{w}^{-1} x\right]_{-} \in N_{-}(w)$. Furthermore, the element

$$
y_{+}=\pi_{+}(x)=\tilde{w}\left[\tilde{w}^{-1} x\right]_{-} \tilde{w}^{-1} \in N_{+}(w)
$$

does not depend on the choice of $\tilde{w}$, and the correspondence $\pi_{+}: x \mapsto y_{+}$induces a biregular isomorphism between the Schubert cell $(B w B) / B$ and $N_{+}(w)$. 
Using the transpose map $x \mapsto x^{T}$, one obtains a counterpart of Proposition 2.9 for the opposite Bruhat cell $B_{-} w B_{-}$.

Proposition 2.10. An element $x \in G$ lies in $B_{-} w B_{-}$if and only if, for some (equivalently, any) representative $\tilde{w} \in G$ of $w$, we have $x \tilde{w}^{-1} \in G_{0}$ and $\left[x \tilde{w}^{-1}\right]_{+} \in$ $N_{+}(w)$. Furthermore, the element

$$
y_{-}=\pi_{-}(x)=\tilde{w}^{-1}\left[x \tilde{w}^{-1}\right]_{+} \tilde{w} \in N_{-}(w)
$$

does not depend on the choice of $\tilde{w}$, and the correspondence $\pi_{-}: x \mapsto y_{-}$induces a biregular isomorphism between the "opposite Schubert cell" $B_{-} \backslash\left(B_{-} w B_{-}\right)$and $N_{-}(w)$.

The group $N_{-}(w)$ is a unipotent Lie group of dimension $\ell=\ell(w)$, hence it is isomorphic to the affine space $\mathbb{C}^{\ell}$ as an algebraic variety. We will associate with any $\mathbf{i}=\left(i_{1}, \ldots, i_{\ell}\right) \in R(w)$ the following system of affine coordinates on $N_{-}(w)$. For $\left(p_{1}, \ldots, p_{\ell}\right) \in \mathbb{C}^{\ell}$, we set

$$
y_{\mathbf{i}}\left(p_{1}, \ldots, p_{\ell}\right)=\overline{\bar{w}}^{-1} \cdot \overline{\overline{s_{i_{1}}}} x_{\overline{i_{1}}}\left(p_{1}\right) \ldots \overline{\overline{s_{\ell}}} x_{\overline{i_{\ell}}}\left(p_{\ell}\right) .
$$

Also, let us define

$$
w_{k}=w_{k, \mathbf{i}}=s_{i_{\ell}} s_{i_{\ell-1}} \cdots s_{i_{k}}
$$

for $k \in[1, \ell+1]$, so that $w_{1}=w^{-1}$ and $w_{\ell+1}=e$.

Proposition 2.11. The map $\left(p_{1}, \ldots, p_{\ell}\right) \mapsto y=y_{\mathbf{i}}\left(p_{1}, \ldots, p_{\ell}\right)$ is a biregular isomorphism between $\mathbb{C}^{\ell}$ and $N_{-}(w)$. The inverse map is given by

$$
p_{k}=\Delta_{w_{k} \omega_{i_{k}}, w_{k+1} \omega_{i_{k}}}(y) \text {. }
$$

Proof. We can rewrite (2.33) as

$$
y_{\mathbf{i}}\left(p_{1}, \ldots, p_{\ell}\right)=\prod_{k=1}^{\ell} \overline{w_{k+1}} x_{\overline{i_{k}}}\left(p_{k}\right) \overline{w_{k+1}}-1 .
$$

Each factor $\overline{w_{k+1}} x_{\overline{i_{k}}}\left(p_{k}\right){\overline{w_{k+1}}}^{-1}$ belongs to the root subgroup in $G$ corresponding to the root $-w_{k+1}\left(\alpha_{i_{k}}\right)$, and these are all the root subgroups in $N_{-}(w)$ (cf. [5, VI, 1.6]). This implies the first statement in Proposition 2.11. To prove (2.35), we set $\mathbf{i}^{\prime}=\left(i_{1}, \ldots, i_{k-1}\right)$ and $\mathbf{i}^{\prime \prime}=\left(i_{k+1}, \ldots, i_{\ell}\right)$ so that $\mathbf{i}=\left(\mathbf{i}^{\prime}, i_{k}, \mathbf{i}^{\prime \prime}\right)$. Let $y^{\prime}=$ $y_{\mathbf{i}^{\prime}}\left(p_{1}, \ldots, p_{k-1}\right)$ and $y^{\prime \prime}=y_{\mathbf{i}^{\prime \prime}}\left(p_{k+1}, \ldots, p_{\ell}\right)$. In view of $(2.33)$, we have

$$
\overline{\overline{w_{k}^{-1}}} y \overline{w_{k+1}}=y^{\prime}\left(\overline{\overline{s_{i_{k}}}} x_{\overline{i_{k}}}\left(p_{k}\right)\right)\left(\overline{\overline{w_{k+1}^{-1}}} y^{\prime \prime} \overline{w_{k+1}}\right) \text {. }
$$

In this decomposition, the first factor $y^{\prime}$ belongs to $N_{-}\left(s_{i_{1}} \cdots s_{i_{k-1}}\right) \subset N_{-}$, while the last factor $\overline{\overline{w_{k+1}^{-1}}} y^{\prime \prime} \overline{w_{k+1}}$ belongs to $w_{k+1}^{-1} N_{-}\left(w_{k+1}^{-1}\right) w_{k+1} \subset N$. Using (2.14) and (2.13), we conclude that

$$
\begin{aligned}
& \Delta_{w_{k} \omega_{i_{k}}, w_{k+1} \omega_{i_{k}}}(y)=\Delta^{\omega_{i_{k}}}\left(\overline{\overline{w_{k}^{-1}}} y \overline{\overline{w_{k+1}}}\right)=\Delta^{\omega_{i_{k}}}\left(\overline{\overline{s_{i_{k}}}} x_{\overline{i_{k}}}\left(p_{k}\right)\right) \\
& =\Delta^{\omega_{i_{k}}}\left(x_{\overline{i_{k}}}\left(-p_{k}^{-1}\right) p_{k}^{h_{i_{k}}} x_{i_{k}}\left(p_{k}^{-1}\right)\right)=p_{k}
\end{aligned}
$$

as claimed.

Note for future use that a similar argument allows us to prove that, for any $i \in[1, r], k \in[1, \ell+1]$, and $y \in N_{-}(w)$, we have

$$
\Delta_{w_{k} \omega_{i}, w_{k} \omega_{i}}(y)=1 .
$$


This follows from a decomposition similar to (2.36):

$$
{\overline{w_{k}}}^{-1} y \overline{w_{k}}=y^{\prime}\left({\overline{w_{k}}}^{-1} y^{\prime \prime} \overline{w_{k}}\right) \text {, }
$$

where $y^{\prime} \in N_{-}\left(s_{i_{1}} \cdots s_{i_{k-1}}\right) \subset N_{-}$, and $\overline{w_{k}}-1 y^{\prime \prime} \overline{w_{k}} \in w_{k}^{-1} N_{-}\left(w_{k}^{-1}\right) w_{k} \subset N$.

As a corollary of Proposition 2.11, we obtain defining equations for $N_{-}(w)$ as a subvariety in $N_{-}$. Notice that $N_{-}=N_{-}\left(w_{\mathrm{o}}\right)$. Hence, for every $\mathbf{j}=\left(j_{1}, \ldots, j_{n}\right) \in$ $R\left(w_{\mathrm{o}}\right)$, any element $y \in N_{-}$can be uniquely written as $y=y_{\mathbf{j}}\left(p_{1}, \ldots, p_{n}\right)$ for some $\left(p_{1}, \ldots, p_{n}\right) \in \mathbb{C}^{n}$ (here $\left.n=\ell\left(w_{\mathrm{o}}\right)\right)$. Let us choose $\mathbf{j}$ so that its first $n-\ell$ indices form a reduced word $\mathbf{j}_{1}$ for $w_{\mathrm{o}} w^{-1}$, while the last $\ell$ indices form a reduced word $\mathbf{j}_{2}$ for $w$. Then write $\left(w_{\mathrm{o}}\right)_{k}=s_{j_{n}} s_{j_{n-1}} \cdots s_{j_{k}}$ for $k \in[1, n+1]$, in agreement with (2.34). Finally, let us denote

$$
N_{-}^{\prime}(w)=N_{-} \cap \tilde{w}^{-1} N_{-} \tilde{w},
$$

where $\tilde{w}$ is any representative of $w$ in $G$ (cf. (2.30)). The following proposition is an immediate consequence of Proposition 2.11 and the definition (2.33).

Proposition 2.12. Every $y \in N_{-}$is uniquely written as $y=y_{1} y_{2}$ with $y_{1} \in N_{-}^{\prime}(w)$ and $y_{2} \in N_{-}(w)$. In the above notation, if $y=y_{\mathbf{j}}\left(p_{1}, \ldots, p_{n}\right)$, then

$$
y_{1}=\overline{\bar{w}}^{-1} y_{\mathbf{j}_{1}}\left(p_{1}, \ldots, p_{n-\ell}\right) \overline{\bar{w}}, y_{2}=y_{\mathbf{j}_{2}}\left(p_{n-\ell+1}, \ldots, p_{n}\right) .
$$

Hence $y$ lies in $N_{-}(w)$ if and only if

$$
\Delta_{\left(w_{\circ}\right)_{k} \omega_{j_{k}},\left(w_{\circ}\right)_{k+1} \omega_{j_{k}}}(y)=0
$$

for $k=1, \ldots, n-\ell$.

2.5. $y$-coordinates in double Bruhat cells. Let us fix a pair $(u, v) \in W \times W$ and consider the open subset $G_{0}^{u, v}=G^{u, v} \cap G_{0}$ consisting of the elements $x$ in the double Bruhat cell $G^{u, v}$ that admit Gaussian decomposition (1.5). In view of Propositions 2.9 and 2.10, the restrictions $\pi_{+}: G^{u, v} \rightarrow N_{+}(u)$ and $\pi_{-}: G^{u, v} \rightarrow$ $N_{-}(v)$ are well defined. Let us also introduce the map $\pi_{0}: G_{0}^{u, v} \rightarrow H$ by

$$
y_{0}=\pi_{0}(x)=[x]_{0},
$$

thus obtaining the map

$$
\pi^{u, v}=\left(\pi_{+}, \pi_{0}, \pi_{-}\right): G_{0}^{u, v} \rightarrow N_{+}(u) \times H \times N_{-}(v) .
$$

For $x \in G_{0}^{u, v}$, we will write $\pi^{u, v}(x)=\left(y_{+}, y_{0}, y_{-}\right)$and call this triple the $y$ coordinates of $x$.

Example 2.13. Let $G=S L_{2}(\mathbb{C})$, and let $u=v=w_{\mathrm{o}}$ (cf. Example 1.8). A matrix $x=\left(\begin{array}{ll}x_{11} & x_{12} \\ x_{21} & x_{22}\end{array}\right)$ with determinant 1 belongs to $G_{0}^{u, v}$ if and only if $x_{11} \neq 0$, $x_{12} \neq 0, x_{21} \neq 0$. Using formulas (2.31), (2.32), and (2.39), we see that the $y$-coordinates of $x$ are given by

$$
y_{+}=\left(\begin{array}{cc}
1 & x_{11} x_{21}^{-1} \\
0 & 1
\end{array}\right), \quad y_{0}=\left(\begin{array}{cc}
x_{11} & 0 \\
0 & x_{11}^{-1}
\end{array}\right), \quad y_{-}=\left(\begin{array}{cc}
1 & 0 \\
x_{11} x_{12}^{-1} & 1
\end{array}\right) \text {. }
$$

Our use of the term "coordinates" for the triple $\left(y_{+}, y_{0}, y_{-}\right)$is justified by the following statement. 
Proposition 2.14. The map $\pi^{u, v}$ is a biregular isomorphism

$$
G_{0}^{u, v} \rightarrow\left(N_{+}(u) \cap G_{0} u^{-1}\right) \times H \times\left(N_{-}(v) \cap v^{-1} G_{0}\right) .
$$

The inverse isomorphism $\left(y_{+}, y_{0}, y_{-}\right) \mapsto x$ is given by

$$
[x]_{-}=\left[y_{+} \tilde{u}\right]_{-}, \quad[x]_{0}=y_{0},[x]_{+}=\left[\tilde{v} y_{-}\right]_{+},
$$

where $\tilde{u}$ and $\tilde{v}$ are arbitrary representatives of $u$ and $v$.

Proof. By Proposition 2.9, any $x \in B u B$ can be written as $x=y_{+} \tilde{u} b$, where $y_{+}=\pi_{+}(x)$ and $b \in B$. It follows that $x \in G_{0}$ if and only if $y_{+} \tilde{u} \in G_{0}$, and if this is the case, then $[x]_{-}=\left[y_{+} \tilde{u}\right]_{-}$. Similarly, an element $x \in B_{-} v B_{-}$lies in $G_{0}$ if and only if $\tilde{v} y_{-} \in G_{0}$, and then $[x]_{+}=\left[\tilde{v} y_{-}\right]_{+}$. It follows that $\pi^{u, v}$ is an embedding of $G_{0}^{u, v}$ into $\left(N_{+}(u) \cap G_{0} u^{-1}\right) \times H \times\left(N_{-}(v) \cap v^{-1} G_{0}\right)$, and that the inverse map is given by (2.41). The same argument shows that if the triple $\left(y_{+}, y_{0}, y_{-}\right)$lies in $\left(N_{+}(u) \cap G_{0} u^{-1}\right) \times H \times\left(N_{-}(v) \cap v^{-1} G_{0}\right)$, then the element $x$ given by (2.41) lies in $G_{0}^{u, v}$, and we are done.

The following proposition is immediate from the definitions.

Proposition 2.15. We have $N_{+}(u)^{T}=N_{-}\left(u^{-1}\right)$ and $\left(G_{0}^{u, v}\right)^{T}=G_{0}^{v^{-1}, u^{-1}}$. If $x \in G_{0}^{u, v}$ has $y$-coordinates $\left(y_{+}, y_{0}, y_{-}\right)$, then $x^{T}$ has $y$-coordinates $\left(y_{-}^{T}, y_{0}, y_{+}^{T}\right)$.

This proposition shows that the transpose map "interchanges" the coordinates $y_{+}$and $y_{-}$, so that any statement about $y_{-}$has a counterpart for $y_{+}$. For instance, Proposition 2.9 is a counterpart of Proposition 2.10 in this sense.

Proposition 2.16. Suppose $x \in G_{0}^{u, v}$ has the $y$-coordinates $\left(y_{+}, y_{0}, y_{-}\right)$. Then

$$
\left[\overline{\overline{u^{-1}}} x\right]_{0}^{-1}=\left[y_{+} \bar{u}\right]_{0} y_{0}^{-1},\left[x \overline{v^{-1}}\right]_{0}^{-1}=y_{0}^{-1}\left[\overline{\bar{v}} y_{-}\right]_{0} .
$$

Proof. By Proposition 2.9, $x=y_{+} \bar{u} b$ for some $b \in B$. It follows that $y_{0}=\left[y_{+} \bar{u}\right]_{0}[b]_{0}$. On the other hand, $\overline{\overline{u^{-1}}} x=\bar{u}^{-1} y_{+} \bar{u} b \in N_{-} b$, hence

$$
\left[\overline{\overline{u^{-1}}} x\right]_{0}=[b]_{0}=y_{0}\left[y_{+} \bar{u}\right]_{0}^{-1} .
$$

This proves the first equality in (2.42); the second one follows by Proposition 2.15.

It will be of special importance for us to specialize Proposition 2.14 to the case when $(u, v)=(e, w)$, where $e$ is the identity element of $W$, and $w \in W$ is arbitrary. Then we have $G_{0}^{e, w}=G^{e, w}=H N^{w}$ where

$$
N^{w}=N \cap B_{-} w B_{-} .
$$

Specializing Proposition 2.14 to this case, we obtain the following statement.

Proposition 2.17. For any $w \in W$, the map $\pi_{-}: B_{-} w B_{-} \rightarrow N_{-}(w)$ restricts to a biregular isomorphism $N^{w} \rightarrow N_{-}(w) \cap w^{-1} G_{0}$. The inverse isomorphism $N_{-}(w) \cap$ $w^{-1} G_{0} \rightarrow N^{w}$ is given by $y \mapsto[\tilde{w} y]_{+}$, where $\tilde{w}$ is an arbitrary representative of $w$.

Using Proposition 2.15, we see that Proposition 2.14 is equivalent to its special case given by Proposition 2.17 combined with the following decomposition:

$$
G_{0}^{u, v}=\left(N^{u^{-1}}\right)^{T} H N^{v} .
$$


2.6. Factorization problem in Schubert cells. In this section we recall some results from [4] concerning a version of the factorization problem for the variety $N^{w}=N \cap B_{-} w B_{-}$(cf. (2.43)). We will need the following analogue of Theorem 1.2 which is essentially due to G. Lusztig [18] (cf. also [4, Proposition 1.1]).

Proposition 2.18. For any $w \in W$ and any reduced word $\mathbf{i}=\left(i_{1}, \ldots, i_{\ell}\right) \in R(w)$, the map $\left(t_{1}, \ldots, t_{\ell}\right) \mapsto x_{i_{1}}\left(t_{1}\right) \cdots x_{i_{\ell}}\left(t_{\ell}\right)$ is a biregular isomorphism between $\mathbb{C}_{\neq 0}^{\ell}$ and a Zariski open subset of $N^{w}$.

We will give explicit formulas for the inverse of the product map in Proposition 2.18 .

Theorem 2.19. Let $\mathbf{i}=\left(i_{1}, \ldots, i_{\ell}\right) \in R(w)$, and let $x=x_{i_{1}}\left(t_{1}\right) \cdots x_{i_{\ell}}\left(t_{\ell}\right) \in N^{w}$ with all $t_{k}$ nonzero complex numbers. Then the $t_{k}$ are recovered from $x$ by

$$
t_{k}=\frac{1}{\Delta_{w_{k} \omega_{i_{k}}, \omega_{i_{k}}}(y) \Delta_{w_{k+1} \omega_{i_{k}}, \omega_{i_{k}}}(y)} \prod_{j \neq i_{k}} \Delta_{w_{k+1} \omega_{j}, \omega_{j}}(y)^{-a_{j, i_{k}}},
$$

where $w_{k}$ is given by (2.34), and $y=\pi_{-}(x) \in N_{-}(w)$ (cf. (2.32)).

This theorem is a reformulation of $[4$, Theorems 1.4, 6.2]. Here we present a new proof which is in some sense more elementary than the one in [4], and also provides additional information that we will need later on.

Proof. There is nothing to prove if $w=e$, so we will assume that $\ell(w)=\ell \geq 1$. Let $y=\pi_{-}(x)$ and $z=\overline{\bar{w}} y$. By Proposition 2.17, $z \in G_{0}$ and $x=[z]_{+}$. Let us write $i_{1}=i$, and denote $w^{\prime}=s_{i} w, \mathbf{i}^{\prime}=\left(i_{2}, \ldots, i_{\ell}\right) \in R\left(w^{\prime}\right), x^{\prime}=x_{i}\left(-t_{1}\right) x=$ $x_{i_{2}}\left(t_{2}\right) \cdots x_{i_{\ell}}\left(t_{\ell}\right) \in N^{w^{\prime}}, y^{\prime}=\pi_{-}\left(x^{\prime}\right) \in N_{-}\left(w^{\prime}\right)$, and $z^{\prime}=\overline{\overline{w^{\prime}}} y^{\prime}$. Here is the key lemma.

Lemma 2.20. In the notation just introduced, let us write $y^{\prime}=y_{\mathbf{i}^{\prime}}\left(p_{2}, \ldots, p_{\ell}\right)$, in accordance with Proposition 2.11. Then $y=y_{\mathbf{i}}\left(p_{1}, p_{2}, \ldots, p_{\ell}\right)$, where $p_{1}$ is given by

$$
p_{1}=\Delta_{s_{i} \omega_{i}, \omega_{i}}\left(x_{\bar{i}}\left(\left[z^{\prime}\right]_{0}^{-\alpha_{i}} t_{1}^{-1}\right)\left[z^{\prime}\right]_{-}^{-1}\right) .
$$

Furthermore, we have

$$
t_{1}=\left[z^{\prime}\right]_{0}^{\omega_{i}-\alpha_{i}}[z]_{0}^{-\omega_{i}} .
$$

Proof. Let us temporarily denote $\tilde{y}=y_{\mathbf{i}}\left(p_{1}, p_{2}, \ldots, p_{\ell}\right)$ and $\tilde{z}=\overline{\bar{w}} \tilde{y}$, where $p_{1}$ is given by (2.46); our goal is to show that $\tilde{y}=y$ and $\tilde{z}=z$. By Proposition 2.17, it suffices to show that $[\tilde{z}]_{+}=x$, or equivalently that $\tilde{z} x^{-1} \in B_{-}$.

By Proposition 2.12 (applied to $w=s_{i}$ ), formula (2.46) implies that

$$
x_{\bar{i}}\left(\left[z^{\prime}\right]_{0}^{-\alpha_{i}} t_{1}^{-1}\right)\left[z^{\prime}\right]_{-}^{-1}=\overline{s_{i}} y^{\prime \prime}{\overline{s_{i}}}^{-1} x_{\bar{i}}\left(p_{1}\right)
$$

where $y^{\prime \prime} \in N_{-}$. Using (2.5) and (2.12), we can rewrite the left-hand side of (2.48) as follows:

$$
\begin{aligned}
x_{\bar{i}}\left(\left[z^{\prime}\right]_{0}^{-\alpha_{i}} t_{1}^{-1}\right)\left[z^{\prime}\right]_{-}^{-1} & =\left[z^{\prime}\right]_{0} x_{\bar{i}}\left(t_{1}^{-1}\right)\left[z^{\prime}\right]_{0}^{-1}\left[z^{\prime}\right]_{-}^{-1}=\left[z^{\prime}\right]_{0} x_{\bar{i}}\left(t_{1}^{-1}\right) x^{\prime}\left(z^{\prime}\right)^{-1} \\
& =\left[z^{\prime}\right]_{0} x_{\bar{i}}\left(t_{1}^{-1}\right) x_{i}\left(-t_{1}\right) x\left(z^{\prime}\right)^{-1}=\left[z^{\prime}\right]_{0} \overline{s_{i}} t_{1}^{-h_{i}} x_{\bar{i}}\left(-t_{1}^{-1}\right) x\left(z^{\prime}\right)^{-1} .
\end{aligned}
$$

Substituting this expression into (2.48) and using the fact that $\tilde{z}={\overline{s_{i}}}^{-1} x_{\bar{i}}\left(p_{1}\right) z^{\prime}$, we can rewrite (2.48) as follows:

$$
\tilde{z} x^{-1}=\left(y^{\prime \prime}\right)^{-1}{\overline{s_{i}}}^{-1}\left[z^{\prime}\right]_{0}{\overline{s_{i}}}_{1} t_{1}^{-h_{i}} x_{\bar{i}}\left(-t_{1}^{-1}\right) .
$$


It follows that $\tilde{z} x^{-1} \in B_{-}$, hence $\tilde{y}=y$ and $\tilde{z}=z$. Since the left-hand side of (2.49) is equal to $[z]_{-}[z]_{0}$, it follows that

$$
[z]_{0}={\overline{s_{i}}}^{-1}\left[z^{\prime}\right]_{0} \overline{s_{i}} t_{1}^{-h_{i}} .
$$

Finally, (2.47) follows from (2.50) by applying the character $a \mapsto a^{\omega_{i}}$ to both sides and using (1.2).

Note that (2.46) can be simplified as follows:

$$
p_{1}=\Delta_{\omega_{i}, w^{\prime-1} \omega_{i}}\left(x y^{\prime-1}\right) / \Delta_{\omega_{i}, w^{-1} \omega_{i}}(x) ;
$$

since we will not need this formula, the proof is left to the reader.

Continuing with the proof of Theorem 2.19 , let us define, for $k=1, \ldots, \ell$ :

$$
x^{(k)}=x_{i_{k}}\left(t_{k}\right) \cdots x_{i_{\ell}}\left(t_{\ell}\right) \in N^{w_{k}^{-1}}, y^{(k)}=\pi_{-}\left(x^{(k)}\right), z^{(k)}=\overline{\overline{w_{k}^{-1}}} y^{(k)} .
$$

Applying (2.47) with $x$ replaced by $x^{(k)}$ yields

$$
t_{k}=\left[z^{(k+1)}\right]_{0}^{\omega_{i_{k}}-\alpha_{i_{k}}}\left[z^{(k)}\right]_{0}^{-\omega_{i_{k}}} .
$$

On the other hand, combining the definition (1.10) with (2.27), we can rewrite (2.45) as follows:

$$
t_{k}=\left[\overline{\overline{w_{k+1}^{-1}}} y\right]_{0}^{\omega_{i_{k}}-\alpha_{i_{k}}}\left[\overline{\overline{w_{k}^{-1}}} y\right]_{0}^{-\omega_{i_{k}}} .
$$

Comparing (2.53) with (2.52), we see that Theorem 2.19 would follow from the equality $\left[\overline{\overline{w_{k}^{-1}}} y\right]_{0}=\left[z^{(k)}\right]_{0}$. The latter is obtained by observing that $\overline{\overline{w_{k}^{-1}}} y=$ $\overline{\overline{w_{k}^{-1}}} \overline{\bar{w}}^{-1} z=\tilde{y} z^{(k)}$, where $\tilde{y}=y_{\left(i_{1}, \ldots, i_{k-1}\right)}\left(p_{1}, \ldots, p_{k-1}\right) \in N_{-}$(this $\tilde{y}$ was denoted by $y^{\prime}$ in (2.36)).

2.7. Totally positive bases for $N_{-}(w)$. Although most of the results in this section were obtained in [4], we prefer to give independent proofs here; in some cases, this will allow us to refine the statements in [4].

We start with the following general definition.

Definition 2.21. Let $F$ be a finite collection of functions on a set $X$. A subset $\mathcal{B} \subset F$ is called a totally positive base for $F$ if $\mathcal{B}$ is a minimal (with respect to inclusion) subset of $F$ with the property that every $f \in F$ is a subtraction-free expression (i.e., a ratio of two polynomials with nonnegative integer coefficients) in the elements of $\mathcal{B}$.

For every $w \in W$, let us denote

$$
F(w)=\left\{\Delta_{w^{\prime \prime} \omega_{i}, w^{\prime} \omega_{i}}(y): i \in[1, r], w^{\prime} \preceq w^{\prime \prime} \preceq w^{-1}\right\} .
$$

(As earlier in (1.23), $w^{\prime} \preceq w^{\prime \prime}$ stands for $\ell\left(w^{\prime \prime}\right)=\ell\left(w^{\prime}\right)+\ell\left(w^{\prime-1} w^{\prime \prime}\right)$.) To every reduced word $\mathbf{i}=\left(i_{1}, \ldots, i_{m}\right) \in R(w)$ we associate three collections of regular functions on the group $N_{-}(w)$ :

$$
\begin{aligned}
F_{1}(\mathbf{i}) & =\left\{\Delta_{w_{k} \omega_{i_{k}}, \omega_{i_{k}}}: 1 \leq k \leq m\right\}, \\
F_{2}(\mathbf{i}) & =\left\{\Delta_{w^{-1} \omega_{i_{k}}, w_{k} \omega_{i_{k}}}: 2 \leq k \leq m+1\right\}, \\
F(\mathbf{i}) & =\left\{\Delta_{w_{k} \omega_{i}, w_{l} \omega_{i}}: i \in[1, r], 1 \leq k \leq l \leq m+1\right\},
\end{aligned}
$$

where $w_{k}=s_{i_{m}} \cdots s_{i_{k}}$ (cf. (2.34)). 
Theorem 2.22. For any reduced word $\mathbf{i}=\left(i_{1}, \ldots, i_{m}\right) \in R(w)$, each of the collections $F_{1}(\mathbf{i})$ and $F_{2}(\mathbf{i})$ of regular functions on $N_{-}(w)$ is a transcendence basis for $\mathbb{C}\left(N_{-}(w)\right)$ and a totally positive base for $F(w)$.

Proof. Let us first deal with $F_{1}(\mathbf{i})$. The most important part of the proof is to show that every minor in $F(w)$ is a subtraction-free expression in the minors from $F_{1}(\mathbf{i})$. Since we obviously have

$$
F(w)=\bigcup_{\mathbf{i} \in R(w)} F(\mathbf{i})
$$

this statement will directly follow from Lemmas 2.23 and 2.24 below.

Lemma 2.23. For any two reduced words $\mathbf{i}, \mathbf{i}^{\prime} \in R(w)$, every minor in $F_{1}\left(\mathbf{i}^{\prime}\right)$ is a subtraction-free expression in the minors from $F_{1}(\mathbf{i})$.

Proof. This is an immediate corollary of [4, Corollary 6.7]. The proof in [4] is based on repeated applications of determinantal identities of Theorem 1.16.

Lemma 2.24. Every minor in $F(\mathbf{i})$ is a subtraction-free expression in the minors from $F_{1}(\mathbf{i})$.

Proof. We need to show that every minor $\Delta_{w_{k} \omega_{i}, w_{l} \omega_{i}}$, for $i \in[1, r]$ and $1 \leq k \leq$ $l \leq m+1$, is a subtraction-free expression in the minors $\Delta_{w_{k} \omega_{i}, \omega_{i}}$. Recall that, by convention, $w_{m+1}=e$, so the statement is trivial for $l=m+1$. By (2.37), it also holds for $k=l$, since the corresponding minor equals 1 . Thus we may assume that $1 \leq k<l \leq m$; increasing $k$ and $l$ if necessary, we can also assume without loss of generality that $i_{k}=i_{l}=i$.

Let us arrange all the pairs $(k, l)$ with $1 \leq k \leq l \leq m+1$ in the following order: $\left(k^{\prime}, l^{\prime}\right)<(k, l)$ if either $l^{\prime}>l$, or $l^{\prime}=l, k^{\prime}>k$. Using induction with respect to this linear order, it is enough to show that for every $(k, l)$ such that $1 \leq k<l \leq m$ and $i_{k}=i_{l}=i$, the minor $\Delta_{w_{k} \omega_{i}, w_{l} \omega_{i}}$ is a subtraction-free expression in the minors $\Delta_{w_{k^{\prime}} \omega_{j}, w_{l^{\prime}} \omega_{j}}$ with $j \in[1, r]$ and $\left(k^{\prime}, l^{\prime}\right)<(k, l)$. The latter follows from the identity (1.25) applied to $u=w_{k+1}$ and $v=w_{l+1}$. Indeed, this identity can be rewritten as

$$
\Delta_{w_{k} \omega_{i}, w_{l} \omega_{i}}=\frac{\Delta_{w_{k} \omega_{i}, w_{l+1} \omega_{i}} \Delta_{w_{k+1} \omega_{i}, w_{l} \omega_{i}}+\prod_{j \neq i} \Delta_{w_{k+1} \omega_{j}, w_{l+1} \omega_{j}}^{-a_{j i}}}{\Delta_{w_{k+1} \omega_{i}, w_{l+1} \omega_{i}}},
$$

providing a desired subtraction-free expression.

Lemma 2.24 implies in particular that each minor $\Delta_{w_{k} \omega_{i_{k}}, w_{k+1} \omega_{i_{k}}}(y)$ is a rational function of the minors from $F_{1}(\mathbf{i})$. By Proposition 2.11, it follows that $F_{1}(\mathbf{i})$ is a transcendence basis for $\mathbb{C}\left(N_{-}(w)\right)$, hence it is a totally positive base for $F(w)$.

To prove that $F_{2}(\mathbf{i})$ has the same properties, we will apply the anti-automorphism $\tau_{w}$ of $G$ given by

$$
\tau_{w}(y)=\bar{w}\left(y^{-1}\right)^{\theta} \bar{w}^{-1},
$$

where $\theta$ was defined in (1.11). In view of (2.4), if $\tau_{w}(y)=y^{\prime}$, then

$$
y=\tau_{w^{-1}}\left(y^{\prime}\right), \quad \tau_{w}\left(y^{T}\right)=y^{\prime T} .
$$

A straightforward check shows that

$$
\tau_{w^{-1}}\left(N_{+}(w)\right)=N_{+}\left(w^{-1}\right), \quad \tau_{w}\left(N_{-}(w)\right)=N_{-}\left(w^{-1}\right) .
$$


Lemma 2.25. Let $y \in G$, and let $y^{\prime}=\tau_{w}(y)$ for some $w \in W$. For any $w^{\prime}, w^{\prime \prime} \in W$ such that $w^{\prime} \preceq w^{-1}$ and $w^{\prime \prime} \preceq w^{-1}$, we have

$$
\Delta_{w^{\prime \prime} \omega_{i}, w^{\prime} \omega_{i}}(y)=\Delta_{w w^{\prime} \omega_{i}, w w^{\prime \prime} \omega_{i}}\left(y^{\prime}\right) .
$$

Proof. In view of (1.9) and (2.4), the conditions $w^{\prime} \preceq w^{-1}$ and $w^{\prime \prime} \preceq w^{-1}$ imply that

$$
\overline{\overline{\left(w w^{\prime}\right)^{-1}}}={\overline{\overline{w^{\prime}}}}^{-1} \bar{w}^{-1}, \overline{w w^{\prime \prime}}=\bar{w} \overline{\overline{w^{\prime \prime}}} .
$$

Combining this with (2.15), we obtain:

$$
\begin{aligned}
\Delta_{w w^{\prime} \omega_{i}, w w^{\prime \prime} \omega_{i}}\left(y^{\prime}\right) & =\Delta^{\omega_{i}}\left(\overline{\overline{\left(w w^{\prime}\right)^{-1}}} y^{\prime} \overline{w w^{\prime \prime}}\right)=\Delta^{\omega_{i}}\left({\overline{\overline{w^{\prime}}}}^{-1} \bar{w}^{-1} y^{\prime} \overline{\bar{w}} \overline{\overline{w^{\prime \prime}}}\right) \\
& =\Delta^{\omega_{i}}\left(\overline{\overline{w^{\prime}}}-1\left(y^{-1}\right)^{\theta} \overline{\overline{w^{\prime \prime}}}\right)=\Delta^{\omega_{i}}\left(\overline{\overline{w^{\prime \prime}-1}} y \overline{w^{\prime}}\right)=\Delta_{w^{\prime \prime} \omega_{i}, w^{\prime} \omega_{i}}(y),
\end{aligned}
$$

as claimed.

By Lemma 2.25, the anti-automorphism $\tau_{w}$ transforms $F(w)$ into $F\left(w^{-1}\right)$, and $F_{2}(\mathbf{i})$ into $F_{1}\left(\mathbf{i}^{*}\right)$, where $\mathbf{i}^{*}=\left(i_{m}, \ldots, i_{1}\right) \in R\left(w^{-1}\right)$ is $\mathbf{i}$ written backwards. Thus the fact that $F_{2}(\mathbf{i})$ is a transcendence basis for $\mathbb{C}\left(N_{-}(w)\right)$ and a totally positive base for $F(w)$, follows from the same properties for $F_{1}(\mathbf{i})$ that we already proved. This completes the proof of Theorem 2.22.

2.8. Total positivity in $y$-coordinates. Let $N_{\geq 0} \subset N$ denote the multiplicative semigroup generated by the elements $x_{i}(t)$ for $i \in[1, r]$ and $t>0$. For every $w \in W$, let us denote (cf. [4])

$$
N_{>0}^{w}=N_{\geq 0} \cap N^{w}=N_{\geq 0} \cap B_{-} w B_{-} .
$$

The following analogue of Theorem 1.3 is due to G. Lusztig [18] (cf. Proposition 2.18).

Proposition 2.26. For any $w \in W$ and any reduced word $\mathbf{i}=\left(i_{1}, \ldots, i_{\ell}\right) \in R(w)$, the map $\left(t_{1}, \ldots, t_{\ell}\right) \mapsto x_{i_{1}}\left(t_{1}\right) \cdots x_{i_{\ell}}\left(t_{\ell}\right)$ restricts to a bijection $\mathbb{R}_{>0}^{\ell} \rightarrow N_{>0}^{w}$.

We will use Theorem 2.22 to obtain the following criteria for total positivity.

Theorem 2.27. Let $x \in N^{w}$, let $y=\pi_{-}(x) \in N_{-}(w)$, and let $\mathbf{i}=\left(i_{1}, \ldots, i_{\ell}\right) \in$ $R(w)$. Then the following conditions are equivalent:

(1) $x \in N_{>0}^{w}$;

(2) $\Delta(y)>0$ for any $\Delta \in F(w)$;

(3) $\Delta(y)>0$ for any $\Delta \in F_{1}(\mathbf{i})$;

(4) $\Delta(y)>0$ for any $\Delta \in F_{2}(\mathbf{i})$.

Proof. The equivalence of (2), (3) and (4) is immediate from Theorem 2.22. Let us show the equivalence of (1) and (3). By Proposition 2.26, every $x \in N_{>0}^{w}$ is of the form $x=x_{i_{1}}\left(t_{1}\right) \cdots x_{i_{\ell}}\left(t_{\ell}\right)$ for some $t_{1}, \ldots, t_{\ell}>0$. By Theorem 2.19, each $t_{k}$ is a monomial in $\ell$ variables $\left\{\Delta(y): \Delta \in F_{1}(\mathbf{i})\right\}$. It follows that the monomial transformation from $\left\{\Delta(y): \Delta \in F_{1}(\mathbf{i})\right\}$ to $\left\{t_{1}, \ldots, t_{\ell}\right\}$ is invertible (an explicit expression for the inverse transformation was given in [4, Theorem 4.3] but we will not need it here). Thus every $\Delta(y)$ with $\Delta \in F_{1}(\mathbf{i})$ is a Laurent monomial in $t_{1}, \ldots, t_{\ell}$. Hence $\Delta(y)>0$, and $(1) \Rightarrow(3)$ is proved.

To prove $(3) \Rightarrow(1)$, suppose that $\Delta(y)>0$ for $\Delta \in F_{1}(\mathbf{i})$. Let us define $t_{1}, \ldots, t_{\ell}$ via $(2.45)$, and let $\tilde{x}=x_{i_{1}}\left(t_{1}\right) \cdots x_{i_{\ell}}\left(t_{\ell}\right) \in N_{>0}^{w}$. Setting $\tilde{y}=\pi_{-}(\tilde{x})$, we see that 
$\Delta(y)=\Delta(\tilde{y})$ for any $\Delta \in F_{1}(\mathbf{i})$. By Lemma 2.24, we have $\Delta(y)=\Delta(\tilde{y})$ for any $\Delta \in F(\mathbf{i})$. In particular, $\Delta_{w_{k} \omega_{i_{k}}, w_{k+1} \omega_{i_{k}}}(y)=\Delta_{w_{k} \omega_{i_{k}}, w_{k+1} \omega_{i_{k}}}(\tilde{y})$ for $k=1, \ldots, \ell$. Using (2.35), we conclude that $y=\tilde{y}$ and so $x=\tilde{x} \in N_{>0}^{w}$, proving (3) $\Rightarrow$ (1).

Corollary 2.28. The map $\tau_{w}: N_{-}(w) \rightarrow N_{-}\left(w^{-1}\right)$ (cf. (2.58)) restricts to a bijection $\pi_{-}\left(N_{>0}^{w}\right) \rightarrow \pi_{-}\left(N_{>0}^{w^{-1}}\right)$.

Proof. We have already observed that by Lemma $2.25, \tau_{w}$ transforms $F(w)$ into $F\left(w^{-1}\right)$. The corollary then follows from Theorem 2.27.

We will now show that using $y$-coordinates (i.e., passing from a double Bruhat cell $G^{u, v}$ to the open subset $G_{0}^{u, v}$ ) will not create problems in the study of totally positive varieties. The following proposition is due to G. Lusztig [18]; for the convenience of the reader, we provide a proof.

Proposition 2.29. We have $N_{\geq 0}=N \cap G_{\geq 0}, N_{\geq 0}^{T}=N_{-} \cap G_{\geq 0}$ and $G_{\geq 0}=$ $N_{\geq 0}^{T} H_{>0} N_{\geq 0}$. In particular, $G_{\geq 0} \subset G_{0}$, i.e., any totally nonnegative element in $G$ admits the Gaussian decomposition. Furthermore, for any $u, v \in W$, the totally positive variety $G_{>0}^{u, v}$ decomposes as

$$
G_{>0}^{u, v}=\left(N_{>0}^{u^{-1}}\right)^{T} H_{>0} N_{>0}^{v},
$$

in the notation of (2.61).

Proof. By the definition of $G_{\geq 0}$, every totally nonnegative element $x \in G$ has the form (cf. (1.3)) $x=x_{\mathbf{i}}\left(a ; t_{1}, \ldots, t_{m}\right)$, where $\mathbf{i}=\left(i_{1}, \ldots, i_{m}\right)$ is some word in the alphabet $[1, r] \cup[\overline{1}, \bar{r}]$, the $t_{k}$ are positive real numbers, and $a \in H_{>0}$. We say that $\mathbf{i}$ is unmixed if all the indices from $[\overline{1}, \bar{r}]$ precede those from $[1, r]$. By repeated application of the commutation relations (2.5), (2.9) and (2.11), we can transform $x$ to the form $x=x_{\mathbf{i}^{\prime}}\left(a^{\prime} ; t_{1}^{\prime}, \ldots, t_{m}^{\prime}\right)$ for an unmixed word $\mathbf{i}^{\prime}, a^{\prime} \in H_{>0}$, and all $t_{k}^{\prime}>0$. This proves the decomposition $G_{\geq 0}=N_{\geq 0}^{T} H_{>0} N_{\geq 0}$. The equalities $N_{\geq 0}=N \cap G_{\geq 0}$ and $N_{\geq 0}^{T}=N_{-} \cap G_{\geq 0}$ follow from this decomposition of $G_{\geq 0}$ and the uniqueness of the Gaussian decomposition. Finally, (2.62) is proved by the same argument combined with (2.44).

Combining Propositions 2.29 and 2.15 with Theorem 2.27, we obtain the following description of the totally positive variety $G_{>0}^{u, v}$ in terms of $y$-coordinates.

Theorem 2.30. An element $x \in G_{0}^{u, v}$ lies in $G_{>0}^{u, v}$ if and only if its $y$-coordinates $\left(y_{+}, y_{0}, y_{-}\right)$satisfy the following properties:

- $\Delta\left(y_{-}\right)>0$ for any $\Delta \in F(v)$;

- $\Delta\left(y_{+}^{T}\right)>0$ for any $\Delta \in F\left(u^{-1}\right)$;

- $y_{0} \in H_{>0}$.

\section{Proofs of the MAin Results}

This section contains proofs of the main results in Section 1.

\subsection{Proofs of Theorems 1.1, 1.2, and 1.3.}

Proof of Theorem 1.1. We will explicitly construct a desired biregular isomorphism of $G^{u, v}$ with a Zariski open subset of $\mathbb{C}^{r+\ell(u)+\ell(v)}$ with the help of a "twisted" 
version of $y$-coordinates (cf. Section 2.5). We fix a representative $\tilde{u}$ of $u$, and associate to any $x \in G^{u, v}$ a triple $\left(y_{(+)}, y_{(0)}, y_{(-)}\right)$given by

$$
y_{(+)}=\pi_{+}(x), y_{(-)}=\pi_{-}\left(x^{-1}\right), y_{(0)}=\left[\tilde{u}^{-1} x\right]_{0} ;
$$

in view of Propositions 2.9 and 2.10, this triple is well defined and belongs to $N_{+}(u) \times H \times N_{-}\left(v^{-1}\right)$. Our statement is a consequence of the following.

Proposition 3.1. The correspondence $x \mapsto\left(y_{(+)}, y_{(0)}, y_{(-)}\right)$given by (3.1) is a biregular isomorphism of $G^{u, v}$ with the Zariski open subset of $N_{+}(u) \times H \times N_{-}\left(v^{-1}\right)$ consisting of triples such that $y_{(-)} y_{(+)} \in v G_{0} u^{-1}$.

Proof. The proof is essentially the same as that of Proposition 2.14; the inverse of the correspondence (3.1) is given by

$$
x=y_{(+)} \tilde{u}\left[\tilde{v}^{-1} y_{(-)} y_{(+)} \tilde{u}\right]_{+}^{-1} y_{(0)},
$$

where $\tilde{v}$ is any representative of $v$.

Proof of Theorem 1.2. By Theorem 1.1, every double cell $G^{u, v}$ is smooth, and $\operatorname{dim}\left(G^{u, v}\right)=\operatorname{dim}\left(H \times \mathbb{C}^{m}\right)$. It follows from the Grothendieck-Zariski factorization theorem [14, Theorem 8.12.6] that if $X$ and $Y$ are irreducible algebraic varieties over $\mathbb{C}$ of the same dimension, and $Y$ is smooth, then any injective morphism from $X$ to $Y$ is an open embedding. Therefore, Theorem 1.2 is implied by the following proposition. (We thank Michel Brion for explaining this implication to us and providing the reference [14].)

Proposition 3.2. For every $u, v \in W$ and $\mathbf{i}=\left(i_{1}, \ldots, i_{m}\right) \in R(u, v)$, the map $x_{\mathbf{i}}$ restricts to an injective regular map $H \times \mathbb{C}_{\neq 0}^{m} \rightarrow G^{u, v}$.

Proof. First let us show that $x_{\mathbf{i}}\left(H \times \mathbb{C}_{\neq 0}^{m}\right) \subset G^{u, v}$. We will show that $x_{\mathbf{i}}\left(H \times \mathbb{C}_{\neq 0}^{m}\right) \subset$ $B_{-} v B_{-}$; the inclusion $x_{\mathbf{i}}\left(H \times \mathbb{C}_{\neq 0}^{m}\right) \subset B u B$ is proved similarly (or deduced from the previous one with the help of the transpose map). Let

$$
\left\{k_{1}<\cdots<k_{\ell}\right\}=\left\{k \in[1, m]: i_{k} \in[1, r]\right\},
$$

so that $\left(i_{k_{1}}, \ldots, i_{k_{\ell}}\right) \in R(v)$. Let us use the fact that, for every $i \in[1, r]$ and nonzero $t \in \mathbb{C}$, we have $x_{i}(t) \in B_{-} s_{i} B_{-}$and $x_{\bar{i}}(t) \in B_{-}$(cf. (2.12)). It follows that if $\left(a ; t_{1}, \ldots, t_{m}\right) \in H \times \mathbb{C}_{\neq 0}^{m}$, then

$$
x_{\mathbf{i}}\left(a ; t_{1}, \ldots, t_{m}\right) \in B_{-} \cdot B_{-} s_{i_{k_{1}}} B_{-} \cdot B_{-} \cdots B_{-} \cdot B_{-} s_{i_{k_{\ell}}} B_{-} \cdot B_{-}=B_{-} v B_{-},
$$

as desired; the last equality follows from the well-known fact that

$$
B_{-} w^{\prime} B_{-} \cdot B_{-} w^{\prime \prime} B_{-}=B_{-} w^{\prime} w^{\prime \prime} B_{-}
$$

whenever $\ell\left(w^{\prime} w^{\prime \prime}\right)=\ell\left(w^{\prime}\right)+\ell\left(w^{\prime \prime}\right)$ (cf. [5, IV.2.4]).

It remains to show that the map $x_{\mathbf{i}}: H \times \mathbb{C}_{\neq 0}^{m} \rightarrow G^{u, v}$ is injective. There is nothing to prove if $u=v=e$, so we can assume that $m=\ell(u)+\ell(v) \geq 1$. Suppose that $i_{m}=i \in[1, r]$ (the case when $i_{m} \in[\overline{1}, \bar{r}]$ is treated in the same way). Denote $v^{\prime}=v s_{i}$ so that $\mathbf{i}^{\prime}=\left(i_{1}, \ldots, i_{m-1}\right) \in R\left(u, v^{\prime}\right)$. Now suppose

$$
x_{\mathbf{i}}\left(a ; t_{1}, \ldots, t_{m}\right)=x_{\mathbf{i}}\left(a^{\prime} ; t_{1}^{\prime}, \ldots, t_{m}^{\prime}\right),
$$

where $\left(a ; t_{1}, \ldots, t_{m}\right)$ and $\left(a^{\prime} ; t_{1}^{\prime}, \ldots, t_{m}^{\prime}\right)$ belong to $H \times \mathbb{C}_{\neq 0}^{m}$. Multiplying both sides of the last equality on the right by $x_{i}\left(-t_{m}^{\prime}\right)$, we obtain:

$$
x_{\mathbf{i}}\left(a ; t_{1}, \ldots, t_{m-1}, t_{m}-t_{m}^{\prime}\right)=x_{\mathbf{i}^{\prime}}\left(a^{\prime} ; t_{1}^{\prime}, \ldots, t_{m-1}^{\prime}\right) .
$$


If $t_{m} \neq t_{m}^{\prime}$, then the left-hand side of (3.2) lies in $G^{u, v}$, while the right-hand side lies in $G^{u, v^{\prime}}$. But this is impossible because the double Bruhat cells are disjoint. Thus $t_{m}=t_{m}^{\prime}$, and the desired injectivity follows by induction on $m$. This completes the proof of Proposition 3.2 and Theorem 1.2.

Proof of Theorem 1.3. If a double reduced word $\mathbf{i} \in R(u, v)$ is unmixed, i.e., all the indices from $[\overline{1}, \bar{r}]$ precede those from $[1, r]$, then our statement follows by combining (2.62) with Proposition 2.26. The statement for an arbitrary $\mathbf{i} \in R(u, v)$ can be reduced to the case of an unmixed $\mathbf{i}$ by the argument used in the proof of Proposition 2.29, i.e., by repeated application of the commutation relations (2.5), (2.9) and (2.11).

\subsection{Proofs of Theorems $\mathbf{1 . 6}$ and $\mathbf{1 . 7 .}$}

Proof of Theorem 1.6. The fact that the right-hand side of (1.12) is well defined for any $x \in G^{u, v}$ follows from Propositions 2.9 and 2.10. Let us show that $x^{\prime}=$ $\zeta^{u, v}(x) \in G^{u^{-1}, v^{-1}}$. Using (2.31), we can rewrite $x^{\prime}$ as

$$
x^{\prime}=\overline{\bar{u}}^{-1}\left(y_{+}^{-1}\left[x \overline{v^{-1}}\right]_{-}\left[x \overline{v^{-1}}\right]_{0}\right)^{\theta},
$$

where $y_{+}=\pi_{+}(x)$. It follows that $\overline{\bar{u}} x^{\prime} \in G_{0}$, and

$$
\left[\overline{\bar{u}} x^{\prime}\right]_{-}=\left(y_{+}^{-1}\right)^{\theta} .
$$

Hence $\left[\overline{\bar{u}} x^{\prime}\right]_{-} \in N_{+}(u)^{\theta}=N_{-}\left(u^{-1}\right)$, and we conclude from Proposition 2.9 that $x^{\prime} \in B u^{-1} B$. The inclusion $x^{\prime} \in B_{-} v^{-1} B_{-}$is proved in a similar way (or by using the transpose map); the counterpart of (3.3) is given by

$$
\left[x^{\prime} \bar{v}\right]_{+}=\left(y_{-}^{-1}\right)^{\theta},
$$

where $y_{-}=\pi_{-}(x)(\mathrm{cf} .(2.32))$.

We have proved that $x^{\prime} \in G^{u^{-1}, v^{-1}}$. To complete the proof of Theorem 1.6, it suffices to show that $\zeta^{u^{-1}, v^{-1}}\left(\zeta^{u, v}(x)\right)=x$ for any $x \in G^{u, v}$. Notice that (3.3) and (3.4) can be rewritten as

$$
\left[\overline{\bar{u}} x^{\prime}\right]_{-}=\overline{\bar{u}}\left(\left[\overline{\overline{u^{-1}}} x\right]_{-}^{-1}\right)^{\theta} \overline{\bar{u}}^{-1},\left[x^{\prime} \bar{v}\right]_{+}=\bar{v}^{-1}\left(\left[x \overline{v^{-1}}\right]_{+}^{-1}\right)^{\theta} \bar{v} .
$$

The desired equality $\zeta^{u^{-1}, v^{-1}}\left(x^{\prime}\right)=x$ follows by substituting these expressions into the expression for $\zeta^{u^{-1}, v^{-1}}\left(x^{\prime}\right)$ obtained from (1.12).

The following proposition shows that the twist map respects the Gaussian decomposition.

Proposition 3.3. The twist map $\zeta^{u, v}: x \mapsto x^{\prime}$ sends the open subset $G_{0}^{u, v}$ to $G_{0}^{u^{-1}, v^{-1}}$, and we have

$$
\left[x^{\prime}\right]_{0}=\left[\overline{\overline{u^{-1}}} x\right]_{0}^{-1}[x]_{0}\left[x \overline{v^{-1}}\right]_{0}^{-1} .
$$

Proof. To show that $x$ and $x^{\prime}$ belong to $G_{0}$ simultaneously, it suffices to rewrite (1.12) as

$$
x^{\prime}=\left(\left[\overline{\overline{u^{-1}}} x\right]_{0}\left[\overline{\overline{u^{-1}}} x\right]_{+} x^{-1}\left[x \overline{v^{-1}}\right]_{-}\left[x \overline{v^{-1}}\right]_{0}\right)^{\theta} .
$$

In view of (2.3), this also implies (3.6). 
Let us now describe the twist map in terms of $y$-coordinates. Recall the definition (2.56) of the anti-automorphism $\tau_{w}$ of the group $G$.

Proposition 3.4. Suppose $x \in G_{0}^{u, v}$ has y-coordinates $\left(y_{+}, y_{0}, y_{-}\right)$. Then the $y$ coordinates $\left(y_{+}^{\prime}, y_{0}^{\prime}, y_{-}^{\prime}\right)$ of $x^{\prime}=\zeta^{u, v}(x)$ are given by

$$
\begin{aligned}
& y_{+}^{\prime}=\tau_{u^{-1}}\left(y_{+}\right), \\
& y_{0}^{\prime}=\left[y_{+} \bar{u}\right]_{0} y_{0}^{-1}\left[\overline{\bar{v}} y_{-}\right]_{0}, \\
& y_{-}^{\prime}=\tau_{v}\left(y_{-}\right) .
\end{aligned}
$$

Proof. The desired expressions for $y_{+}^{\prime}$ and $y_{-}^{\prime}$ follow from (3.5); the expression for $y_{0}^{\prime}$ follows from (3.6) combined with (2.42).

Proof of Theorem 1.7. Let $x \in G_{>0}^{u, v}$, and let $x^{\prime}=\zeta^{u, v}(x)$. By Proposition 2.29, $x \in G_{0}^{u, v}$ so $x$ has well-defined $y$-coordinates $\left(y_{+}, y_{0}, y_{-}\right)$. By Proposition 3.4, the $y$-coordinates $\left(y_{+}^{\prime}, y_{0}^{\prime}, y_{-}^{\prime}\right)$ of $x^{\prime}$ are given by (3.7). By Theorem 2.30, the triple $\left(y_{+}, y_{0}, y_{-}\right)$satisfies the properties given there, and it suffices to check that $\left(y_{+}^{\prime}, y_{0}^{\prime}, y_{-}^{\prime}\right)$ satisfies the same properties with $(u, v)$ replaced by $\left(u^{-1}, v^{-1}\right)$. In view of (3.7) and (2.59), if $\Delta\left(y_{-}\right)>0$ for any $\Delta \in F(v)$, then $\Delta\left(y_{-}^{\prime}\right)>0$ for any $\Delta \in F\left(v^{-1}\right)$. Similarly, using (2.57) we obtain that if $\Delta\left(y_{+}^{T}\right)>0$ for any $\Delta \in F\left(u^{-1}\right)$, then $\Delta\left(y_{+}^{\prime}\right)>0$ for any $\Delta \in F(u)$.

It remains to show that $y_{0}^{\prime} \in H_{>0}$. Applying the character $a \rightarrow a^{\omega_{i}}$ to both sides of the equality $y_{0}^{\prime}=\left[y_{+} \bar{u}\right]_{0} y_{0}^{-1}\left[\overline{\bar{v}} y_{-}\right]_{0}$ in $(3.7)$, we obtain

$$
\left(y_{0}^{\prime}\right)^{\omega_{i}}=\Delta_{u \omega_{i}, \omega_{i}}\left(y_{+}^{T}\right) y_{0}^{-\omega_{i}} \Delta_{v^{-1} \omega_{i}, \omega_{i}}\left(y_{-}\right) .
$$

Since $\Delta_{v^{-1} \omega_{i}, \omega_{i}} \in F(v)$ and $\Delta_{u \omega_{i}, \omega_{i}} \in F\left(u^{-1}\right)$, it follows that $\left(y_{0}^{\prime}\right)^{\omega_{i}}>0$ for any $i \in[1, r]$. Therefore, $y_{0}^{\prime} \in H_{>0}$, as desired.

3.3. Proof of Theorem 1.9. First notice that the equivalence of (1.19) and (1.20) follows by applying the character $a \mapsto a^{\omega_{i}}$ to both sides of (1.20) and simplifying the result. In proving (1.18) and (1.20), we will follow the same strategy that was used in the proof of Theorem 1.3: first treat the case when $\mathbf{i}$ is unmixed, and then extend the result to the general case with the help of commutation relations (2.5), (2.9) and (2.11).

Let us first assume that $\mathbf{i} \in R(u, v)$ is unmixed, i.e., all the indices from $[\overline{1}, \bar{r}]$ precede those from $[1, r]$. Repeatedly using (2.5), we conclude that in this case $x=x_{\mathbf{i}}\left(a ; t_{1}, \ldots, t_{m}\right) \in G_{0}^{u, v}$, and the components in the Gaussian decomposition of $x$ are given by

$$
\begin{aligned}
& {[x]_{-}=x_{i_{1}}\left(a^{-\alpha_{\left|i_{1}\right|}} t_{1}\right) \cdots x_{i_{\ell(u)}}\left(a^{-\alpha_{\mid i} \ell(u) \mid} t_{\ell(u)}\right),} \\
& {[x]_{0}=a,[x]_{+}=x_{i_{\ell(u)+1}}\left(t_{\ell(u)+1}\right) \cdots x_{i_{m}}\left(t_{m}\right) .}
\end{aligned}
$$

Since by Theorem 1.6, $x=\zeta^{u^{-1}, v^{-1}}\left(x^{\prime}\right)$, formula (3.6) implies that

$$
a=[x]_{0}=\left[\overline{\bar{u}} x^{\prime}\right]_{0}^{-1}\left[x^{\prime}\right]_{0}\left[x^{\prime} \bar{v}\right]_{0}^{-1} .
$$

This proves (1.20) since a simple inspection shows that the right-hand side of (3.9) is equal to that of (1.20) when $\mathbf{i}$ is unmixed.

Turning to the proof of (1.18), let us first consider the case $\ell(u)<k \leq m$. Let $\left(y_{+}, y_{0}, y_{-}\right)$be the $y$-coordinates of $x$, and $\left(y_{+}^{\prime}, y_{0}^{\prime}, y_{-}^{\prime}\right)$ be the $y$-coordinates of $x^{\prime}$. 
By (2.45), we have

$$
t_{k}=\frac{1}{\Delta_{v^{-1} v_{<k} \omega_{i_{k}}, \omega_{i_{k}}}\left(y_{-}\right) \Delta_{v^{-1} v_{<k+1} \omega_{i_{k}}, \omega_{i_{k}}}\left(y_{-}\right)} \prod_{j \neq i_{k}} \Delta_{v^{-1} v_{<k+1} \omega_{j}, \omega_{j}}\left(y_{-}\right)^{-a_{j, i_{k}}} .
$$

Using (3.7), (2.59), (2.22), (2.41), and (2.42), we can rewrite $\Delta_{v^{-1} v_{<k} \omega_{j}, \omega_{j}}\left(y_{-}\right)$as follows:

$$
\begin{aligned}
& \Delta_{v^{-1} v_{<k} \omega_{j}, \omega_{j}}\left(y_{-}\right)=\Delta_{v \omega_{j}, v_{<k} \omega_{j}}\left(y_{-}^{\prime}\right)=\Delta_{\omega_{j}, v_{<k} \omega_{j}}\left(\overline{\overline{v^{-1}}} y_{-}^{\prime}\right) \\
& \quad=\Delta_{\omega_{j}, v_{<k} \omega_{j}}\left(\left[\overline{\overline{v^{-1}}} y_{-}^{\prime}\right]_{+}\right)\left[\overline{\overline{v^{-1}}} y_{-}^{\prime}\right]_{0}^{\omega_{j}}=\Delta_{\omega_{j}, v_{<k} \omega_{j}}\left(x^{\prime}\right)\left[y^{\prime}\right]_{0}^{-\omega_{j}}\left[\overline{\overline{v^{-1}}} y_{-}^{\prime}\right]_{0}^{\omega_{j}} \\
& \quad=\Delta_{\omega_{j}, v_{<k} \omega_{j}}\left(x^{\prime}\right) / \Delta_{\omega_{j}, v \omega_{j}}\left(x^{\prime}\right) .
\end{aligned}
$$

Substituting the expressions given by (3.11) into (3.10), we express $t_{k}$ as a Laurent monomial in the minors $\Delta_{l}\left(x^{\prime}\right)$ given by (1.16). Using the notation from Section 1.6, this monomial can be written as follows:

$$
t_{k}=\frac{1}{\Delta_{k}\left(x^{\prime}\right) \Delta_{k^{+}}\left(x^{\prime}\right)} \prod_{l: l^{-}<k<l} \Delta_{l}\left(x^{\prime}\right)^{-a_{\left|i_{l}\right|, i_{k}}} \prod_{j \in[1, r]} \Delta_{m+j}\left(x^{\prime}\right)^{a_{j, i_{k}}},
$$

where the index $k^{+}$is defined by $\left(k^{+}\right)^{-}=k$. Formula (1.18) now follows by simple inspection which shows that, for $\mathbf{i}$ unmixed, the right-hand side of (1.18) is equal to the one of (3.12).

The proof of (1.18) for $1 \leq k \leq \ell(u)$ is practically the same as above. In this case, the counterpart of (3.12) is given by

$$
a^{-\alpha_{\left|i_{k}\right|} \mid} t_{k}=\frac{1}{\Delta_{k}\left(x^{\prime}\right) \Delta_{k^{+}}\left(x^{\prime}\right)} \prod_{l: l^{-}<k<l} \Delta_{l}\left(x^{\prime}\right)^{-a_{\left|i_{l}\right|,\left|i_{k}\right|}} \prod_{l: l^{-}=0} \Delta_{l}\left(x^{\prime}\right)^{a_{\left|i_{l}\right|,\left|i_{k}\right|}} .
$$

To deduce (1.18) from (3.13), first notice that in view of (1.19) and (2.27), we have

$$
a^{\alpha_{\left|i_{k}\right|}}=\prod_{1 \leq l \leq m+r} \Delta_{l}\left(x^{\prime}\right)^{\left(\varepsilon\left(i_{l}\right)-\varepsilon\left(i_{l}-\right)\right) a_{\left|i_{l}\right|,\left|i_{k}\right|} / 2} .
$$

Thus in order to check (1.18), it suffices to show that, for $\mathbf{i}$ unmixed and $1 \leq k \leq$ $\ell(u)$, the right-hand side of (3.13) is equal to

$$
\prod_{l=1}^{m+r} \Delta_{l}\left(x^{\prime}\right)^{\left(\varepsilon\left(i_{l^{-}}\right) / 2+\chi\left(k, l^{-}\right)-\varepsilon\left(i_{l}\right) / 2-\chi(k, l)\right)} a_{\left|i_{l}\right|,\left|i_{k}\right|} ;
$$

this is again checked by direct inspection.

Now let us prove (1.18) and (1.20) for an arbitrary double reduced word for $u$ and $v$. Every such word can be obtained from an unmixed one by a sequence of mixed moves of the form

$$
\cdots \bar{j} i \cdots \rightsquigarrow \cdots i \bar{j} \cdots .
$$

It therefore suffices to prove the following statement.

Lemma 3.5. Suppose a reduced word $\mathbf{i}^{\prime} \in R(u, v)$ is obtained from $\mathbf{i} \in R(u, v)$ by a mixed move. If (1.18) and (1.20) hold for $\mathbf{i}$, then they also hold for $\mathbf{i}^{\prime}$.

Proof. Suppose $\mathbf{i}^{\prime}$ is obtained from $\mathbf{i}$ by interchanging $i_{k}=\bar{j}$ and $i_{k+1}=i$. By (2.5), (2.9) and (2.11), the factorization parameters that appear in two factorizations

$$
x=x_{\mathbf{i}}\left(a ; t_{1}, \ldots, t_{m}\right)=x_{\mathbf{i}^{\prime}}\left(a^{\prime} ; t_{1}^{\prime}, \ldots, t_{m}^{\prime}\right)
$$


of an element $x \in G^{u, v}$ are related as follows. If $i \neq j$, then

$$
a^{\prime}=a, t_{l}^{\prime}=t_{l}(l \notin\{k, k+1\}), t_{k}^{\prime}=t_{k+1}, t_{k+1}^{\prime}=t_{k} .
$$

For $i=j$, a straightforward calculation using (2.5) and (2.11) shows that

$$
\begin{gathered}
\left(a^{\prime}\right)^{\omega_{p}}=a^{\omega_{p}}\left(1+t_{k} t_{k+1}\right)^{-\delta_{i, p}}, \\
t_{l}^{\prime}=t_{l}\left(1+t_{k} t_{k+1}\right)^{\varepsilon\left(i_{l}\right) a_{i,\left|i_{l}\right|} \mid}(l<k), \\
t_{k}^{\prime}=t_{k+1}\left(1+t_{k} t_{k+1}\right), t_{k+1}^{\prime}=t_{k}\left(1+t_{k} t_{k+1}\right)^{-1}, \\
t_{l}^{\prime}=t_{l}(l>k+1) .
\end{gathered}
$$

We need to show the following: if we substitute the parameters $t_{l}$ and $a^{\omega_{p}}$ given by (1.18) and (1.19) into (3.14)-(3.18), then the resulting $t_{l}^{\prime}$ and $\left(a^{\prime}\right)^{\omega_{p}}$ satisfy the same formulas (1.18) and (1.19) with $\mathbf{i}$ replaced by $\mathbf{i}^{\prime}$. This is immediate from the definitions when $i \neq j$, so let us assume $i=j$. By the definition (1.16), we have $\Delta_{l, \mathbf{i}}=\Delta_{l, \mathbf{i}^{\prime}}$ for $l \neq k+1$, so we will denote this minor simply by $\Delta_{l}$. The key calculation is now as follows.

Lemma 3.6. In the above notation, if $t_{k}$ and $t_{k+1}$ satisfy (1.18), then

$$
1+t_{k} t_{k+1}=\frac{\Delta_{k+1, \mathbf{i}}\left(x^{\prime}\right) \Delta_{k+1, \mathbf{i}}\left(x^{\prime}\right)}{\Delta_{k}\left(x^{\prime}\right) \Delta_{(k+1)^{+}}\left(x^{\prime}\right)} .
$$

Proof. Let us denote $u^{\prime}=u_{\geq k+2}$ and $v^{\prime}=v_{<k}$ (this is unambiguous since these expressions are the same for $\mathbf{i}$ and $\mathbf{i}^{\prime}$; cf. (1.15)). In view of (1.16), we have

$$
\begin{aligned}
& \Delta_{k+1, \mathbf{i}}=\Delta_{u^{\prime} \omega_{i}, v^{\prime} \omega_{i}}, \Delta_{k+1, \mathbf{i}^{\prime}}=\Delta_{u^{\prime} s_{i} \omega_{i}, v^{\prime} s_{i} \omega_{i}}, \\
& \Delta_{k}=\Delta_{u^{\prime} s_{i} \omega_{i}, v^{\prime} \omega_{i}}, \Delta_{(k+1)^{+}}=\Delta_{u^{\prime} \omega_{i}, v^{\prime} s_{i} \omega_{i}},
\end{aligned}
$$

so (3.19) takes the form

$$
1+t_{k} t_{k+1}=\frac{\Delta_{u^{\prime} \omega_{i}, v^{\prime} \omega_{i}}\left(x^{\prime}\right) \Delta_{u^{\prime} s_{i} \omega_{i}, v^{\prime} s_{i} \omega_{i}}\left(x^{\prime}\right)}{\Delta_{u^{\prime} s_{i} \omega_{i}, v^{\prime} \omega_{i}}\left(x^{\prime}\right) \Delta_{u^{\prime} \omega_{i}, v^{\prime} s_{i} \omega_{i}}\left(x^{\prime}\right)} .
$$

On the other hand, if $t_{k}$ and $t_{k+1}$ are given by (1.18), then

$$
t_{k} t_{k+1}=\prod_{l=1}^{m+r} \Delta_{l}\left(x^{\prime}\right)^{\left(\chi\left(k, l^{-}\right)+\chi\left(k+1, l^{-}\right)-\chi(k, l)-\chi(k+1, l)\right) a_{\left|i_{l}\right|, i} .}
$$

By (1.17), we have

$$
\chi(k, l)+\chi(k+1, l)= \begin{cases}1 & \text { if } l>k+1 \\ 1 / 2 & \text { if } l \in\{k, k+1\} \\ 0 & \text { if } l<k\end{cases}
$$

It follows that

$$
\begin{aligned}
t_{k} t_{k+1} & =\Delta_{k}\left(x^{\prime}\right)^{-1} \Delta_{(k+1)^{+}}\left(x^{\prime}\right)^{-1} \prod_{l: l^{-}<k<l} \Delta_{l}\left(x^{\prime}\right)^{-a_{\left|i_{l}\right|, i}} \\
& =\left(\Delta_{u^{\prime} s_{i} \omega_{i}, v^{\prime} \omega_{i}}\left(x^{\prime}\right) \Delta_{u^{\prime} \omega_{i}, v^{\prime} s_{i} \omega_{i}}\left(x^{\prime}\right)\right)^{-1} \prod_{p \neq i} \Delta_{u^{\prime} \omega_{p}, v^{\prime} \omega_{p}}\left(x^{\prime}\right)^{-a_{p i}} .
\end{aligned}
$$

Therefore, (3.21) becomes a consequence of (1.25), and we are done. 
If we substitute the expressions given by (1.18), (1.19) and (3.19) into the formulas (3.14)-(3.18), then an easy simplification shows that they will be given by (1.18) and (1.19) for $\mathbf{i}^{\prime}$. This completes the proofs of Lemma 3.5 and Theorem 1.9.

3.4. Proofs of Theorems $\mathbf{1 . 1 1}$ and $\mathbf{1 . 1 2}$. We start by recalling a well-known property of reduced words in Coxeter groups (cf. $[5,15])$. To state it, we will need the following notion.

We call a $d$-move the transformation of a reduced word that replaces $d$ consecutive entries $i, j, i, j, \ldots$ by $j, i, j, i, \ldots$, for some $i$ and $j$ such that $d$ is the order of $s_{i} s_{j}$. Note that, for given $i$ and $j$, the value of $d$ can be determined from the Cartan matrix as follows: if $a_{i j} a_{j i}=0$ (resp. 1,2,3), then $d=2($ resp. $3,4,6)$.

Proposition 3.7. Every two reduced words for the same element of a Coxeter group can be obtained from each other by a sequence of $d$-moves.

Applying this proposition to the group $W \times W$, we conclude that every two double reduced words $\mathbf{i}, \mathbf{i}^{\prime} \in R(u, v)$ can be obtained from each other by a sequence of the following operations: $d$-moves for each of the alphabets $[1, r]$ and $[\overline{1}, \bar{r}]$, and also mixed moves (cf. Section 3.3) and their inverses.

Proof of Theorem 1.12. Let us first prove that $F(\mathbf{i})$ is a transcendence basis for the field $\mathbb{C}\left(G^{u, v}\right)$. By Theorem 1.1, F(i) is of cardinality $\operatorname{dim} G^{u, v}$. It is therefore enough to show that $F(\mathbf{i})$ generates $\mathbb{C}\left(G^{u, v}\right)$. In view of Theorem 1.6, it suffices to show that the collection of "twisted" minors $\Delta_{k, \mathbf{i}}\left(x^{\prime}\right)$ (cf. (1.16)) generates $\mathbb{C}\left(G^{u, v}\right)$. By Theorem 1.2, the field $\mathbb{C}\left(G^{u, v}\right)$ is generated by the factorization parameters $t_{k}$ and $a^{\omega_{i}}$, and the claim follows by Theorem 1.9.

The second statement of the theorem is a consequence of the following lemma.

Lemma 3.8. Suppose a double reduced word $\mathbf{i}^{\prime}$ is obtained from $\mathbf{i}$ by a d-move in one of the alphabets $[1, r]$ and $[\overline{1}, \bar{r}]$, or by a mixed move, or by the inverse of a mixed move. Then each element of the set difference $F\left(\mathbf{i}^{\prime}\right) \backslash F(\mathbf{i})$ is a subtractionfree expression in the elements of $F(\mathbf{i})$.

Proof. For $d$-moves in $[1, r]$ or $[\overline{1}, \bar{r}]$, the desired subtraction-free expressions can be obtained from generalized Plücker relations in Theorem 1.16 (including the omitted relations of type $G_{2}$ ); this part of the argument is essentially borrowed from [4, Proposition 6.10]. For mixed moves and their inverses, the statement follows in the same way from Theorem 1.17 (cf. the proof of Lemma 3.5 above). Lemma 3.8 and Theorem 1.12 are proved.

Proof of Theorem 1.11. It will suffice to show that the following are equivalent:

(1) $x \in G_{>0}^{u, v}$

(2) $\Delta(x)>0$ for any $\Delta \in F(u, v)$;

(3) $\Delta(x)>0$ for any $\Delta \in F(\mathbf{i})$.

The equivalence of (2) and (3) follows from Theorem 1.12. Let us show that (1) $\Rightarrow(3)$. By Theorem 1.7, if $x \in G_{>0}^{u, v}$, then $x^{\prime} \in G_{>0}^{u^{-1}, v^{-1}}$. The condition (3) now follows by applying Theorems 1.3 and 1.10 to $x^{\prime}$ and the reduced word $\mathbf{i}^{*} \in R\left(u^{-1}, v^{-1}\right)$ opposite to $\mathbf{i}$.

It remains to show that $(2) \Rightarrow(1)$. First of all, by Corollary $2.5,(2)$ implies that $x \in G_{0}^{u, v}$; moreover, $[x]_{0} \in H_{>0}$. By Proposition 2.15 and Theorems 2.30 and 2.27, is suffices to show that $y_{-}=\pi_{-}(x)$ satisfies $\Delta_{v^{-1} \omega_{i}, v^{\prime} \omega_{i}}\left(y_{-}\right)>0$, for $i \in[1, r]$ and 
any $v^{\prime} \preceq v^{-1}$. Using (2.41) and (2.22), we obtain (cf. (3.11)):

$$
\Delta_{v^{-1} \omega_{i}, v^{\prime} \omega_{i}}\left(y_{-}\right)=\frac{\Delta_{\omega_{i}, v^{\prime} \omega_{i}}(x)}{\Delta_{\omega_{i}, v^{-1} \omega_{i}}(x)} .
$$

By (2), the right-hand side of (3.23) is positive, and the proof is complete.

\section{4. $G L_{n}$ THEORY}

Throughout this section, $G=G L_{n}(\mathbb{C})$ is the group of invertible $n \times n$ matrices with complex entries. In this case, the problems under consideration become quite natural questions in "classical" linear algebra, so we will formulate them hereand state our main results - in an elementary and self-contained way. We will not give any proofs though, since these results can be easily derived from the type $A$ specializations of the corresponding statements in Section 1; pointers to these statements are provided, wherever appropriate.

4.1. Bruhat cells and double Bruhat cells for $G L_{n}$. Our first object of interest are the double Bruhat cells. Let us introduce them for the group $G=G L_{n}(\mathbb{C})$. We will need some notation. Let $B$ (resp. $B_{-}$) be the subgroup of upper-triangular (resp. lower-triangular) matrices in $G$. Let $W=S_{n}$ be the symmetric group acting on the set $[1, n]=\{1, \ldots, n\}$; we will think of $W$ as a subgroup of $G$ by identifying a permutation $w$ with the matrix $w=\left(\delta_{i, w(j)}\right)$. The double cosets $B w B$ and $B_{-} w B_{-}$are called Bruhat cells (with respect to $B$ and $B_{-}$, respectively). The group $G$ has two Bruhat decompositions into a disjoint union of Bruhat cells (see, e.g., $[1$, Section 2.4]):

$$
G=\bigcup_{u \in W} B u B=\bigcup_{v \in W} B_{-} v B_{-} .
$$

The double Bruhat cells $G^{u, v}$ are defined by

$$
G^{u, v}=B u B \cap B_{-} v B_{-} ;
$$

thus $G$ is the disjoint union of all $G^{u, v}$ for $(u, v) \in W \times W$.

As an algebraic variety, a double Bruhat cell $G^{u, v}$ is biregularly isomorphic to a Zariski open subset of an affine space of dimension $n+\ell(u)+\ell(v)$, where $\ell(u)$ is the number of inversions of a permutation $u$; cf. Theorem 1.1. (In other words, $G^{u, v}$ is isomorphic, as an algebraic variety, to a subset of $\mathbb{C}^{n+\ell(u)+\ell(v)}$ obtained by excluding common zeroes of a finite set of polynomials.)

Each Bruhat cell (hence each double Bruhat cell) can be described explicitly by a set of conditions specifying vanishing and nonvanishing of certain minors. Let us denote by $\Delta_{I, J}$ the minor with the row set $I$ and the column set $J$; here $I$ and $J$ are two subsets of the same size in $[1, n]$, and the minor is viewed as a (regular) function on $G$. (This notation corresponds to that of Definition 1.4, as follows: the function $\Delta_{u \omega_{i}, v \omega_{i}}$ in (1.10) becomes the minor $\left.\Delta_{u([1, i]), v([1, i])}.\right)$ The following description of Bruhat cells is probably the most "economical".

Proposition 4.1. A matrix $x \in G$ belongs to the Bruhat cell $B w B$ if and only if it satisfies the following conditions:

- $\Delta_{w([1, i]),[1, i]} \neq 0$, for $i=1, \ldots, n-1$;

- $\Delta_{w([1, i-1] \cup\{j\}),[1, i]}=0$, for all $(i, j)$ such that $1 \leq i<j \leq n$ and $w(i)<w(j)$. 
This proposition can be proved by specializing Propositions 2.9 and 2.6 and Corollary 2.5. Notice that in our present situation the subgroup $N_{-}(w)$ (cf. (2.30)) consists of all unipotent lower-triangular matrices $y$ such that $y_{i j}=0$ whenever $w(i)>w(j)$.

The transpose map $x \mapsto x^{T}$ transforms a minor $\Delta_{I, J}$ into $\Delta_{J, I}$ and sends a Bruhat cell $B w B$ to $B_{-} w^{-1} B_{-}$. Thus Proposition 4.1 implies a similar description of the opposite Bruhat cells $B_{-} w B_{-}$. Combining the two sets of conditions yields an explicit description of the double Bruhat cells.

4.2. Factorization problem for $G L_{n}$. In the situation under consideration, the maximal torus $H=B \cap B_{-}$in $G$ is the subgroup of invertible diagonal matrices. Thus $H$ is naturally identified with $\mathbb{C}_{\neq 0}^{n}$ by taking the diagonal entries as coordinates. This allows us to state the factorization problem of Section 1.2 in a more symmetric form, as follows.

Let $E_{i, j}$ denote the $n \times n$ matrix whose $(i, j)$-entry is equal to 1 while all other entries are 0 ; let $I \in G$ denote the identity matrix. For $i=1, \ldots, n-1$, let

$$
x_{i}(t)=I+t E_{i, i+1}=\left(\begin{array}{cccccc}
1 & \cdots & 0 & 0 & \cdots & 0 \\
\cdots & \cdots & \cdots & \cdots & \cdots & \cdots \\
0 & \cdots & 1 & t & \cdots & 0 \\
0 & \cdots & 0 & 1 & \cdots & 0 \\
\cdots & \cdots & \cdots & \cdots & \cdots & \cdots \\
0 & \cdots & 0 & 0 & \cdots & 1
\end{array}\right)
$$

and

$$
x_{\bar{i}}(t)=I+t E_{i+1, i}=\left(\begin{array}{cccccc}
1 & \cdots & 0 & 0 & \cdots & 0 \\
\cdots & \cdots & \cdots & \cdots & \cdots & \cdots \\
0 & \cdots & 1 & 0 & \cdots & 0 \\
0 & \cdots & t & 1 & \cdots & 0 \\
\cdots & \cdots & \cdots & \cdots & \cdots & \cdots \\
0 & \cdots & 0 & 0 & \cdots & 1
\end{array}\right) .
$$

Also, for $i=1, \ldots, n$ and $t \neq 0$, let

$$
x_{(2)}(t)=I+(t-1) E_{i, i}=\left(\begin{array}{cccccc}
1 & \cdots & 0 & 0 & \cdots & 0 \\
\cdots & \cdots & \cdots & \cdots & \cdots & \cdots \\
0 & \cdots & t & 0 & \cdots & 0 \\
0 & \cdots & 0 & 1 & \cdots & 0 \\
\cdots & \cdots & \cdots & \cdots & \cdots & \cdots \\
0 & \cdots & 0 & 0 & \cdots & 1
\end{array}\right) .
$$

The matrices defined in (4.1)-(4.3) are called elementary Jacobi matrices. It is easy to see that these matrices generate $G$ as a group.

Consider the alphabet of $3 n-2$ symbols

$$
\mathcal{A}=\{1, \ldots, n-1, \text { (1) }, \ldots, \text { (n) }, \overline{1}, \ldots, \overline{n-1}\} \text {. }
$$

The formulas (4.1)-(4.3) associate a matrix $x_{i}(t) \in G$ to any symbol $i \in \mathcal{A}$ and any $t \in \mathbb{C}_{\neq 0}$. An analogue of the product map (1.3) is now defined as follows: to any sequence $\mathbf{i}=\left(i_{1}, \ldots, i_{l}\right)$ of symbols in $\mathcal{A}$, we associate the map $x_{\mathbf{i}}: \mathbb{C}_{\neq 0}^{l} \rightarrow G$ defined by

$$
x_{\mathbf{i}}\left(t_{1}, \ldots, t_{l}\right)=x_{i_{1}}\left(t_{1}\right) \cdots x_{i_{l}}\left(t_{l}\right)
$$


(Thus the difference with (1.3) is that now the factor $a \in H$ is split into elementary factors, which are allowed to be spread along the factorization.)

For instance, the sequence $\mathbf{i}=$ (1) $\overline{1}(2) 1$ gives rise to the map

$$
\left(t_{1}, t_{2}, t_{3}, t_{4}\right) \mapsto\left(\begin{array}{cc}
t_{1} & 0 \\
0 & 1
\end{array}\right)\left(\begin{array}{cc}
1 & 0 \\
t_{2} & 1
\end{array}\right)\left(\begin{array}{cc}
1 & 0 \\
0 & t_{3}
\end{array}\right)\left(\begin{array}{cc}
1 & t_{4} \\
0 & 1
\end{array}\right)=\left(\begin{array}{cc}
t_{1} & t_{1} t_{4} \\
t_{2} & t_{2} t_{4}+t_{3}
\end{array}\right)
$$

The matrix $x=x_{\mathbf{i}}\left(t_{1}, \ldots, t_{l}\right)$ has a simple combinatorial description in terms of planar networks. This description (cf. [6] and references therein) generalizes the one in [3, Section 2.4], and provides combinatorial formulas for the minors of $x$ as polynomials with nonnegative coefficients in the variables $t_{1}, \ldots, t_{l}$.

The planar network $\Gamma(\mathbf{i})$ associated to a sequence $\mathbf{i}$ of symbols from $\mathcal{A}$ (see Figure 1) is constructed as a concatenation of "elementary" networks that correspond to the parameters $t_{1}, \ldots, t_{l}$ (in this order). Each unbarred, barred, or circled entry $i_{k}$ of $\mathbf{i}$ corresponds to a fragment of one of the following three kinds, respectively:

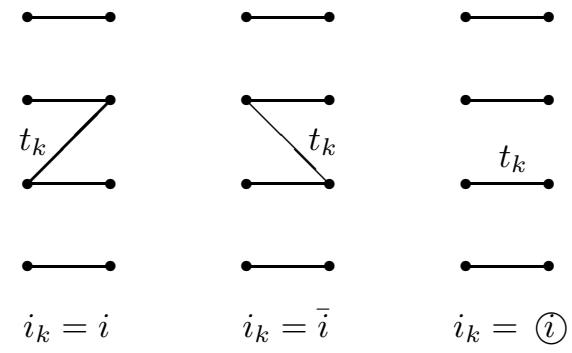

(a diagonal edge connects horizontal levels $i$ and $i+1$; in the examples above, $i=2$ ). These fragments are the combinatorial equivalents of the elementary matrices (4.1)(4.3). Each fragment has a distinguished edge whose weight is $t_{k}$; all other edges have weight 1 . All edges are presumed oriented left-to-right.

We number the sources and sinks of the network $\Gamma(\mathbf{i})$ bottom-to-top, and define the weight of a path in $\Gamma(\mathbf{i})$ to be the product of the weights of all edges in the path. One easily checks that the sum of these weights, over all paths that connect a given source $i$ to a given sink $j$, is nothing but the matrix element $x_{i j}$ of $x=x_{\mathbf{i}}\left(t_{1}, \ldots, t_{l}\right)$.

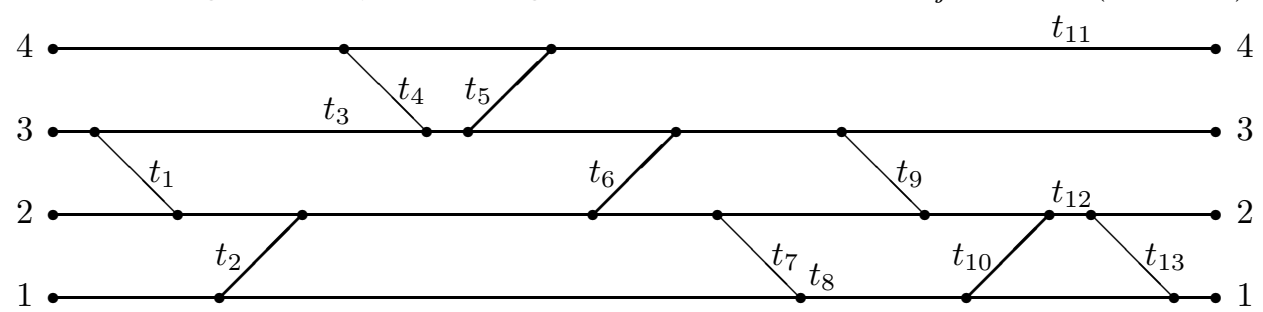

$\mathbf{i}=\overline{2} 1$ (3) $\overline{3} 32 \overline{1}$ (1) $\overline{2} 1$ (4) (2) $\overline{1}$

FiguRE 1. Planar network

This observation can be generalized. Let us define the weight of a family of paths in $\Gamma(\mathbf{i})$ to be the product of the weights of all paths in the family. Then the minors of $x$ are computed as follows.

Proposition 4.2. A minor $\Delta_{I, J}(x)$ equals the sum of weights of all families of vertex-disjoint paths in $\Gamma(\mathbf{i})$ connecting the sources labeled by I with the sinks labeled by $J$. 
For example, in Figure 1 we have

$$
x_{21}=t_{7} t_{8}+t_{12} t_{13}+t_{6} t_{9} t_{12} t_{13} \quad \text { and } \quad \Delta_{12,12}(x)=t_{8} t_{12}\left(1+t_{6} t_{9}\right) .
$$

We will be especially interested in a particular class of sequences $\mathbf{i}$ which we call factorization schemes (they are analogues of double reduced words of Section 1.2).

Definition 4.3. Let $u$ and $v$ be two permutations in $W=S_{n}$. A factorization scheme of type $(u, v)$ is a word $\mathbf{i}$ of length $n+\ell(u)+\ell(v)$ in the alphabet $\mathcal{A}$ which is an arbitrary shuffle of three words of the following kind:

- a reduced word for $v$;

- a reduced word for $u$, with all entries barred;

- a permutation of the symbols (1), .., (n).

These three words will be called, respectively, the E-part, the F-part, and the $H$-part of a factorization scheme $\mathbf{i}$.

For example, let

$$
\begin{aligned}
& u=4312=s_{2} s_{3} s_{1} s_{2} s_{1} \in S_{4}, \\
& v=4213=s_{1} s_{3} s_{2} s_{1} \in S_{4} .
\end{aligned}
$$

Then

$$
\mathbf{i}=\overline{2} 1 \text { (3) } \overline{3} 32 \overline{1} \text { (1) } \overline{2} 1 \text { (4) (2) } \overline{1}
$$

is a factorization scheme of type $(u, v)$.

The following result is an analogue of Theorem 1.2.

Theorem 4.4. Let $u, v \in W=S_{n}$, and let $l=n+\ell(u)+\ell(v)$. For any factorization scheme $\mathbf{i}$ of type $(u, v)$, the product map $x_{\mathbf{i}}$ given by (4.5) is a biregular isomorphism between $\mathbb{C}_{\neq 0}^{l}$ and a Zariski open subset of the double Bruhat cell $G^{u, v}$.

The factorization problem for $G L_{n}$ can now be formulated as follows: for a given factorization scheme $\mathbf{i}$, find explicit formulas for the components $t_{k}$ in terms of the matrix $x=x_{\mathbf{i}}\left(t_{1}, \ldots, t_{l}\right)$. By Theorem 4.4, each $t_{k}$ is a rational function in the matrix entries of $x$. For example, if $\mathbf{i}=$ (1) $\overline{1}$ (2) 1 , so that the map $x_{\mathbf{i}}$ is given by (4.6), then the solution to the factorization problem is given by

$$
t_{1}=x_{11}, t_{2}=x_{21}, t_{3}=\frac{\operatorname{det}(x)}{x_{11}}, t_{4}=\frac{x_{12}}{x_{11}} .
$$

4.3. The twist maps for $G L_{n}$. As in the general case, our solution to the factorization problem for $G=G L_{n}$ will utilize the "twist maps" $\zeta^{u, v}: x \mapsto x^{\prime}$, which are defined for any two permutations $u$ and $v$. The definition (1.12) can be rewritten as

$$
\left.x^{\prime}=d_{0}\left[x^{T} \bar{u}\right]_{+} \bar{u}^{T}\left(x^{T}\right)^{-1}{\overline{v^{-1}}}_{v^{-1}}^{T} x^{T}\right]_{-} d_{0}^{-1},
$$

where the following notation is used. The matrix $d_{0}$ is the diagonal $n \times n$ matrix with diagonal entries $1,-1,1,-1, \ldots$ For a matrix $z \in G, z^{T}$ stands for the transpose of $z$, and $z=[z]_{-}[z]_{0}[z]_{+}$denotes the Gaussian decomposition of $z$ (also known as the $L D U$ decomposition). Finally, the matrix $\bar{w}$ is obtained from a permutation matrix for $w$ by the following modification: an entry is changed from 1 to -1 whenever it has an odd number of nonzero entries lying below and to the left of it. 
By Theorem 1.6, the right-hand side of (4.10) is well defined for any $x \in G^{u, v}$, and the twist map $\zeta^{u, v}$ establishes a biregular isomorphism between $G^{u, v}$ and $G^{u^{-1}, v^{-1}}$; the inverse isomorphism is $\zeta^{u^{-1}, v^{-1}}$.

We give below a few examples of explicitly computed twist maps.

Example 4.5. Let $G=G L_{2}(\mathbb{C})$ and $u=v=w_{\mathrm{o}}$. Then (cf. Example 1.8)

$$
\begin{aligned}
& \bar{u}=\overline{v^{-1}}=\left(\begin{array}{cc}
0 & -1 \\
1 & 0
\end{array}\right)
\end{aligned}
$$

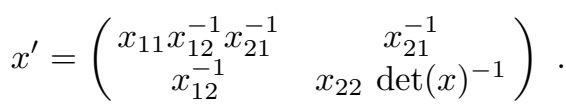

Example 4.6. Let $G=G L_{3}(\mathbb{C})$ and $u=v=w_{\mathrm{o}}$. Then

$$
x^{\prime}=\left(\begin{array}{ccc}
\frac{x_{11}}{x_{31} x_{13}} & \frac{\Delta_{12,13}}{x_{31} \Delta_{12,23}} & \frac{1}{x_{31}} \\
\frac{\Delta_{13,12}}{x_{13} \Delta_{23,12}} & \frac{x_{33} \Delta_{12,12}-\operatorname{det}(x)}{\Delta_{23,12} \Delta_{12,23}} & \frac{x_{32}}{\Delta_{23,12}} \\
\frac{1}{x_{13}} & \frac{x_{23}}{\Delta_{12,23}} & \frac{\Delta_{23,23}}{\operatorname{det}(x)}
\end{array}\right) .
$$

Example 4.7. Let $G=G L_{4}(\mathbb{C})$ and $u=v=w_{\mathrm{o}}$. Then $x^{\prime}$ is equal to

$$
\left(\begin{array}{cccc}
\frac{x_{11}}{x_{14} x_{41}} & \frac{\Delta_{12,14}}{x_{41} \Delta_{12,34}} & \frac{\Delta_{123,134}}{x_{41} \Delta_{123,234}} & \frac{1}{x_{41}} \\
\frac{\Delta_{14,12}}{x_{14} \Delta_{34,12}} & \frac{x_{44} \Delta_{12,12}-\Delta_{124,124}}{\Delta_{34,12} \Delta_{12,34}} & \frac{x_{42} \Delta_{123,134}-x_{41} \Delta_{123,234}}{\Delta_{34,12} \Delta_{123,234}} & \frac{x_{42}}{\Delta_{34,12}} \\
\frac{\Delta_{134,123}}{x_{14} \Delta_{234,123}} & \frac{x_{24} \Delta_{134,123}-x_{14} \Delta_{234,123}}{\Delta_{12,34} \Delta_{234,123}} & \frac{\Delta_{123,123} \Delta_{34,34}-x_{33} \operatorname{det}(x)}{\Delta_{123,234} \Delta_{234,123}} & \frac{\Delta_{34,23}}{\Delta_{234,123}} \\
\frac{1}{x_{14}} & \frac{x_{24}}{\Delta_{12,34}} & \frac{\Delta_{23,34}}{\Delta_{123,234}} & \frac{\Delta_{234,234}}{\operatorname{det}(x)}
\end{array}\right) .
$$

Example 4.8. Let $n=4, u=4312$, and $v=4213$ (cf. (4.7)). Then

$$
\bar{u}=\left(\begin{array}{rrrr}
0 & 0 & 1 & 0 \\
0 & 0 & 0 & 1 \\
0 & -1 & 0 & 0 \\
1 & 0 & 0 & 0
\end{array}\right) \quad, \quad \overline{v^{-1}}=\left(\begin{array}{rrrr}
0 & 0 & 0 & -1 \\
0 & -1 & 0 & 0 \\
1 & 0 & 0 & 0 \\
0 & 0 & 1 & 0
\end{array}\right)
$$


and

$$
x^{\prime}=\left(\begin{array}{cccc}
\frac{x_{11}}{x_{41} x_{13}} & \frac{\Delta_{12,13}}{x_{41} \Delta_{12,23}} & \frac{\Delta_{123,123}}{x_{41} \Delta_{123,234}} & \frac{1}{x_{41}} \\
\frac{\Delta_{14,12}}{\Delta_{34,12} x_{13}} & \frac{x_{43} \Delta_{12,12}-\Delta_{124,123}}{\Delta_{34,12} \Delta_{12,23}} & \frac{\Delta_{123,123} x_{42}}{\Delta_{34,12} \Delta_{123,234}} & \frac{x_{42}}{\Delta_{34,12}} \\
\frac{1}{x_{13}} & \frac{x_{23}}{\Delta_{12,23}} & \frac{\Delta_{23,23}}{\Delta_{123,234}} & \frac{\Delta_{23,23}}{\Delta_{123,123}} \\
0 & \frac{x_{13}}{\Delta_{12,23}} & \frac{\Delta_{13,23}}{\Delta_{123,234}} & \frac{\Delta_{134,234}}{\operatorname{det}(x)}
\end{array}\right) .
$$

Note that, in the course of computing the matrix elements of $x^{\prime}$ above, one has to take into account the relations

$$
x_{14}=0, \quad x_{24}=0, \quad \Delta_{234,123}=0
$$

satisfied by the matrix elements of $x \in G^{u, v}$. In particular, our computation of $x_{44}^{\prime}$ used (4.11) in conjunction with Gröbner bases techniques (see, e.g., [7]).

4.4. Double pseudoline arrangements. As an essential new ingredient in our solution to the factorization problem for $G L_{n}$, we will represent a factorization scheme $\mathbf{i}$ geometrically by the corresponding double pseudoline arrangement (or double wiring diagram). This arrangement is obtained by superimposing two arrangements naturally associated to the $E$ - and $F$-part of $\mathbf{i}$ (cf. [3]).

To be self-contained, let us recall the definition of a pseudoline arrangement associated to a reduced word. This is best done by an example. Consider $v=$ $4213 \in S_{4}$, together with the reduced decomposition $v=s_{1} s_{3} s_{2} s_{1}$ (cf. (4.7)). The corresponding pseudoline arrangement is given in Figure 2; to each entry $i$ of $\mathbf{i}$, we associate a crossing at the $i$ th level, counting from the bottom.

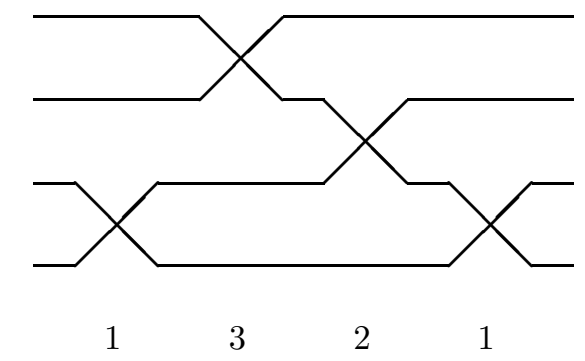

Figure 2. Pseudoline arrangement for the reduced word 1321

Let us now consider the factorization scheme $\mathbf{i}$ defined by (4.8). The $E$-part of $\mathbf{i}$ is 1321 , and we already drew the corresponding arrangement. The $F$-part of $\mathbf{i}$ is 23121. To construct the double pseudoline arrangement for $\mathbf{i}$, we superimpose the arrangements for 1321 and 23121, aligning them closely in the vertical direction, and placing the intersections so that tracing them left-to-right would produce the 
same shuffle of the two reduced words that appear in i. This results in the double pseudoline arrangement in Figure 3.

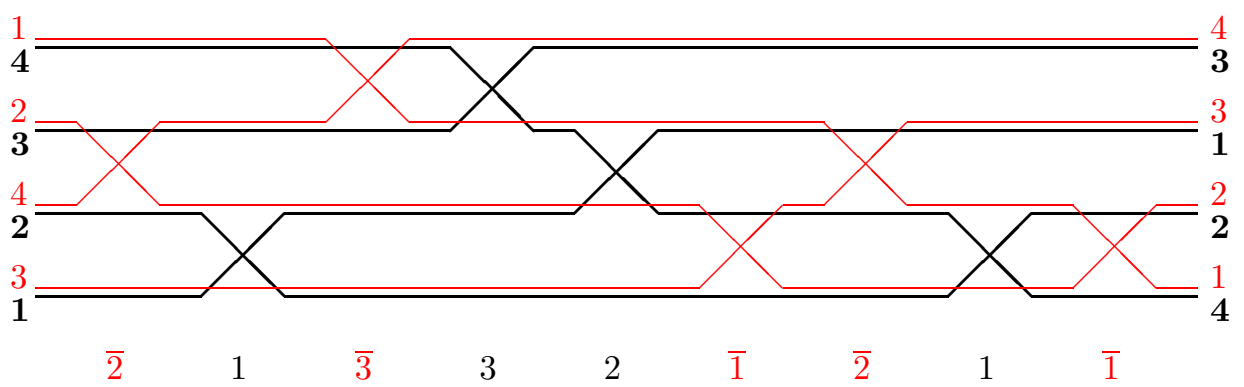

FiguRE 3. Double pseudoline arrangement

The double pseudoline arrangement that corresponds to a factorization scheme $\mathbf{i}$ is denoted by $\operatorname{Arr}(\mathbf{i})$. The two subarrangements of $\operatorname{Arr}(\mathbf{i})$ corresponding to the reduced words for $v$ and $u$ are called the $E$ - and $F$-part of $\operatorname{Arr}(\mathbf{i})$, and their crossing points are referred to as $E$ - and $F$-crossings, respectively. These crossings are in an obvious bijection with the noncircled entries of $\mathbf{i}$.

We next label the pseudolines of $\operatorname{Arr}(\mathbf{i})$ using the following important convention. The pseudolines of the $F$-part of $\operatorname{Arr}(\mathbf{i})$ are labeled 1 through $n$ bottom-up at the right end of the arrangement (just as in [3]). At the same time, the pseudolines of the $E$-part are labeled bottom-up at the left end. See Figure 3.

Another numbering that we are going to use is the bottom-to-top numbering of the $n-1$ horizontal strips containing the crossings of the arrangement. We say that the strip between the $j$ th and $(j+1)$ st horizontal lines, counting from the bottom, has level $j$, and all the $E$ - and $F$-crossings contained in this strip are of level $j$.

Note that arrangement $\operatorname{Arr}(\mathbf{i})$ does not depend on the $H$-part of the factorization scheme i. In order to include the $H$-part into the picture, we associate with each entry (1) a bullet $\bullet$ placed on the $j$ th horizontal line. The position of a bullet corresponds to the position of (1) in $\mathbf{i}$, so that when the arrangement is traced leftto-right, the crossings and bullets appear in the same order as the entries of $\mathbf{i}$ that they represent. The resulting "rigged" arrangement is denoted by Arr.(i). Figure 4 shows Arr.(i) for the factorization scheme (4.8).

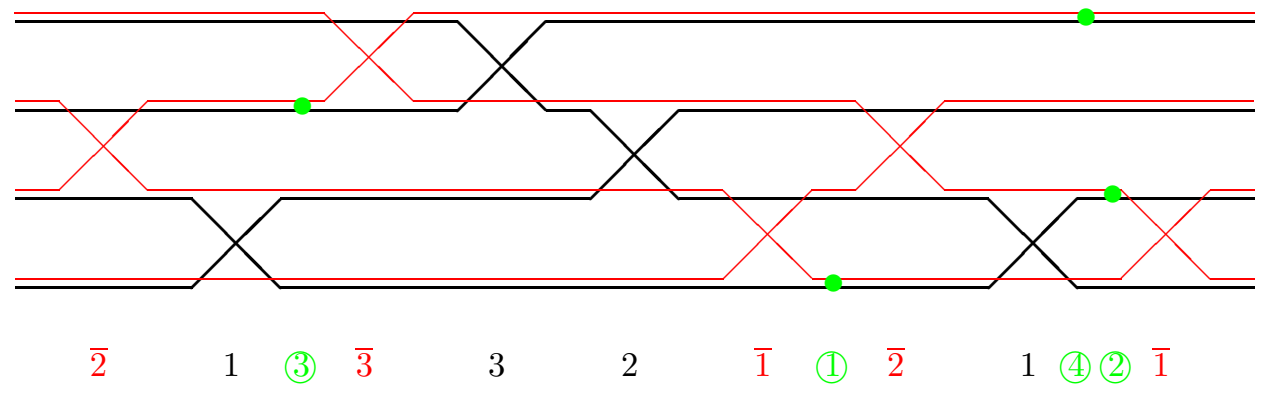

Figure 4. Arrangement Arr॰(i) 


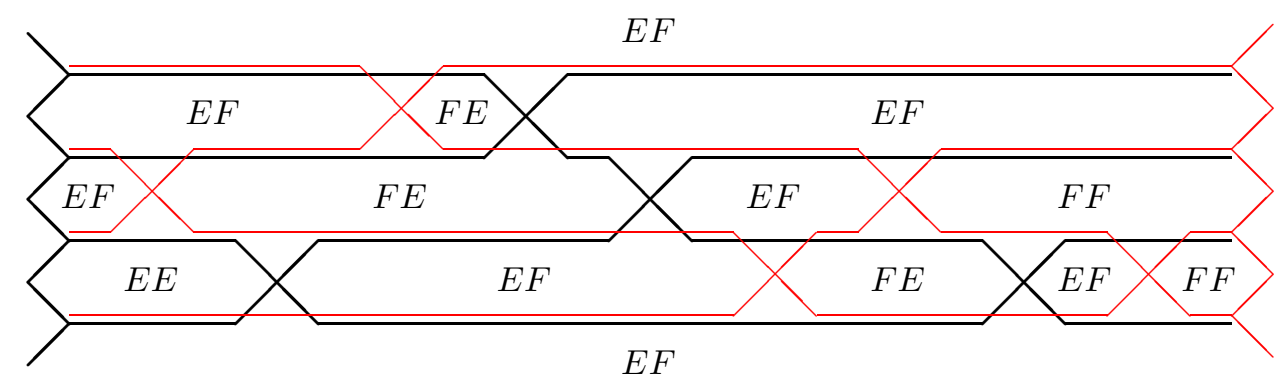

Figure 5. Types of chambers

To make our terminology uniform, we will refer to the bullets in Arr.(i) as $H$ crossings (despite the fact that they are not crossings geometrically). Thus the total number of crossings in $\operatorname{Arr}_{\bullet}(\mathbf{i})$ is $l=n+l(u)+l(v)$, and they are associated with the variables $t_{k}$ in the factorization (4.5). We will occasionally refer to the crossing in Arr.(i) associated with a factorization parameter $t_{k}$ by simply saying "crossing $t_{k}$ ". The $H$-crossing lying on the $i$ th horizontal line will also be denoted by $d_{i}$.

4.5. Solution to the factorization problem. Let us fix permutations $u, v \in S_{n}$ and a factorization scheme $\mathbf{i}$ of type $(u, v)$; in this section, we present our solution to the corresponding factorization problem. As in [3], the combinatorics needed to formulate the answer involves not only the crossings of the arrangement $\operatorname{Arr}(\mathbf{i})$ but also its chambers, which can be defined as horizontal segments between consecutive crossings of the same level. More precisely, each horizontal strip with, say, $k$ crossings breaks down into $k+1$ chambers (including the ones at the ends of the strip). Two more chambers are located at the bottom and the top of the arrangement. To illustrate, the arrangement in Figure 3 has 14 chambers; in general, there are $l+1$ of them.

We say that a chamber $C$ is of type $E F$ if the left endpoint of $C$ is an $E$ crossing, while its right endpoint is an $F$-crossing. Chambers of types $E E, F E$ and $F F$ are defined in a similar way. Figure 5 shows the types of all 14 chambers of the arrangement in Figure 3. Here and in the sequel, we use the following important convention: on each level, there is a fictitious $E$-crossing at the left border of the arrangement, and a fictitious $F$-crossing at the right border. These fictitious crossings determine the types of the chambers adjacent to the boundary of $\operatorname{Arr}(\mathbf{i})$.

For every chamber $C$ in $\operatorname{Arr}(\mathbf{i})$, let $I(C)$ denote the set of labels of the lines of the $F$-part of the arrangement that pass below $C$. Analogously, $J(C)$ will consist of the labels of lines of the $F$-part of $\operatorname{Arr}(\mathbf{i})$ that pass below $C$. The sets $I(C)$ and $J(C)$ are called chamber sets for the factorization scheme i. Figure 6 shows the chamber sets $I(C)$ and $J(C)$ for each chamber of the given arrangement. Note that if $C$ is a chamber of level $i$, then both $I(C)$ and $J(C)$ have $i$ elements.

Our constructions will also involve the "big" chambers formed by the $E$-part and the $F$-part of a double pseudoline arrangement, taken separately. We will refer to these "big" chambers as $E$-chambers and $F$-chambers, respectively. For example, the arrangement in Figure 3 has $9 E$-chambers, which are in obvious bijection with the 9 chambers in Figure 2. 


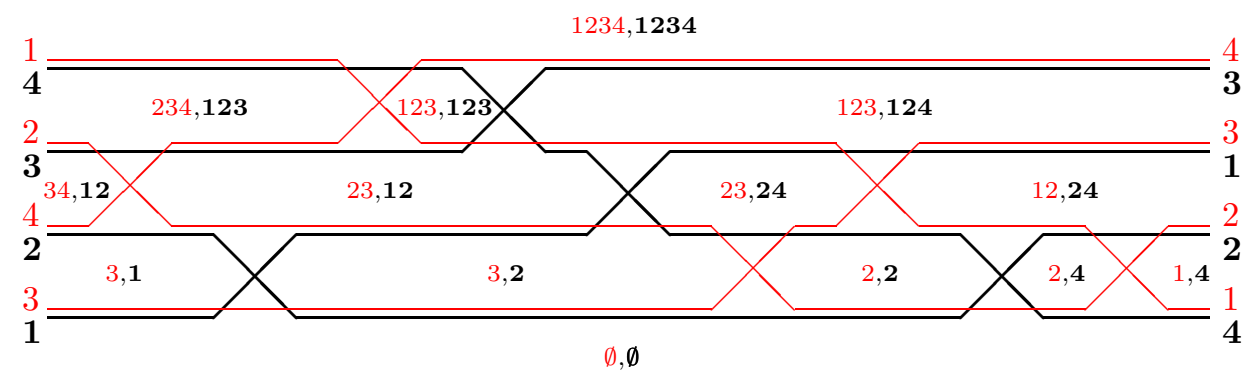

Figure 6. Chamber sets

For every chamber $C$ of the arrangement $\operatorname{Arr}(\mathbf{i})$, we denote

$$
M_{C}=\Delta_{I(C), J(C)}
$$

this minor is considered as a regular function on $G$ (with the convention that $\left.\Delta_{\emptyset, \emptyset}=1\right)$. For example, if $C$ is the rightmost chamber of level 2 in Figure 6, then $M_{C}=\Delta_{12,24}$.

To each $i=1, \ldots, n$ we associate a rational function on $G^{u, v}$ given by

$$
\Pi_{i}=\frac{\prod_{C} M_{C}}{\prod_{C^{\prime}} M_{C^{\prime}}}
$$

where $C$ runs over all chambers of level $i$ and type $F E$, while $C^{\prime}$ runs over all chambers of level $i$ and type $E F$. For example, in Figure 6 we have

$$
\Pi_{2}=\frac{\Delta_{23,12}}{\Delta_{34,12} \Delta_{23,24}} .
$$

Also, by convention, $\Pi_{0}=1$.

Let $\mathcal{C}$ be a "big" $K$-chamber of level $i$, where $K$ is one of the symbols $E$ and $F$. Let $L$ be the other of these symbols (i.e., $L=F$ if $K=E$, and $L=E$ if $K=F$ ). We define

$$
\mathcal{M}_{\mathcal{C}}^{\mathrm{right}}=\frac{\tilde{M} \prod_{C^{\prime}} M_{C^{\prime}}}{\prod_{C^{\prime \prime}} M_{C^{\prime \prime}}}
$$

where

- $C^{\prime}$ runs over all chambers of level $i$ and type $L K$ to the right of $\mathcal{C}$;

- $C^{\prime \prime}$ runs over all chambers of level $i$ and type $K L$ to the right of $\mathcal{C}$;

- $\tilde{M}=M_{C}$, where $C$ is the ("small") chamber at the right end of $\mathcal{C}$ (inside $\mathcal{C}$ ), unless $K=E$ and $C$ is stuck to the right border, in which case $\tilde{M}=1$.

Analogously,

$$
\mathcal{M}_{\mathcal{C}}^{\mathrm{left}}=\frac{\tilde{M} \prod_{C^{\prime}} M_{C^{\prime}}}{\prod_{C^{\prime \prime}} M_{C^{\prime \prime}}}
$$

where

- $C^{\prime}$ runs over all chambers of level $i$ and type $K L$ to the left of $\mathcal{C}$;

- $C^{\prime \prime}$ runs over all chambers of level $i$ and type $L K$ to the left of $\mathcal{C}$;

- $\tilde{M}=M_{C}$, where $C$ is the ("small") chamber at the left end of $\mathcal{C}$ (inside $\mathcal{C}$ ), unless $K=F$ and $C$ is stuck to the left border, in which case $\tilde{M}=1$.

We are finally prepared to state our solution to the factorization problem. 
Theorem 4.9. Let $\mathbf{i}$ be a factorization scheme of type $(u, v)$, and suppose a matrix $x \in G^{u, v}$ admits the factorization $x=x_{\mathbf{i}}\left(t_{1}, \ldots, t_{l}\right)$ with all $t_{k}$ nonzero complex numbers. Let $x^{\prime}=\zeta^{u, v}(x) \in G^{u^{-1}, v^{-1}}$ denote the matrix obtained from $x$ by the "twist" (4.10). Then the factorization parameters $t_{k}$ are determined as follows.

- If $t_{k}$ corresponds to the $H$-crossing $d_{i}$, then

$$
t_{k}=\frac{\Pi_{i}\left(x^{\prime}\right)}{\Pi_{i-1}\left(x^{\prime}\right)},
$$

where $\Pi_{i}$ and $\Pi_{i-1}$ are given by (4.12).

- Let $t_{k}$ correspond to an $E$ - or $F$-crossing of level $i$, and let $\mathcal{A}, \mathcal{B}, \mathcal{C}, \mathcal{D}$ be the four "big" chambers surrounding this crossing, as shown:

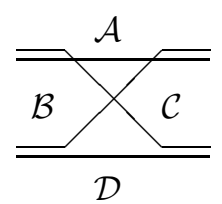

Then

$$
t_{k}=\frac{\mathcal{M}_{\mathcal{A}}^{\mathrm{opp}\left(d_{i+1}\right)}\left(x^{\prime}\right) \mathcal{M}_{\mathcal{D}}^{\mathrm{opp}\left(d_{i}\right)}\left(x^{\prime}\right)}{\mathcal{M}_{\mathcal{B}}^{\mathrm{opp}\left(d_{i+1}\right)}\left(x^{\prime}\right) \mathcal{M}_{\mathcal{C}}^{\mathrm{opp}\left(d_{i}\right)}\left(x^{\prime}\right)}
$$

where we refer to the notation of (4.13)-(4.14) as follows: the superscript $\operatorname{opp}\left(d_{i}\right)$ stands for "left" if the $H$-crossing $d_{i}$ is to the right of $t_{k}$, and for "right" if $d_{i}$ is to the left of $t_{k}$.

Theorem 4.9 is obtained as a specialization of Theorem 1.9, with the help of the following additional commutation relations:

$$
\begin{aligned}
& x_{i}(a) x_{(1)}(b)=x_{\text {(1) }}(b) x_{i}(a), j \notin\{i, i+1\} ; \\
& x_{i}(a) x_{(i)}(b)=x_{(i)}(b) x_{i}(a / b) ; \\
& x_{i}(a) x_{(1)}(b)=x_{(1)}(b) x_{i}(a b), j=i+1 ; \\
& x_{\bar{i}}(a) x_{(1)}(b)=x_{(1)}(b) x_{\bar{i}}(a), j \notin\{i, i+1\} ; \\
& x_{\bar{i}}(a) x_{(i)}(b)=x_{(i)}(b) x_{\bar{i}}(a b) ; \\
& x_{\bar{i}}(a) x_{(1)}(b)=x_{(1)}(b) x_{i}(a / b), j=i+1 .
\end{aligned}
$$

Example 4.10. To illustrate Theorem 4.9, let us compute the factorization parameter $t_{9}$ corresponding to the rightmost $F$-crossing of level 2 in Figure 4 . It is given by

$$
t_{9}=\frac{\mathcal{M}_{\mathcal{A}}^{\mathrm{right}}\left(x^{\prime}\right) \mathcal{M}_{\mathcal{D}}^{\mathrm{left}}\left(x^{\prime}\right)}{\mathcal{M}_{\mathcal{B}}^{\mathrm{right}}\left(x^{\prime}\right) \mathcal{M}_{\mathcal{C}}^{\mathrm{left}}\left(x^{\prime}\right)}
$$


where

$$
\begin{aligned}
& \mathcal{M}^{\text {right }}(\mathcal{A})=\Delta_{123,124}, \\
& \mathcal{M}^{\text {right }}(\mathcal{B})=\Delta_{23,24}, \mathcal{M}^{\text {left }}(\mathcal{C})=\frac{\Delta_{12,24} \Delta_{23,12}}{\Delta_{23,24} \Delta_{34,12}}, \\
& \mathcal{M}^{\text {left }}(\mathcal{D})=\frac{\Delta_{2,2}}{\Delta_{3,2}} .
\end{aligned}
$$

Hence

$$
t_{9}=\frac{\Delta_{2,2} \Delta_{34,12} \Delta_{123,124}}{\Delta_{3,2} \Delta_{12,24} \Delta_{23,12}}\left(x^{\prime}\right)
$$

Substituting the twisted matrix $x^{\prime}$ from Example 4.8 into (4.18) and simplifying, we finally obtain

$$
t_{9}=\frac{\Delta_{23,12}(x)\left(x_{43} \Delta_{12,12}(x)-\Delta_{124,123}(x)\right)}{x_{23} \Delta_{24,12}(x) \Delta_{123,123}(x)} .
$$

This answer can be verified directly using the combinatorial interpretation of minors $\Delta_{I, J}$ in terms of planar networks (see Proposition 4.2). From Figure 1 one obtains: $\Delta_{23,12}=t_{3} t_{7} t_{8} t_{9} t_{12}, x_{43}=t_{4}, \Delta_{12,12}=t_{8} t_{12}\left(1+t_{6} t_{9}\right), \Delta_{124,123}=t_{4} t_{8} t_{12}$, $x_{23}=t_{6}, \Delta_{24,12}=t_{4} t_{7} t_{8} t_{9} t_{12}, \Delta_{123,123}=t_{3} t_{8} t_{12}$, implying (4.19).

As in Theorem 1.10, formulas (4.15) and (4.16) imply that the factorization parameters $t_{1}, \ldots, t_{l}$ are related by an invertible monomial transformation to the $l$ minors $\Delta_{I(C), J(C)}\left(x^{\prime}\right)$ of the twisted matrix $x^{\prime}$ that correspond to the chambers of the arrangement $\operatorname{Arr}(\mathbf{i})$, with the bottom chamber excluded. The inverse transformation has the following description which can be deduced from (1.21) (since we left the latter formula without proof, the same is true for our next theorem, although it is not hard to give it a direct proof).

Theorem 4.11. Formulas (4.15)-(4.16) are equivalent to the following formulas:

$$
\Delta_{I(C), J(C)}\left(x^{\prime}\right)=\left(\prod t_{k}\right)^{-1}
$$

where the product is over all $t_{k}$ which correspond to the following types of crossings:

- E-crossings to the right of $C$ such that $C$ lies between the lines intersecting at $t_{k}$

- F-crossings to the left of $C$ such that $C$ lies between the lines intersecting at $t_{k}$;

- $H$-crossings to the right of $C$ such that $C$ lies above the E-line passing through $t_{k}$;

- $H$-crossings to the left of $C$ such that $C$ lies above the F-line passing through $t_{k}$.

For example, in Figure $6, \Delta_{3,1}\left(x^{\prime}\right)=\left(t_{2} t_{6}\right)^{-1}, \Delta_{123,124}\left(x^{\prime}\right)=\left(t_{1} t_{4} t_{8} t_{12}\right)^{-1}$, etc.

4.6. Applications to total positivity. In the case $G=G L_{n}(\mathbb{C})$, the definition of the totally nonnegative variety $G_{\geq 0}$ given in Section 1.3 is modified as follows: $G_{\geq 0}$ is the multiplicative semigroup generated by elementary Jacobi matrices (cf. (4.1)(4.3)) $x_{i}(t), x_{\bar{i}}(t)$, and $x_{(2)}(t)$ with $t>0$. It is known [17, 24] that this definition of total nonnegativity is equivalent to the classical one: an invertible matrix $x \in G$ belongs to $G_{\geq 0}$ if and only if all minors $\Delta_{I, J}(x)$ (in particular, all matrix entries) are nonnegative. 
For any two permutations $u$ and $v$ in $S_{n}$, the corresponding totally positive variety is defined by

$$
G_{>0}^{u, v}=G_{\geq 0} \cap G^{u, v} .
$$

Each factorization scheme of type $(u, v)$ gives rise to a parametrization of $G_{>0}^{u, v}$, according to the following analogue of Theorem 1.3.

Theorem 4.12. For any factorization scheme $\mathbf{i}=\left(i_{1}, \ldots, i_{l}\right)$ of type $(u, v)$, the corresponding product map $x_{\mathbf{i}}: \mathbb{C}_{\neq 0}^{l} \rightarrow G^{u, v}$ given by (4.5) restricts to a bijection between $\mathbb{R}_{>0}^{l}$ and $G_{>0}^{u, v}$.

The twist map $\zeta^{u, v}$ defined by (4.10) respects total positivity: it sends totally nonnegative matrices in $G^{u, v}$ to totally nonnegative matrices in $G^{u^{-1}, v^{-1}}$ (cf. Theorem 1.7). Combining this fact with Theorems 4.12 and 4.9 leads to a family of criteria for total positivity, one for each factorization scheme.

For a factorization scheme $\mathbf{i}=\left(i_{1}, \ldots, i_{l}\right)$ of type $(u, v)$, let $F(\mathbf{i})$ denote the collection of $l$ minors $\Delta_{u I(C), v^{-1} J(C)}$, where $C$ runs over all chambers of the arrangement $\operatorname{Arr}(\mathbf{i})$, excluding the bottom chamber. (This notation agrees with that of (1.22).) We note that the pair $\left(u I(C), v^{-1} J(C)\right)$ will correspond in the same way as above to a chamber $C$ if we relabel the pseudolines in $\operatorname{Arr}(\mathbf{i})$, numbering the $F$-pseudolines 1 through $n$ bottom-up at the left end, and the $E$-pseudolines bottom-up at the right end. See Figure 7.

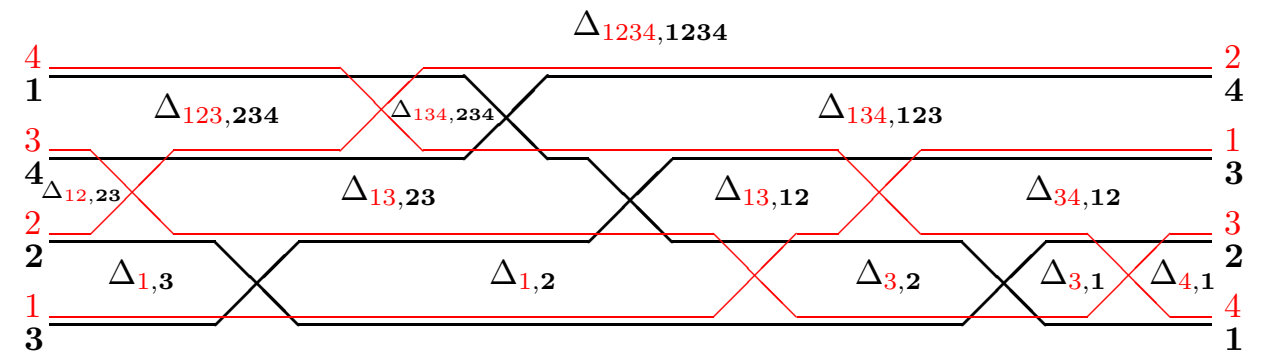

Figure 7. Minors $\Delta_{u I(C), v^{-1} J(C)}(x)$

Let $F(u, v)$ denote the union of the collections $F(\mathbf{i})$ for all factorization schemes i of type $(u, v)$. The set $F(u, v)$ can be described directly in the following way. A subset $I \subset[1, n]$ is called a $w$-chamber set if, together with each element $j$ it also contains every $i$ such that $i<j$ and $w(i)<w(j)$. In this terminology (originally introduced in [3, Section 5.3]), $F(u, v)$ consists of all minors $\Delta_{I, J}$ such that $I$ is a $u^{-1}$-chamber set while $J$ is a $v$-chamber set.

The following result specializes Theorem 1.11.

Theorem 4.13. Let $x$ be a matrix in a double Bruhat cell $G^{u, v}$, and let $\mathbf{i}$ be a factorization scheme of type $(u, v)$. Then the following are equivalent:

(1) $x$ is totally nonnegative;

(2) $\Delta_{I, J}(x)>0$ for any $u^{-1}$-chamber set $I$ and any $v$-chamber set $J$;

(3) $\Delta_{u I(C), v^{-1} J(C)}(x)>0$ for any chamber $C$ of the arrangement $\operatorname{Arr}(\mathbf{i})$.

For instance, in our running example where $u, v$, and $\mathbf{i}$ are given by (4.7) and (4.8), a matrix $x \in G^{u, v}$ is totally nonnegative if and only if the 13 minors appearing in Figure 7 are all positive if evaluated at $x$. 
Specializing Theorem 4.13 to the case $u=v=w_{\mathrm{o}}$, we see that the totally positive variety $G_{>0}^{w_{\mathrm{o}}, w_{\mathrm{o}}}$ is the classical variety $G_{>0}$ of the totally positive $n \times n$ matrices, i.e., those matrices whose all minors are (strictly) positive. Condition (3) of Theorem 4.13 provides a family of criteria for total positivity, each of which says that a matrix $x$ is totally positive if and only if some collection of $n^{2}$ minors are positive at $x$.

Different factorization schemes $\mathbf{i}$ and $\mathbf{i}^{\prime}$ of the same type $(u, v)$ can have the same collections of chamber sets, thus leading to the same criteria for total positivity. We will say that $\mathbf{i}$ and $\mathbf{i}^{\prime}$ (and the corresponding arrangements $\operatorname{Arr}(\mathbf{i})$ and $\operatorname{Arr}\left(\mathbf{i}^{\prime}\right)$ ) are isotopic if they can be obtained from each other by a sequence of the following "trivial 2-moves":

$$
\begin{aligned}
& \cdots i j \cdots \quad \sim \quad \cdots j i \cdots, \quad|i-j| \geq 2, \\
& \cdots \bar{i} \bar{j} \ldots \quad \sim \quad \cdots \bar{j} \bar{i} \ldots, \quad|i-j| \geq 2, \\
& \cdots i \bar{j} \cdots \quad \sim \quad \cdots \bar{j} i \cdots, \quad i \neq j, \\
& \cdots \text { (i) } j \cdots \sim \cdots j \text { (i) } \cdots \text {, } \\
& \cdots \text { (i) } \bar{j} \cdots \sim \cdots \bar{j}(\text { i) } \cdots \text {, } \\
& \cdots \text { (i) (1) } \cdots \sim \cdots \text { (1) (2) } \cdots \text {. }
\end{aligned}
$$

It is not hard to show that $\mathbf{i}$ and $\mathbf{i}^{\prime}$ have the same collection of chamber sets $(I(C), J(C))$ if and only if they are isotopic. Thus total positivity criteria in Theorem 4.13 are in a bijection with "isotopy types" of arrangements of type $(u, v)$.

The set of all isotopy types of arrangements of type $(u, v)$ has a natural structure of a graph defined as follows. We call two isotopy types adjacent if the corresponding collections of chamber sets are obtained from each other by exchanging a single pair $(I(C), J(C))$ with another one. The graph obtained this way is always connected, and its study is an interesting combinatorial problem. One can check that the adjacency relation in this graph corresponds to the following 3-moves and mixed 2-moves on double reduced words:

$$
\begin{array}{llll}
\cdots i j i \cdots & \rightsquigarrow & \cdots j i j \cdots, & |i-j|=1, \\
\cdots \bar{i} \bar{j} \bar{i} \cdots & \rightsquigarrow & \cdots \bar{j} \bar{i} \bar{j} \cdots, & |i-j|=1, \\
\cdots i \bar{i} \cdots & \rightsquigarrow & \cdots \bar{i} i \cdots
\end{array}
$$

(cf. Sections 3.3 and 3.4); the connectedness property follows from Proposition 3.7.

For $G=G L_{2}$ and $u=v=w_{\mathrm{o}}$, there are 2 isotopy types. The corresponding collections $F(\mathbf{i})$ are $\left\{x_{11}, x_{12}, x_{21}, \operatorname{det}(x)\right\}$ and $\left\{x_{22}, x_{12}, x_{21}\right.$, $\left.\operatorname{det}(x)\right\}$.

In the case of $G L_{3}$ and $u=v=w_{\mathrm{o}}$, there are 34 isotopy types, giving rise to 34 different total positivity criteria. Each of these criteria involves 9 minors. Five of them-the minors

$$
x_{31}, x_{13}, \Delta_{23,12}, \Delta_{12,23}, \operatorname{det}(x)
$$


- are common to all 34 criteria; they correspond to the "unbounded" chambers lying on the periphery of each arrangement. The other four minors that distinguish isotopy types from each other correspond to the bounded chambers. Figure 8 shows a graph with 34 vertices labeled by the quadruples of "bounded" minors that appear in the corresponding total positivity criteria.

For an arbitrary $n$ (and $u=v=w_{\mathrm{o}}$ ), one obtains various nice (and surprising) total positivity criteria in $G L_{n}$ by making particular choices of (the isotopy type of) a double pseudoline arrangement in Theorem 4.13. Let us discuss two criteria obtained in this way.

A minor $\Delta_{I, J}$ is called solid if both $I$ and $J$ consist of several consecutive indices. A criterion due to Fekete [10] (see also [12, p. 299]) asserts that (strict) total positivity of a matrix is equivalent to the positivity of all its solid minors. Each of the two criteria described below will strengthen this result.

We will consider two factorization schemes of type $\left(w_{\mathrm{o}}, w_{\mathrm{o}}\right)$ having the same $E$ and $F$-parts (albeit shuffled in a different way). For both of them, the $E$-part is the lexicographically minimal reduced word for $w_{\mathrm{o}}$, i.e., the reduced word

$$
1,2,1,3,2,1, \ldots, n-1, n-2, \ldots, 1 ;
$$

the $F$-part is the same but with barred entries. Let $\mathbf{i}_{1}$ denote the shuffle of these parts such that all the unbarred entries precede the barred ones. Let $\mathbf{i}_{2}$ denote the shuffle of the same parts such that every unbarred entry is immediately followed by the corresponding barred entry (so that $\mathbf{i}_{2}$ starts with $1, \overline{1}, 2, \overline{2}, \ldots$ ). A direct check shows that the corresponding collections of minors $F\left(\mathbf{i}_{1}\right)$ and $F\left(\mathbf{i}_{2}\right)$ are given as follows:

- $F\left(\mathbf{i}_{1}\right)$ consists of solid minors $\Delta_{I, J}$ such that $1 \in I \cup J$;

- $F\left(\mathbf{i}_{2}\right)$ consists of solid minors $\Delta_{I, J}$ such that $\min (I)+\max (J) \in\{n, n+1\}$.

Each of these two collections consists of $n^{2}$ minors; and by Theorem 4.13, each of them provides a total positivity criterion that strengthens the one of Fekete's: a square matrix is totally positive if and only if all the minors in $F\left(\mathbf{i}_{1}\right)$ (respectively, $\left.F\left(\mathbf{i}_{2}\right)\right)$ are positive. It should be mentioned that the first of these criteria was (implicitly) obtained by Cryer [8, Theorems 1.1 and 3.1] using a result of Karlin [16, p. 85]; an explicit statement appears in [13, Theorem 4.1]. The second criterion seems to be new.

The equivalence of conditions (2) and (3) in Theorem 4.13 has the following algebraic explanation (cf. Theorem 1.12).

Theorem 4.14. For any factorization scheme $\mathbf{i}$ of type $(u, v)$, the collection of minors $F(\mathbf{i})$ is a totally positive base (cf. Definition 2.21) for the collection $F(u, v)$.

The most significant part of this theorem is that every minor from $F(u, v)$ can be written as a subtraction-free expression in the minors from $F(\mathbf{i})$. Such an expression can be found in a constructive way. To do this, it will be enough to consider two arrangements $\operatorname{Arr}(\mathbf{i})$ and $\operatorname{Arr}\left(\mathbf{i}^{\prime}\right)$ whose isotopy types are adjacent in the graph that we described above; recall that this means that the collection of minors $F\left(\mathbf{i}^{\prime}\right)$ is obtained from $F(\mathbf{i})$ by exchanging a single minor $\Delta$ with another minor $\Delta^{\prime}$. It suffices to show that $\Delta^{\prime}$ can be written as a subtraction-free expression in the minors from $F(\mathbf{i})$. This can be done with the help of certain 3-term determinantal identities. These identities are stated in the following proposition, which is a specialization of Theorems 1.16 and 1.17. We use the notation $L i, L i j$, etc., as a shorthand for $L \cup\{i\}, L \cup\{i, j\}$, etc. 


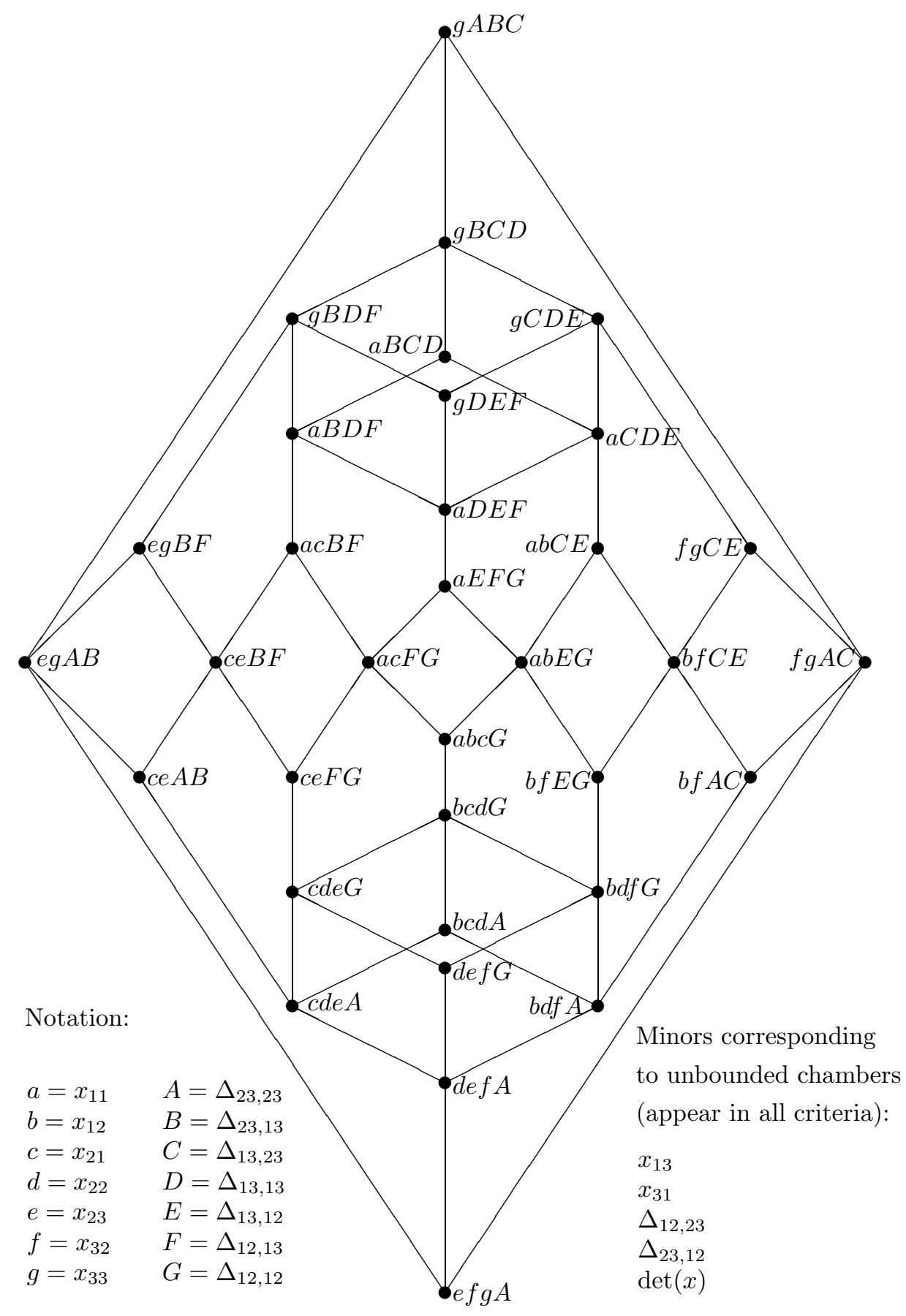

FIgURE 8. Total positivity criteria for $G L_{3}$ 
Proposition 4.15. For any $i, j, k, p \in[1, n]$ and $I, L \subset[1, n]$ such that $i<j<k$, $|I|=|L|+1, L \cap\{i, j, k\}=\emptyset, I \cap\{p\}=\emptyset$, we have:

$$
\begin{aligned}
& \Delta_{I p, L i k} \Delta_{I, L j}=\Delta_{I p, L i j} \Delta_{I, L k}+\Delta_{I p, L j k} \Delta_{I, L i}, \\
& \Delta_{L i k, I p} \Delta_{L j, I}=\Delta_{L i j, I p} \Delta_{L k, I}+\Delta_{L j k, I p} \Delta_{L i, I} .
\end{aligned}
$$

For any $i, i^{\prime}, j, j^{\prime} \in\{1, \ldots, n\}$ and $I, J \subset\{1, \ldots, n\}$ such that $i<i^{\prime}, j<j^{\prime}$, $|I|=|J|, I \cap\left\{i, i^{\prime}\right\}=J \cap\left\{j, j^{\prime}\right\}=\emptyset$, we have

$$
\Delta_{I i, J j} \Delta_{I i^{\prime}, J j^{\prime}}=\Delta_{I i, J j^{\prime}} \Delta_{I i^{\prime}, J j}+\Delta_{I, J} \Delta_{I i i^{\prime}, J j j^{\prime}} .
$$

The identities (4.23)-(4.24) are well known, although their attribution is complicated. As early as in 1819 they were proved by P. Desnanot (see [20, pp. 140142]). Identities (4.23) are special cases of the (Grassmann-)Plücker relations (see, e.g., [11, (15.53)]), while identity (4.24) plays a crucial role in C. L. Dodgson's condensation method, and because of that is occasionally associated with the name of Lewis Carroll.

It would be interesting to see which other classical determinantal identities can be generalized to the functions $\Delta_{u \omega_{i}, v \omega_{i}}$ on any semisimple group. We conclude the paper by mentioning one challenging problem of this kind: find a generalization of the classical Binet-Cauchy formula for the minors of the product of two matrices:

$$
\Delta_{I, J}(x y)=\sum_{K} \Delta_{I, K}(x) \Delta_{K, J}(y) .
$$

At present, we only know such a generalization for the minuscule fundamental weights $\omega_{i}$.

Acknowledgments. Part of this paper was written when the authors were participating in the special program "Combinatorics" at MSRI in Berkeley in Spring 1997. The second author (A.Z.) gratefully acknowledges the hospitality of his colleagues in Buenos Aires (Alicia Dickenstein and Fernando Cukierman) and Strasbourg (Peter Littelmann and Olivier Mathieu), where he worked on parts of this paper; these visits were supported by the University of Buenos Aires and CNRS, France. A large part of our computations in Section 4 were performed with Maple.

\section{REFERENCES}

[1] J. L. Alperin and R. B. Bell, Groups and representations, Springer-Verlag, 1995. MR 96m:20001

[2] T. Ando, Totally positive matrices, Linear Algebra Appl. 90 (1987), 165-219. MR 88b:15023

[3] A. Berenstein, S. Fomin, and A. Zelevinsky, Parametrizations of canonical bases and totally positive matrices, Adv. Math. 122 (1996), 49-149. MR 98j:17008

[4] A. Berenstein and A. Zelevinsky, Total positivity in Schubert varieties, Comment. Math. Helv. 72 (1997), 128-166. CMP 97:14

[5] N. Bourbaki, Groupes et algèbres de Lie, Ch. IV-VI, Hermann, Paris, 1968. MR 39:1590

[6] F. Brenti, Combinatorics and total positivity, J. Combin. Theory, Ser. A 71 (1995), 175-218. MR 96f:05019

[7] D. Cox, J. Little, and D. O'Shea, Ideals, varieties, and algorithms, Springer-Verlag, 1996. MR 97h:13024

[8] C. Cryer, The $L U$-factorization of totally positive matrices, Linear Algebra Appl. 7 (1973), 83-92. MR 47:250

[9] V. V. Deodhar, On some geometric aspects of Bruhat orderings. I. A finer decomposition of Bruhat cells. Invent. Math. 79 (1985), 499-511. MR 86f:20045

[10] M. Fekete, Über ein Problem von Laguerre, Rendiconti del Circ. Mat. Palermo 34 (1912), 89-100, 110-120. 
[11] W. Fulton and J. Harris, Representation theory, Springer-Verlag, New York, 1991. MR 93a:20069

[12] F. R. Gantmacher and M. G. Krein, Oszillationsmatrizen, Oszillationskerne und Kleine Schwingungen Mechanischer Systeme, Akademie-Verlag, Berlin, 1960. (Russian original edition: Moscow-Leningrad, 1950.) MR 22:5161

[13] M. Gasca and J. M. Peña, Total positivity and Neville elimination, Linear Algebra Appl. 165 (1992), 25-44. MR 93d:15031

[14] A. Grothendieck, EGA IV, Publ. Math. IHES 32, 1967. MR 39:220

[15] J. E. Humphreys, Reflection groups and Coxeter groups, Cambridge University Press, 1990. MR 92h:20002

[16] S. Karlin, Total positivity, Stanford University Press, 1968. MR 37:5667

[17] C. Loewner, On totally positive matrices, Math. Z. 63 (1955), 338-340. MR 17:466f

[18] G. Lusztig, Total positivity in reductive groups, in: Lie theory and geometry: in honor of Bertram Kostant, Progress in Mathematics 123, Birkhäuser, 1994. MR 96m:20071

[19] G. Lusztig, Introduction to quantum groups, Progress in Mathematics 110, Birkhäuser, 1993. MR 94m: 17016

[20] T. Muir, The theory of determinants, 2nd edition, vol. 1, Macmillan, London, 1906.

[21] K. Rietsch, Intersections of Bruhat cells in real flag varieties, Intern. Math. Res. Notices 1997, no. 13, 623-640. MR 98f:14038

[22] B. Shapiro, M. Shapiro, and A. Vainshtein, Connected components in the intersection of two open opposite Schubert cells in $S L_{n}(\mathbb{R}) / B$, Intern. Math. Res. Notices 1997, no. 10, 469-493. MR 98e: 14054

[23] T. A. Springer, Linear algebraic groups, Progress in Mathematics 9, Birkhäuser, 1981. MR 84i:20002

[24] A. M. Whitney, A reduction theorem for totally positive matrices, J. d'Analyse Math. 2 (1952), 88-92. MR 14:732c

Department of Mathematics, Massachusetts Institute of Technology, Cambridge, MassachusetTS 02139

E-mail address: fomin@math.mit.edu

Department of Mathematics, Northeastern University, Boston, Massachusetts 02115

E-mail address: andrei@neu.edu 